\title{
Towards the development of redundancy assessment protocols for steel truss bridges
}

Jennifer M. Stains

West Virginia University

Follow this and additional works at: https://researchrepository.wvu.edu/etd

\section{Recommended Citation}

Stains, Jennifer M., "Towards the development of redundancy assessment protocols for steel truss bridges" (2012). Graduate Theses, Dissertations, and Problem Reports. 4923.

https://researchrepository.wvu.edu/etd/4923

This Thesis is protected by copyright and/or related rights. It has been brought to you by the The Research Repository @ WVU with permission from the rights-holder(s). You are free to use this Thesis in any way that is permitted by the copyright and related rights legislation that applies to your use. For other uses you must obtain permission from the rights-holder(s) directly, unless additional rights are indicated by a Creative Commons license in the record and/ or on the work itself. This Thesis has been accepted for inclusion in WVU Graduate Theses, Dissertations, and Problem Reports collection by an authorized administrator of The Research Repository @ WVU. For more information, please contact researchrepository@mail.wvu.edu. 


\title{
TOWARDS THE DEVELOPMENT OF REDUNDANCY ASSESSMENT Protocols For STEEL TruSs BRIDGeS
}

\author{
Jennifer M. Stains \\ Thesis submitted to the \\ Benjamin M. Statler College of Engineering and Mineral Resources \\ at West Virginia University \\ in partial fulfillment of the requirements \\ for the degree of \\ Master of Science \\ in \\ Civil and Environmental Engineering
}

Karl E. Barth, Ph.D., Chair

Udaya B. Halabe, Ph.D.

John P. Zaniewski, Ph.D.

Department of Civil and Environmental Engineering

Morgantown, West Virginia

2012

Keywords: redundancy, steel bridge, LRFD, finite element modeling, field testing 


\begin{abstract}
Towards The Development Of Redundancy Assessment Protocols For STEEL TRUSS BRIDGES
\end{abstract}

\author{
Jennifer M. Stains
}

On December 15, 1967, the Silver Bridge across the Ohio River catastrophically collapsed, resulting in the deaths of 46 people. Following the collapse of the Silver Bridge, one of the goals of the development of new bridge inspection and design guidelines was to create a more uniform level of safety in the U.S. highway infrastructure by standardizing inspection frequencies and qualifications for bridges. It was believed that regular maintenance procedures assured that these types of bridge systems possessed acceptable levels of safety. However, on August 1, 2007, the I-35-W Bridge in Minneapolis, MN, collapsed, resulting in 13 fatalities and 145 injuries. Its failure, like that of the Silver Bridge, was the result of failure of a single structural element.

Many engineers and researchers have classified these bridges as nonredundant, as failure of a single member/element caused failure, or in these cases, collapse, of the entire bridge. These types of structural failures have led the structural research community in an effort to quantify redundancy in highway bridge superstructures. Throughout the years, many researchers have done studies on levels of redundancy in highway bridges and presented methods (both deterministic- and probabilistically-based) for quantifying bridge redundancy. However, there is no method which is universally accepted; this is apparent when reviewing the current edition of the AASHTO LRFD Bridge Design Specifications (2010), which still incorporates an individual member design approach and does not address the concept of structural redundancy in any direct manner. In addition, in the bridge community there is disagreement as to what the actual definition of structural redundancy should be and what failure criteria should be considered.

The goal of this project is to develop appropriate protocols for assessing redundancy in steel truss highway bridges. To accomplish this, an extensive literature review was performed, focused on reviewing past studies on structural redundancy in highway bridges. Once performed, a set of redundancy protocols were developed, incorporating modern finite element modeling techniques and limit states associated with system capacity under damaged conditions. To validate the proposed modeling technique, a field test was performed on a representative truss bridge in Jackson County, WV. Once validated with experimental data, these protocols were then exercised on the representative truss to determine the effect of damaged conditions on system capacity of steel truss bridges. 


\section{ACKNOWLEDGMENTS}

I would like to thank my advisor, Dr. Karl Barth, for giving me the opportunity and guidance to pursue my Master's degree. I would also like to thank U.S. Bridge Corporation for funding this research and providing valuable feedback throughout this project.

I would also like to thank the graduate students of B-11 as they have always been there to provide technical feedback and support, as well as friendship. Additionally, I would like to thank my family and friends for always supporting me. Specifically, I would like to thank my parents, Keith and Donna Stains, as well as my sisters, Natalie and Meredith Stains, for their love and support, especially through my Master's work at WVU. I would not be who or where I am today without them. 


\section{TABLE OF CONTENTS}

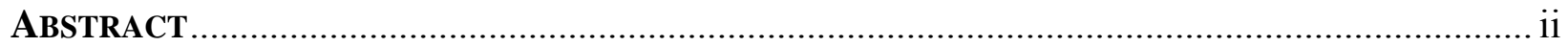

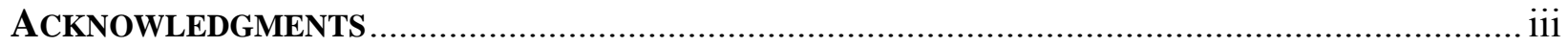

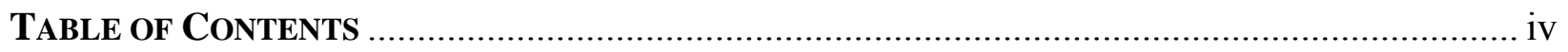

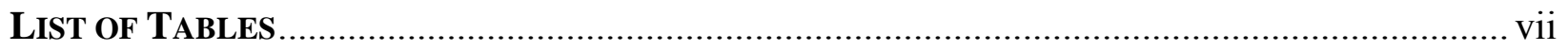

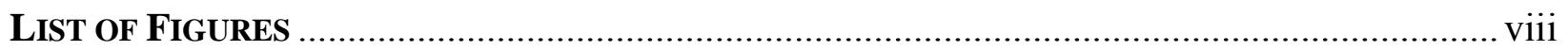

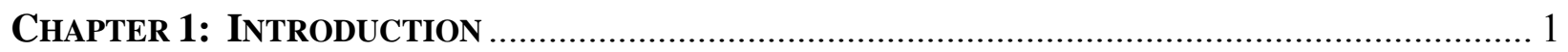

1.1 BACKGROUND / OVERVIEW ............................................................................................................... 1

1.2 PROJECT SCOPE \& OBJECTIVES.........................................................................................................2

1.3 THESIS ORGANIZATION_...................................................................................................................................

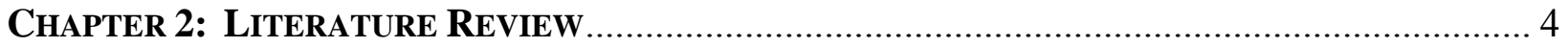

2.1 INTRODUCTION

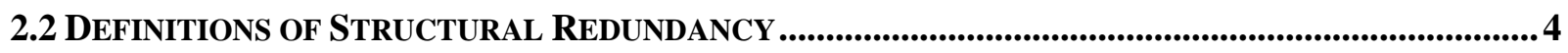

2.2.1 AASHTO LRFD Provisions for Redundancy ..................................................................... 5

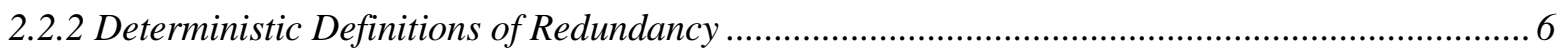

2.2.2.1 Generalized Definitions of Deterministic Redundancy ................................................... 7

2.2.2.2 Specific Definitions of Deterministic Redundancy ........................................................ 8

2.2.3 Probabilistic Definitions of Redundancy …............................................................................. 10

2.3 DEFINITIONS OF RELEVANT LIMIT STATES AND FAILURE CRITERIA ............................................11

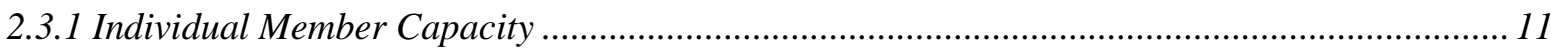

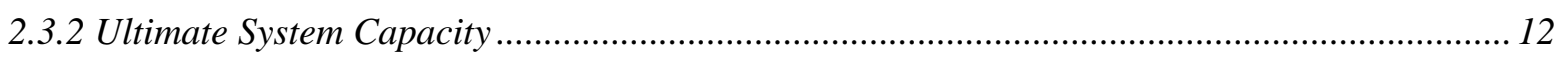

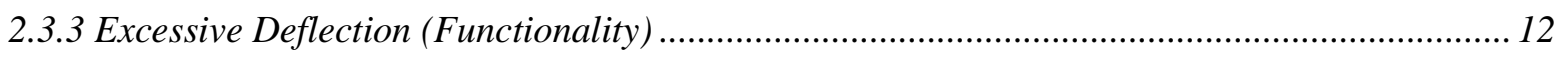

2.3.4 System Capacity under Damaged Conditions....................................................................... 13

2.4 FINDINGS OF PREVIOUS REDUNDANCY STUDIES ...................................................................................13

2.4.1 Bridge Superstructures with Two Parallel I-Girders............................................................. 14

2.4.2 Bridge Superstructures with Multiple Parallel I-Girders ....................................................... 16

2.4.3 Bridge Superstructures with Parallel Box Girders ................................................................... 20

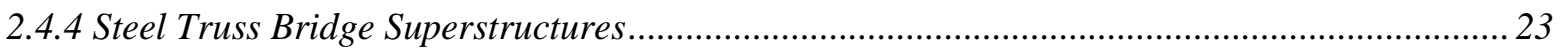


2.5 METHODS FOR QUANTIFYING REDUNDANCY IN HIGHWAY BRIDGE SUPERSTRUCTURES .........28

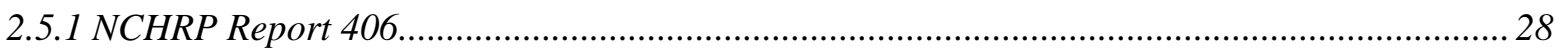

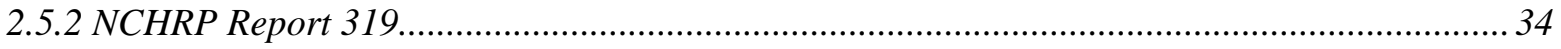

2.5.3 Additional Approaches for Redundancy Evaluation .............................................................. 36

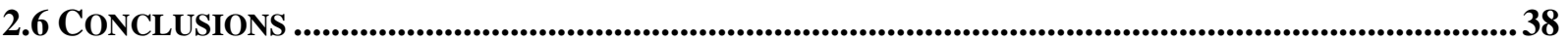

Chapter 3: Development of Protocols for the Accurate Assessment of Primary

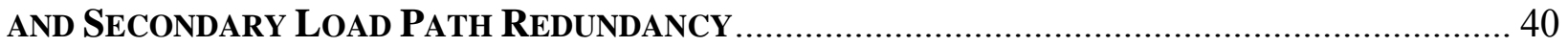

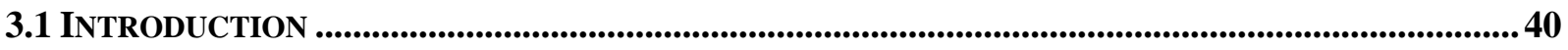

3.2 FINITE ELEMENT MODELING TECHNIQUES ...................................................................................40

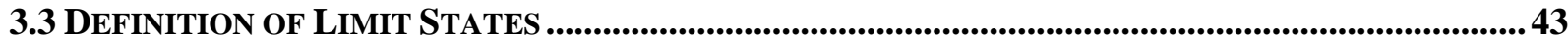

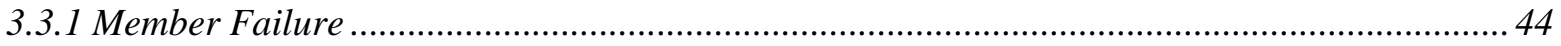

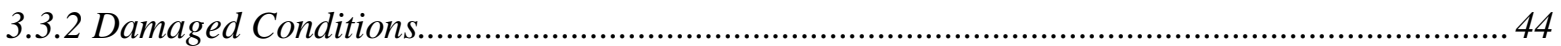

3.4 PROPOSED PRoCEdRE FOR REdUNDANCY EVALUATION .....................................................44

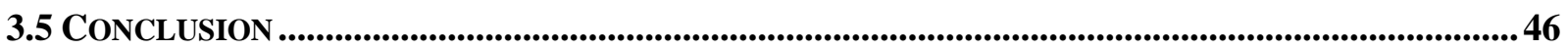

Chapter 4: Little Mill Creek BRidge field TeSt ................................................. 47

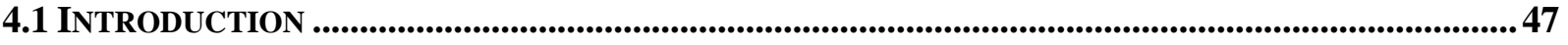

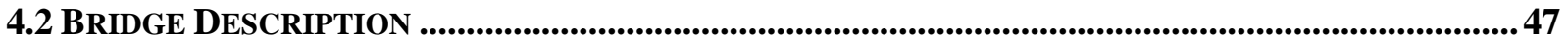

4.3 SUMMARY OF EQUIPMENT USED ...........................................................................................................48

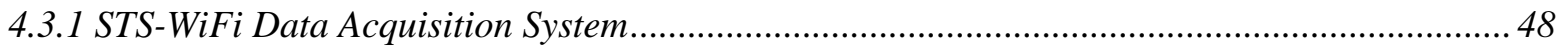

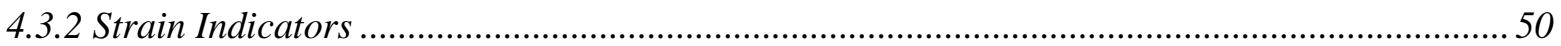

4.3.3 Linear Variable Differential Transducers (LVDTs) ................................................................. 50

4.3.4 WVDOH Load Truck \& Wheel Scales .................................................................................... 51

4.3.5 Miscellaneous Equipment …............................................................................................... 53

4.4 INSTRUMENTATION PLAN ..............................................................................................................................54

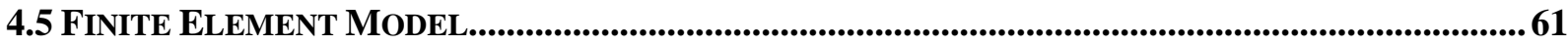

4.6 RESULTS AND COMPARISON WITH FINITE ELEMENT MODELING ................................................63

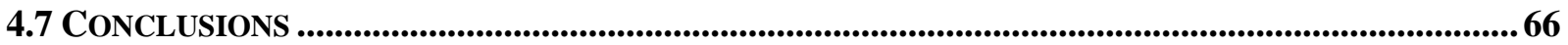

Chapter 5: Redundancy Assessment of Little Mill Creek Bridge ......................... 67

5.1 INTRODUCTION

5.2 SELECTED MEMBERS FOR REDUNDANCY EVALUATION_.....................................................667

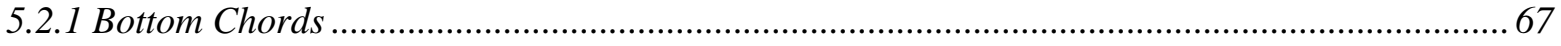

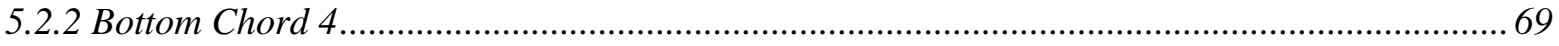




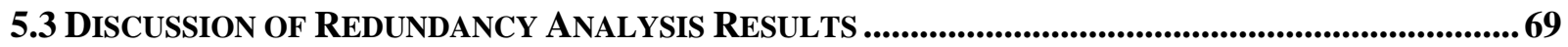

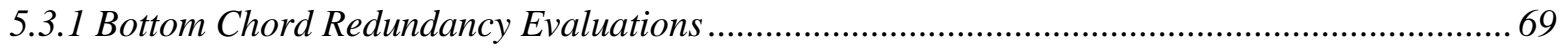

5.3.2 Bottom Chord 4: Internal Member Redundancy Evaluation .................................................. 70

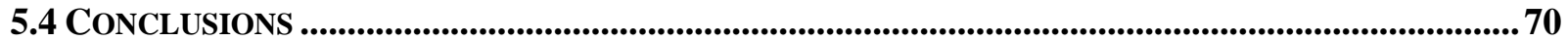

CHAPTER 6: SummaRY AND CONCLUding REMARKS ................................................... 72

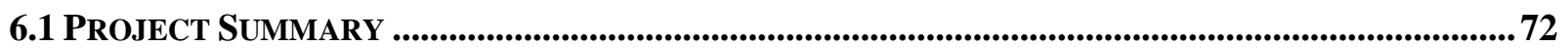

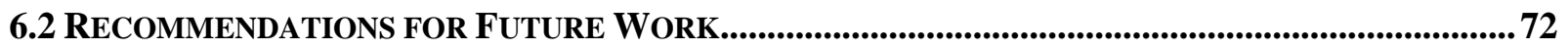

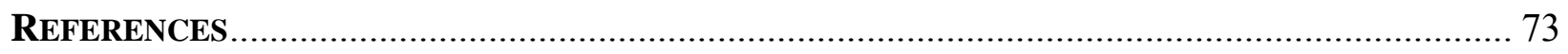

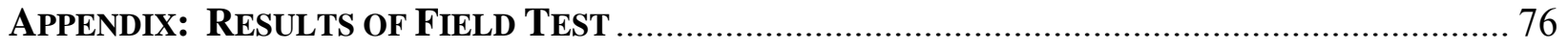

A.1 DESCRIPTION OF FIELD TEST DATA.........................................................................................................76

A.2 RUN 1 (SETUP \#1, EDGE TRUCK RUN) ...................................................................................................... 78

A.3 RUN 2 (SETUP \#2, CENTER TRUCK RUN) ............................................................................................8 87

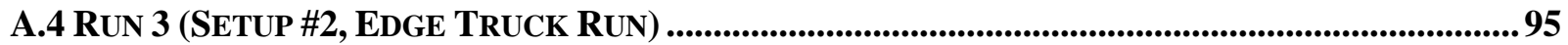




\section{LIST OF TABLES}

Table 2.1: Two-Girder Redundancy Results (Heins \& Kato, 1982)....................................... 15

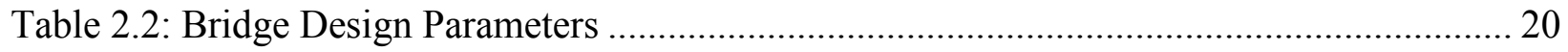

Table 2.3: System Factors for $60 \mathrm{ft}$ Span......................................................................... 21

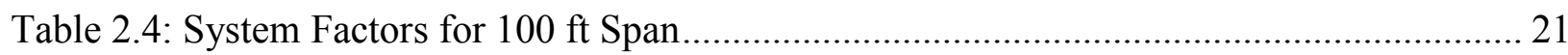

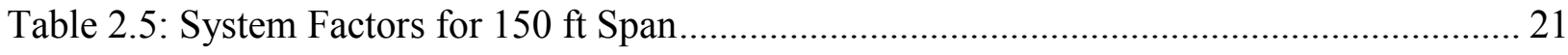

Table 2.6: Effects of Posttensioning on Redundancy Factors ............................................... 26

Table 5.1: Bottom Chord Redundancy Analysis Results .................................................... 70

Table 5.2: Bottom Chord 4 Redundancy Analysis Results ................................................... 70 


\section{LIST OF FIGURES}

Figure 2.1: NCHRP Report 406 Results for Simple Span, Six Steel I-Girder Layouts ............... 18

Figure 2.2: NCHRP Report 406 Results for Continuous Span, Six Steel I-Girder Layouts........ 19

Figure 2.3: NCHRP Report 406 Results for Simple Span, Steel Box Girder Layouts................. 22

Figure 2.4: Concrete Girders Used to Simulated HS-20 Live Loading (Hovell, 2007) .............. 23

Figure 2.5: Ambridge-Aliquippa Bridge (Holth and McOmber, 2006)..................................... 24

Figure 2.6: Post-tensioned tendon layouts (Ayyub and Ibrahim, 1990).................................. 25

Figure 2.7: Fracture-critical members in U.S. Bridge Series 3000 (BEST, 2000) ................... 28

Figure 3.1: Steel Stress-Strain Relationship (Galindez, 2009) ................................................ 41

Figure 3.2: Multi-linear Stress-Strain Approximations (Galindez, 2009) ............................... 42

Figure 3.3: AASHTO HL-93 Design Truck (AASHTO, 2010) ................................................ 43

Figure 4.1: Isometric View of Little Mill Creek Bridge ......................................................... 48

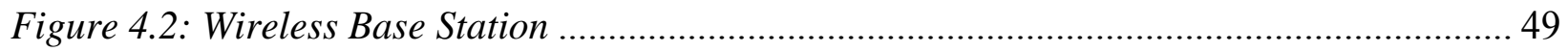

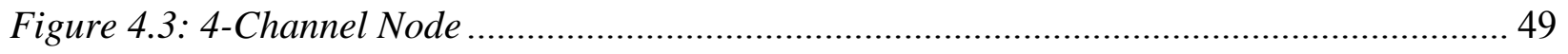

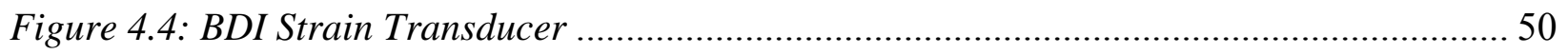

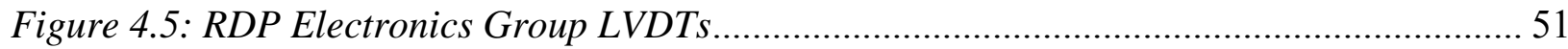

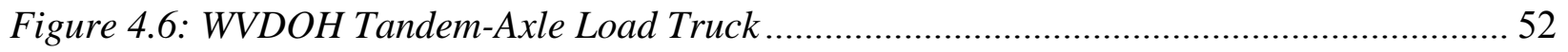

Figure 4.7: WV-DOH Tandem-Axle Truck Wheel Loads ..................................................... 52

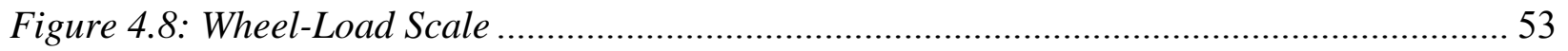

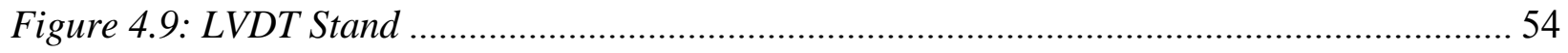

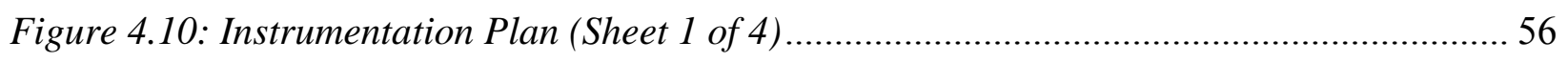

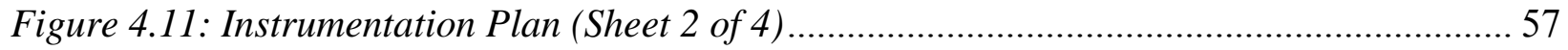

Figure 4.12: Instrumentation Plan (Sheet 3 of 4) .................................................................... 58

Figure 4.13: Instrumentation Plan (Sheet 4 of 4) ................................................................. 59

Figure 4.14: Load Truck Over Panel Point during Field Test ............................................. 61

Figure 4.15: Intact Finite Element Model Screen Capture ................................................... 62

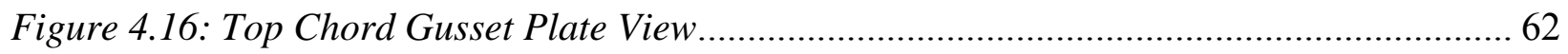

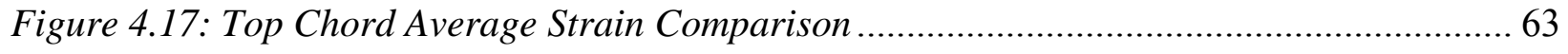

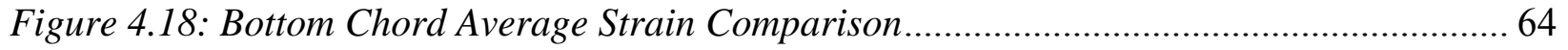

Figure 4.19: Diagonal Chord Average Strain Comparison ................................................... 64 


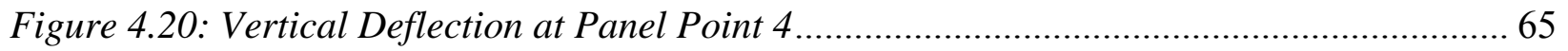

Figure 4.21: Vertical Deflection of Floor Beam 3 ................................................................ 65

Figure 5.1: Bottom Chords 1-5 Longitudinal Truck Placements ........................................... 68

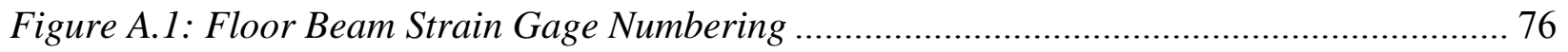

Figure A.2: Diagonal Strain Gage Numbering ..................................................................... 77

Figure A.3: Bottom Chord Strain Gage Numbering ............................................................ 77

Figure A.4: Top Chord Strain Gage Numbering .............................................................. 78 


\section{CHAPTER 1: INTRODUCTION}

\subsection{BACKGROUND / OVERVIEW}

On December 15, 1967, the U.S. Highway 35 bridge across the Ohio River (more commonly known as the Silver Bridge for its aluminum painting), a steel eyebar suspension / truss bridge spanning from Point Pleasant, WV to Kanagua, $\mathrm{OH}$, catastrophically collapsed, resulting in the deaths of 46 people. The collapse was the result of a single eyebar failing due to corrosion cracking and stress concentration, which, in turn, led to failure of the system. Following the collapse of the Silver Bridge, the 1968 Federal-Aid Highway Act and 1978 Surface Transportation Assistance Act led to the establishment of the National Bridge Inspection Standards (NBIS) (Agrawal et al, 2010). One of the goals of the development of these inspection guidelines was to create a more uniform level of safety in the U.S. highway infrastructure by standardizing inspection frequencies and qualifications for bridges. Over the next few years, steel truss bridges had earned the reputation of being economical and reliable (Liao et al., 2011). It was believed that maintenance procedures, like those prescribed in the NBIS, assured that these types of bridge systems possessed acceptable levels of safety. However, on August 1, 2007, the I-35-W interstate highway bridge, a steel deck truss over the Mississippi River in Minneapolis, MN, collapsed, resulting in 13 fatalities and 145 injuries. Its failure, like that of the Silver Bridge, was the result of failure of a single structural element; in this case, the critical component was a gusset plate which failed due to insufficient capacity.

Many engineers and researchers have classified these bridges as nonredundant, as failure of a single member/element caused failure, or in these cases, collapse, of the entire bridge. These types of structural failures have led the structural research community in an effort to quantify redundancy in highway bridge superstructures. Many researchers, bridge engineers, and owner agencies have expressed a growing concern over the degree of redundancy present in bridge systems.

Throughout the years, many researchers have done studies on levels of redundancy in highway bridges and presented methods (both deterministic- and probabilistically-based) for quantifying bridge redundancy. However, there is no method which is universally accepted; this 
is apparent when reviewing the current edition of the AASHTO LRFD Bridge Design Specifications (2010), which still incorporates an individual member design approach and does not address the concept of structural redundancy in any direct manner. In addition, in the bridge community there is disagreement as to what the actual definition of structural redundancy should be and what failure criteria should be considered.

\subsection{Project Scope \& ObJectives}

The focus of this project is to develop a set of protocols for assessing system redundancy in steel truss highway bridges. Specifically, this is accomplished in the following manner.

- A literature review focused on reviewing past studies on structural redundancy in highway bridges. Particular attention was paid to NCHRP Report 406 (Ghosn and Moses, 1997), the report whose work is regarded as one of the most comprehensive studies available on redundancy.

- A set of redundancy protocols along with appropriate limit states were developed to evaluate redundancy in steel truss highway bridges. These were based upon the limit states and protocols set forth in NCHRP Report 406. In addition, a refined finite element (F.E.) modeling technique is proposed.

- A physical load test was then performed on an existing steel truss bridge representative of the type of bridge focused on in this study. Strains and displacements collected from field data were compared with F.E. results to assess the validity of the modeling technique used in this effort.

- Finally, a redundancy assessment was performed on the bridge on which the load test was performed. Attention was paid to the built-up bottom chord members of the bridge, identified as fracture-critical by the WVDOH. 


\subsection{ThESIS ORgaNizATION}

A brief overview of the organization of this thesis is as follows:

- $\quad$ Chapter 2:

○ This chapter summarizes previous redundancy research, highlighting various applicable limit states, modeling techniques, methods used by various researchers for assessing redundancy, and previous investigations, on several types of bridge configurations.

- Chapter 3:

○ This chapter outlines the proposed protocols for determining the level of redundancy present in steel truss bridges. In addition, the finite element modeling technique used in this study is presented.

- Chapter 4:

- This chapter describes the physical load test which was performed on an representative steel truss bridge in Jackson Co., WV. In addition, the comparison of the load test data with the finite element model data is shown.

- Chapter 5:

- This chapter describes the redundancy analyses performed on the bottom chords of the representative steel truss bridge which was the subject of Chapter 4 .

- Chapter 6:

- This chapter provides a summary of the scope of work conducted for this study and highlights the key findings. Lastly, this chapter provides suggestions for future efforts in this area. 


\section{CHAPTER 2: LITERATURE REVIEW}

\subsection{INTRODUCTION}

The purpose of this chapter is to discuss previous research efforts related to evaluating levels of redundancy in bridge systems. A better understanding of structural redundancy could lead to a more efficient means of designing and rating highway bridges, thereby preventing disasters of the magnitudes described in Section 1.1. Presented in this chapter is a comprehensive review of previous studies focused on the concept of structural redundancy in highway bridges. Particular attention is paid to the definition of redundancy used by various researchers, the pertinent limit states associated with redundancy, specific findings in redundancy studies and research projects, and descriptions of the methods used to quantify redundancy in bridge systems.

\subsection{DEFINITIONS OF STRUCTURAL REDUNDANCY}

Throughout the years, numerous researchers have investigated redundancy in a variety of bridge systems and subsystems. However, there has never been a statement (either mathematical or descriptive) defining redundancy that has been universally accepted and/or adopted by the structural community. A unified and agreed-upon statement defining redundancy is a crucial first step in outlining a methodology to assess levels of redundancy in highway bridge systems. However, a comprehensive review of redundancy definitions in previous studies must be completed in order to develop an acceptable universal definition of structural redundancy. Presented in this section is an overview of the varying definitions of member and system redundancy found in previous research, both from deterministic and probability-based points-ofview. 


\subsubsection{AASHTO LRFD Provisions for Redundancy}

In addition to developing a more uniform level of safety in the design of highway bridge infrastructure, one of the key objectives of developing the AASHTO LRFD Specifications (as opposed to the former AASHTO Standard Specifications) was to enhance both the ductility and redundancy of the nation's bridges (AASHTO, 2010) (NSBA, 2010). To accomplish this, the following was adopted as the standard LRFD limit state expression.

$\sum \eta_{i} \gamma_{i} Q_{i} \leq \phi R_{n}$

Equation 2-1

Where: $\eta_{i}=$ load modifier relating to ductility, redundancy, and operational importance

$\gamma_{i}=$ load factor

$Q_{i}=$ force effect

$\phi=$ resistance factor

$R_{n}=$ nominal capacity

Specifically, the " $\eta_{i}$ " load modifier is a function of three separate quantities:

- $\quad \eta_{D}=$ factor relating to ductility

- $\eta_{I}=$ factor relating to operational importance

- $\eta_{R}=$ factor relating to redundancy

At the time of the development of the LRFD Specifications, calibrated system factors for redundancy, ductility, and operational importance had not yet been established. The " $\eta_{i}$ " factors present in the limit state expression were therefore installed as placeholders, leaving room for future development and calibration. In addition, an interesting point to note about the LRFD limit state expression is the location of these factors. For instance, while ductility and redundancy are physical attributes associated with structural resistance, they instead modify the loads applied. However, this placement is arbitrary; as stated in the commentary, improved quantification of these values can possibly lead to rearrangement of the limit state expression. 
Section 1.3.4 of the AASHTO LRFD Specifications is the only section dealing explicitly with redundancy. Its sole provision states that "multiple load-path and continuous structures should be used unless that there are compelling reasons not to use them." It also gives suggested bounds for " $\eta_{R}$ " values, specifically ranging from a minimum value of 0.95 (corresponding to exceptional levels of redundancy) to a maximum value of 1.05 (for nonredundant members). However, the commentary also states that this $\pm 5 \%$ range was selected subjectively in the absence of more precise information.

Finally, it is important to identify the lack of a concise definition of the term "redundancy" in this section of the Specifications. Each chapter of the AASHTO LRFD Specifications contains a "Definitions" section, which includes basic definitions of various terms and concepts. It is important to note that, while the "Definitions" section in Chapter 1 contains terms such as "ductility," "redundancy" is not defined. However, there is a somewhat nondescriptive definition in Chapter 6, which states that redundancy is "the quality of a bridge that enables it to perform its design function in a damaged state."

The lack of a formal definition of "redundancy" in the AASHTO LRFD Specifications directly implies that the bridge engineering community has not yet established what parameters are critical in evaluating redundancy in highway bridges. Therefore, a review of previous work in the field of bridge redundancy is necessary in order to better formulate a more suitable definition of redundancy as well as to develop the means to quantify it.

\subsubsection{Deterministic Definitions of Redundancy}

Redundancy, from an analytical and deterministic point of view, is a somewhat loosely defined term. However, regardless of the specific definition, many common trends exist in various researchers' points-of-view on the subject. Discussed in this section is a summary of the

various deterministic definitions of structural redundancy, with emphasis on highlighting commonalities among the findings. 


\subsubsection{Generalized Definitions of Deterministic Redundancy}

The concept of structural redundancy and its importance has been recognized by various bridge engineers and researchers for decades. However, even today, the specific definition remains under debate (Kanno and Ben-Haim, 2011). Many generalized statements have been proposed that involve system reserve capacity, formation of collapse mechanisms or plastic hinges, etc. However, no universal statement has been agreed upon.

On the other hand, there are some generalized definitions that many researchers have agreed upon. One such definition essentially states that redundancy is "the amount of reserve capacity that a system has after the failure or damage of one of its components." It should be noted that, while slight variations of this statement were found, the general meaning of this definition has held true in various research in the area of bridge system redundancy. Ghosn and Moses' (1997) state that bridge redundancy "consists of the capability of a bridge superstructure to carry loads after the damage or the failure of one of its members."

One of the important features of bridge systems that many researchers have indicated to directly impact redundancy is the presence/absence of fracture-critical members. AASHTO Specifications (2010) define fracture-critical members as "components in tension whose failure is expected to result in the collapse of the bridge or the inability of the bridge to perform its function." Identification and classification of fracture-critical members in highway bridges has been a topic of significant interest in the bridge engineering community since the failure of such bridges as the Silver Bridge and the I-35 Minneapolis Bridge (see Section 2.1). In 1978, AASHTO published the Guide Specifications for Fracture Critical Non-Redundant Steel Bridge Members. Specifically, the Guide outlines the responsibilities of designers, proper construction and welding procedures, and nondestructive testing procedures for evaluating and inspecting fracture-critical members. It should be noted that the definition of fracture-critical members in the Guide Specifications for Fracture Critical Non-Redundant Steel Bridge Members is carried forward in the current edition of the AASHTO LRFD Specifications.

Like structural redundancy, there are differing opinions among the bridge community about what factors should be considered in classifying a member as fracture-critical (Connor et al., 2005). For instance, the definitions of redundancy are not clear regarding the level of failure that would constitute a fracture-critical member. An example of this would be a bolted or riveted 
built-up member, where it is likely that a fracture would be limited to only one tension element because it could not propagate directly into neighboring elements. On the other hand, an extreme event, such as a catastrophic natural phenomenon, vehicle and boat crashes, or terrorist attacks, could destroy the entire member or render it unfit for use. Still, the failure of a single element of a highway bridge and its impact on the system is arguably the most discussed topic regarding a bridge's inherent redundancy. Many of the research efforts in the area of structural redundancy (which are discussed in Section 2.4) and the methods for quantifying redundancy in highway bridges (discussed in Section 2.5) are associated with the damage and/or removal of a given bridge element and determining the resulting capacity of the altered system.

Another critical phenomenon associated with generalized definitions of redundancy is collapse. AASHTO Specifications define collapse as a "major change in the geometry of a bridge that renders it unfit for use." Many of the definitions of structural redundancy are interrelated with the collapse of a bridge system. An example of this would be the definition outlined in NCHRP Report 406 (Ghosn and Moses, 1997). While redundancy is defined in this report as the capacity of a bridge after the failure of one of its members, that capacity is found by determining the maximum load that the structure can withstand before collapse occurs. For more details regarding this method of quantifying redundancy, see Section 2.5.1.

\subsubsection{Specific Definitions of Deterministic Redundancy}

While many researchers give broad definitions of redundancy, some have specifically categorized the various possible levels that may exist in highway bridge systems. One specific class of redundancy, termed load-path redundancy, essentially states that a system can develop a means of redistributing loads that does not exceed acceptable levels of stresses, deflections, etc. in the presence of failure/damage of a member/component. This alternate distribution of loads in the system is generally referred to as a load path. An example of load-path redundancy is a parallel girder bridge, where failure of one or more girders causes redistribution of loads to the remaining girders. This definition is reiterated in NCHRP 319 (Daniels et al., 1988), which states that "if the alternate load path is also capable of safely resisting the specified after-fracture dead and live loads and is further capable of maintaining after-fracture serviceability of the deck, it is called a redundant alternate load path." 
Another type of redundancy, termed structural redundancy, states that the failure of a member/component merely changes the boundary conditions or support conditions, but does not result in structural collapse. The NSBA Steel Bridge Handbook chapter on Redundancy (2010) cites an example of this being a two-span steel girder bridge. Since the moments in a two-span bridge are highest at the internal support, the bridge may fail in negative bending and possibly form a plastic hinge at the interior support. This, however, may not result in structural collapse if the positive bending region of the girders can withstand the bending moment resulting from the internal redistribution of loads.

One common link that many engineers make with redundancy is that statically indeterminate structures, by default, possess a certain degree of redundancy. (This association has also been reiterated by various researchers.) Ayyub and Ibrahim (1990) state that "statically indeterminate trusses have a higher level of redundancy than statically determinate ones." In addition, Frangopol and Curley (1987) defines various methods for quantifying structural redundancy (both deterministic and probabilistic), one of which simply being the degree of statical indeterminacy.

However, it should be noted that statical indeterminacy does not explicitly guarantee alternate viable load paths in any generic structure. While statically indeterminate structures do offer alternate loads paths in the event of damage to a structural component, this does not guarantee that these alternate load paths are sufficient to resist the applied loads, thereby preventing collapse. NCHRP 354 (Connor et al., 2005) differentiates these two concepts in their definition of redundancy, which states that a redundant system must provide alternate load paths. This definition is elaborated by stating that even structures with alternate load paths may be considered nonredundant if the members along the alternate load path do not have sufficient capacity to withstand the redistrubuted loads. This report goes on to state that statically determinate structures can be classified as redundant systems if alternative viable load paths can be developed in the presence of the failure/damage of a given member/component.

Finally, another localized type of redundancy, called internal or member redundancy, states that a member in question may be considered redundant if sufficient load paths exist within the member itself. Essentially, all of the individual components of a given member or component would have to reach failure for the entire member to fail. Purvis (1988) elaborates on this concept for steel members by stating that internal redundancy is related to the propagation of 
cracks through a member's cross-section. Examples of this type of redundancy may include systems of multiple rebar in a reinforced concrete beam (Nowak and Collins, 2000), multiples of a riveted steel plate or member (NSBA, 2010), and built-up steel sections. On the other hand, since rolled members are comprised of one solid component, they would lack internal redundancy on a member level.

\subsubsection{Probabilistic Definitions of Redundancy}

In a probabilistic sense, redundancy is generally defined by researchers in quantitative terms that relate to system reliability through the use of probabilities of failure. Specifically, these expressions typically employ the probability of failure of an entire system under damaged conditions and the probabilities of failure of individual damaged components in the system. Frangopol and Curley (1987) reiterate this by stating that system-level reliability methods should be used to examine the behavior of a damaged structure beyond the failure of a single element.

In addition, $\mathrm{Fu}$ and Frangopol (1998) define system redundancy as a safety margin, which is the capacity to prevent failure of an entire structural system upon failure of any component. This is expressed in terms of a redundancy index, RI, which is a function of both the

probability of damage/failure of a single structural element and the probability of damage/failure of the whole system. This is expressed mathematically in Equation 2-2.

$$
R I=\frac{P_{f(d m g)}-P_{f(s y s)}}{P_{f(s y s)}}
$$

Where: $P_{f(d m g)}=$ probability of damage of a member

$$
P_{f(s y s)}=\text { probability of system failure/collapse }
$$

Several researchers, such as Ghosn and Moses (1997), incorporate the use of this system reliability based approach to calibrate and determine the appropriate deterministic redundancy limits. It should be noted that many of these approaches are integrally related to the respective methods used to determine system redundancy. For more description regarding these methods, see Section 2.5. 


\subsection{DEFINITIONS OF RELEVANT Limit STATES AND FAILURE CRITERIA}

In general, while the definitions of redundancy discussed in Section 2.2 vary among the bridge community, what is agreed upon is that the bridge's ability to resist loads in a damaged state is an important factor in determining its inherent levels of redundancy. Therefore, a review of the limit states that researchers have associated with redundancy and system capacity in a damaged state is in order to develop a means to adequately quantify redundancy. Discussed in this section are the limit states used in studies on bridge redundancy that were determined to be most critical.

\subsubsection{Individual Member Capacity}

One of the initial checks for redundancy used by many researchers is simply to perform a linearly elastic analysis to determine whether or not all members in a given bridge meet current AASHTO LRFD requirements for individual member capacity (i.e. whether they will fail or not under design loads). While this is not a direct redundancy evaluation, many of the proposed limit states are mathematically linked to this member-oriented evaluation (essentially expressing redundancy as the ratio of system reserve capacity to individual elastic member resistance under an applicable limit state). It should also be noted that, if a structure does not meet individual capacity requirements under elastic conditions, it is unlikely that it would be able to exhibit any appreciable level of redundancy.

In terms of system-level redundancy, once a structure has been found/designed to meet all applicable AASHTO limit states under minimum design loads, an additional linear analysis is then performed to determine how much additional load would cause the first member under tensile loads in the system to fail (i.e. the fracture-critical member). Ghosn and Moses (1997) relate this to the amount of live load on the system by incrementally increasing the magnitude of two HS20-44 trucks placed on the bridge. Once the first member reached its failure load, the magnitude of the load due to the augmented trucks would be divided by the initial magnitude to obtain a ratio (relating to individual member capacity) for later use in measuring redundancy. 


\subsubsection{Ultimate System Capacity}

As opposed to the previous limit state, the ultimate system capacity limit state evaluates the strength of the overall structural system rather than an individual element. In other words, individual member fractures or collapses are only of importance in this limit state when they are the contributing factor in overall system collapse or failure. However, the two limit states are similar in the analysis procedures with two exceptions: the first being that the structure be analyzed nonlinearly and the second is that the load is incrementally increased up until the point of system collapse. According to Galambos et. al (1992), the structure should respond to extreme magnitude events in such a way that the chance of a major failure of the structural system is acceptably small. NCHRP Report 406 further specifies this limit state as the maximum possible truck load that can be applied to the structure prior to collapse. Typically, this limit state is reached as the structure is unable to continue to carry additional loading which leads to significant amounts of displacement in the structure.

\subsubsection{Excessive Deflection (Functionality)}

One criterion commonly found in literature on bridge redundancy was a maximum permissible deflection limit for bridge superstructures. The limits are related to the safety (or functionality) of a bridge under large permanent deformations. While these large deformations may not necessarily lead to system or partial system collapse, they may render a bridge unfit for regular traffic. In addition, NCHRP Report 406 (Ghosn and Moses, 1997) suggests that possible functionality criteria may also include maximum rotation requirements; however, as rotations would only apply to flexural elements, displacement criteria are preferred.

It should be noted that, while a consensus exists among the bridge community that maximum permissible deflections (as they relate to bridge functionality) are an essential criterion by which to quantify bridge redundancy, no specific limit is uniformly specified. Many of the suggested deflection limits in research are proposed based solely on what a bridge user would find "visible" or "tolerable." Galambos et al. (1992) suggest a maximum permissible deflection (related to the shakedown limit of steel I-girders) of span/300, which is chosen as "the limit of visual observation." Ghosn and Moses (1997) used live load displacement as a functionality 
criterion and proposed a limit of span/100, which was believed to be the "maximum visible displacement that a bridge user or observer can tolerate." In research by Ghosn et al. (1994) (1992), a similar method is employed evaluating redundancy in terms of maximum permissible deflections, however limits in these projects are set at span/200 and span/300, respectively. In addition, Sandberg and Parmelee (1986), while recognizing the importance of a maximum deflection limit, chose not to specifically define a maximum value; instead, they only state that deflections should not impede the ability of a driver to control his/her vehicle.

Another deflection-related limit state that was found was instead related to maximum permissible slopes on bridge decks under member failure conditions. NCHRP 319 (1988) state that selection of an appropriate deflection limit should be based on the maximum allowable transverse and longitudinal rotations of bridge decks (as they relate to driver safety), where transverse slopes result from midspan fractures and longitudinal slopes result from worst-case fracture locations. In addition, Helba and Kennedy (1994) state that ultimate allowable loads

should be related to the ratio of permissible transverse deflections along the cross-section at the location of failure and the angle of the failure path along the transverse section.

\subsubsection{System Capacity under Damaged Conditions}

Relating very closely with the ultimate capacity limit state and the existence of fracturecritical components in a bridge (see Section 2.2.2.1), the system capacity limit state under damaged conditions varies from previous limit states in that it consists of the removal of a main load carrying component of the structure for analysis. Again, much like the ultimate limit state discussed in Section 2.3.2, a nonlinear analysis of the structure with HS-20 incremental truck loadings is performed to determine the system behavior while undergoing the complete loss of a component and to determine the resulting system capacity until collapse (NSBA, 2010).

\subsection{Findings of Previous Redundancy Studies}

As previously discussed, there is no consistently accepted definition for redundancy or the pertinent limit states associated with its determination. However, many research efforts 
throughout the years have been focused on evaluating degrees of redundancy in various structural systems. A review of these studies and their findings is essential in order to better understand structural redundancy and more adequately formulate a methodology to quantify it in highway bridges. Presented in this section is an overview of the previous findings in the area of bridge superstructure redundancy.

\subsubsection{Bridge Superstructures with Two Parallel I-Girders}

AASHTO LRFD Specifications (2010) state that any girder-type bridge with less than three girders is a non-redundant fracture-critical structure. However, the Task Committee on Redundancy of Flexural Systems (1985) states that a continuous structure with well-balanced spans will more than likely not collapse in the event of failure of one of the girders, even if the bridge is a two-girder system. Therefore, it is important to determine the levels of structural redundancy in a two-girder bridge, along with the checking of any alternate load paths that may be present. Often, the cause for failure of a main load carrying member in two-girder systems is related to fatigue cracking as a result of unstable crack growth (Ghosn and Moses, 1997). Hence, many of the research studies conducted on the redundancy of two-girder bridge systems are performed using numerical methods to simulate cracked girder scenarios, as this effect is difficult to test on in-place bridges.

Heins and Kato (1982) present the results of a deterministic, analytical study on the load redistribution of cracked girders in plate girder systems. To determine the effects of cracking with or without bottom lateral bracing, three scenarios were evaluated: uncracked girder, cracked girder without bottom lateral bracing, and cracked girder with bottom lateral bracing. Each scenario was applied to a bridge model consisting of four steel girders with a span of 120 feet. Acting conservatively, the web was assumed to have zero stiffness at the crack location which produced larger displacements and stresses than would actually occur in a structure. Two HS-20 trucks were used to simulate the loading effects, and dead load effects were neglected. As shown in Table 2.1, the bridge models with the bottom lateral bracing included, performed better than the models without the bottom lateral bracing as expected; however, the deflection and stress values without the bottom lateral bracing are well within an acceptable range when checked at an uncracked section of a cracked girder. 
Location

of Interest

\section{System Condition}

Uncracked Cracked (w/o Bracing) Cracked (w/Bracing)

Deflection at

Crack Location (in)

6.510

4.04

Maximum

Deflection

at Locations Away

0.765

0.980

1.214

from Crack (in)

Maximum Stress at

Locations Away

6.15

7.57

9.98

from Crack (ksi)

\section{Table 2.1: Two-Girder Redundancy Results (Heins \& Kato, 1982)}

The above results led the researchers to come to two significant conclusions. The first of these is that the system possesses significant load redistribution capabilities; for example, even when there is a crack in one of the girders and no bracing is present, the uncracked girder bending stress only increases by $1.42 \mathrm{ksi}$ and deflects 0.215 inches more than in its undamaged state. The second conclusion is that bottom lateral bracing members contribute significantly to the load redistribution of the system, specifically when evaluating the deflection values at the cracked section of the girder (decreasing the computed deflection by 2.47 inches). In general, the bracing members are able to "effectively create redundancy" in a two-girder system, even when a crack is present in one of the main members which can, in turn, prevent collapse of the structure. These results were further validated in a study by Idriss et al. (1993), where analyses were performed on a two-girder welded steel bridge with bottom lateral bracing. Following the same basic procedures as Heins and Kato, this study reinforces the positive effects of the bottom lateral bracing on the structure's load redistribution capabilities in a damaged condition. In addition, Idriss et al. found that an increase in the tensile forces present in the bracing members near the crack location were proven to provide additional stability for the structure.

A slightly modified approach, presented in NCHRP 319 (Daniels et al.,1988), determines the redundancy and rating of both simple and continuous spans as well as bridges exhibiting noncomposite or composite action. Finite element modeling was employed for deterministic and 
probabilistic analyses with fracture occurring at mid-span in one girder of the structure. Specifically, the fracture was introduced in the girder from the bottom flange through the full depth of the web. Much like the method performed by Heins and Kato, an incremental loading scheme is applied consisting of two AASHTO HS-20 truck loads. However, the modification to this approach is that full service dead load is applied to the structure, along with a 30 percent increase of the live load due to impact. Trucks were placed longitudinally and transversely to maximize force effects in the fractured girder. The two trucks were then incrementally increased until its maximum capacity is reached or deflections deemed intolerable are obtained. Two rating methods were employed to assess the redundancy: allowable stress and load factor.

Several key findings were observed in this research; the most essential conclusion being that a two-girder bridge structure possesses significant load redistribution capabilities, even after near full depth fracture, as long as the structure includes a redundant bracing system, such as bottom lateral bracing. For the simple span, an HS-60 was found to be its maximum capacity while an HS-50 represented maximum capacity for the continuous span. Therefore, simple spans were proven to exhibit higher after-fracture redundancy levels than continuous spans; this was attributed to the average cross section of the continuous span being smaller than that of the simple span.

\subsubsection{Bridge Superstructures with Multiple Parallel I-Girders}

Generally, a bridge structure with multiple girders is considered by many in the bridge community to be redundant. However, the specific level of load redistribution capabilities must be determined through analytical and experimental testing of such structures. Studies have been conducted with bridges having from two to ten girders. One such study by Sandberg and Parmelee (1986) performed on a three-girder system employed the same analysis procedure used by Heins and Kato (1982) for their two-girder analysis, with the exception of using two HS-25 trucks for the live load. The three-girder system results further demonstrated the importance of cross frames as the system was shown to have additional load redistribution capabilities due to the presence of cross frames.

A large number of redundancy studies are summarized in NCHRP Report 406 (Ghosn and Moses, 1997) or follow the same procedures and are included in alternative papers by Ghosn 
or Moses (1988) (1994) (1992). This research examined both steel and concrete bridge superstructures in several span length and girder configurations. For steel or concrete superstructures, the following parameters were considered: span lengths ranging from $45-150$ $\mathrm{ft}$, number of girders ranging from 4 to 10 , girder spacing between 4 and $12 \mathrm{ft}$, and concrete bridge deck thicknesses between 7.5 and 8.5 in. All of the studies were performed using the program NONBAN (Nonlinear Bridge Analysis Program), and both simple and continuous span bridges were investigated.

Out of the NCHRP Report 406 studies, 120 of the bridges analyzed were steel and comprised of grade A36 steel with a concrete deck having a compressive strength of $3.5 \mathrm{ksi}$. Similar to previously mentioned studies, an incremental loading procedure was utilized with two AASHTO HS-20 trucks placed such that the outside wheel of one truck was applied directly over the exterior girder. Additionally, the dead load of the structure was applied; however, since the primary goal was to determine each bridge's capacity, safety and impact factors were not applied. Analyses were performed for both the intact and damaged structure conditions in which the damaged condition involved removing one exterior girder from the structure with the assumption that it was unable to carry any part of the load.

For each condition (intact or damaged) and configuration analyzed, three deterministic system reserve ratios $R_{u}, R_{f}$, and $R_{d}$ are calculated and relate to the ultimate limit state, functionality limit state, and the damaged condition, respectively (for more description of these ratios, see Section 2.5.1). A system redundancy factor, $\varphi_{\mathrm{s}}$, is also calculated for each limit state.

$$
\begin{aligned}
& \phi_{S} \phi R=\gamma_{D} D+\gamma_{L} L \\
& \text { Where: } \phi_{S}=\text { system redundancy factor } \\
& \phi=\text { member resistance factor } \\
& R=\text { resistance capacity of the member } \\
& \gamma_{D}=\text { dead load factor } \\
& D=\text { dead load effect } \\
& \gamma_{L}=\text { live load factor } \\
& L=\text { live load effect }
\end{aligned}
$$$$
\text { Equation 2-3 }
$$

Out of the three chosen limit states, the lowest system redundancy factor controls for each configuration, with the ideal value being greater than or equal to 1.0. Figure 2.1 shows that, 
while the ultimate and functionality limit states produce fairly constant ratios, the damage limit state ratios decrease as the girder spacing increases. Thus, the damage limit state was found to be the most critical of those evaluated. From these system factor values, the most favorable layout for a two-lane, simple span steel I-beam bridge was found to be six girders spaced between 6 and $8 \mathrm{ft}$.

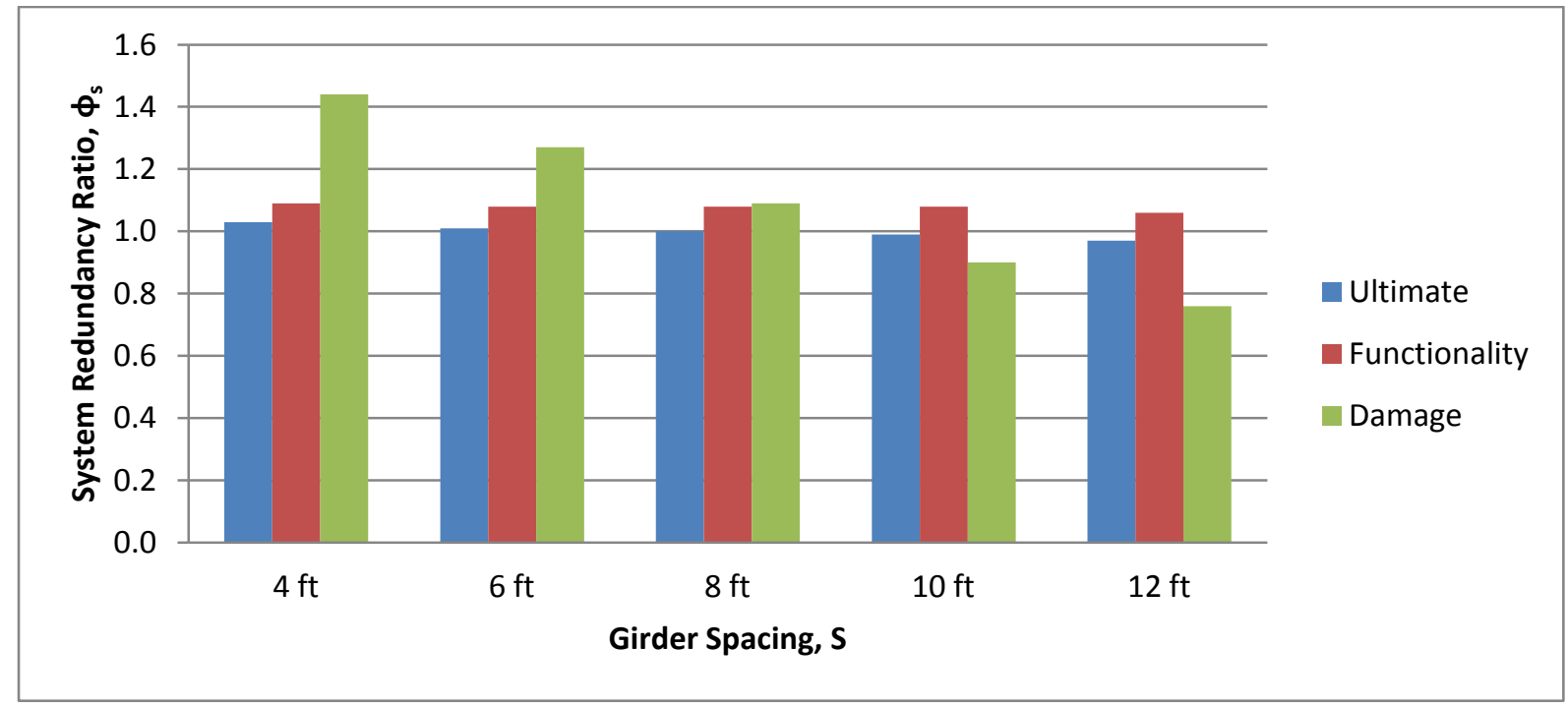

Figure 2.1: NCHRP Report 406 Results for Simple Span, Six Steel I-Girder Layouts

In addition, the continuous-span studies in NCHRP Report 406 indicated that steel girders with noncompact flanges and/or webs were fracture-critical and would be subject to sudden collapse or failure. On the other hand, continuous-span steel girder bridges with compact flanges and/or webs exhibited higher system redundancy ratios than simple-span bridges, however the difference between the two is minimal. Continuous span, steel I-girder redundancy ratio results are shown in Figure 2.2. 


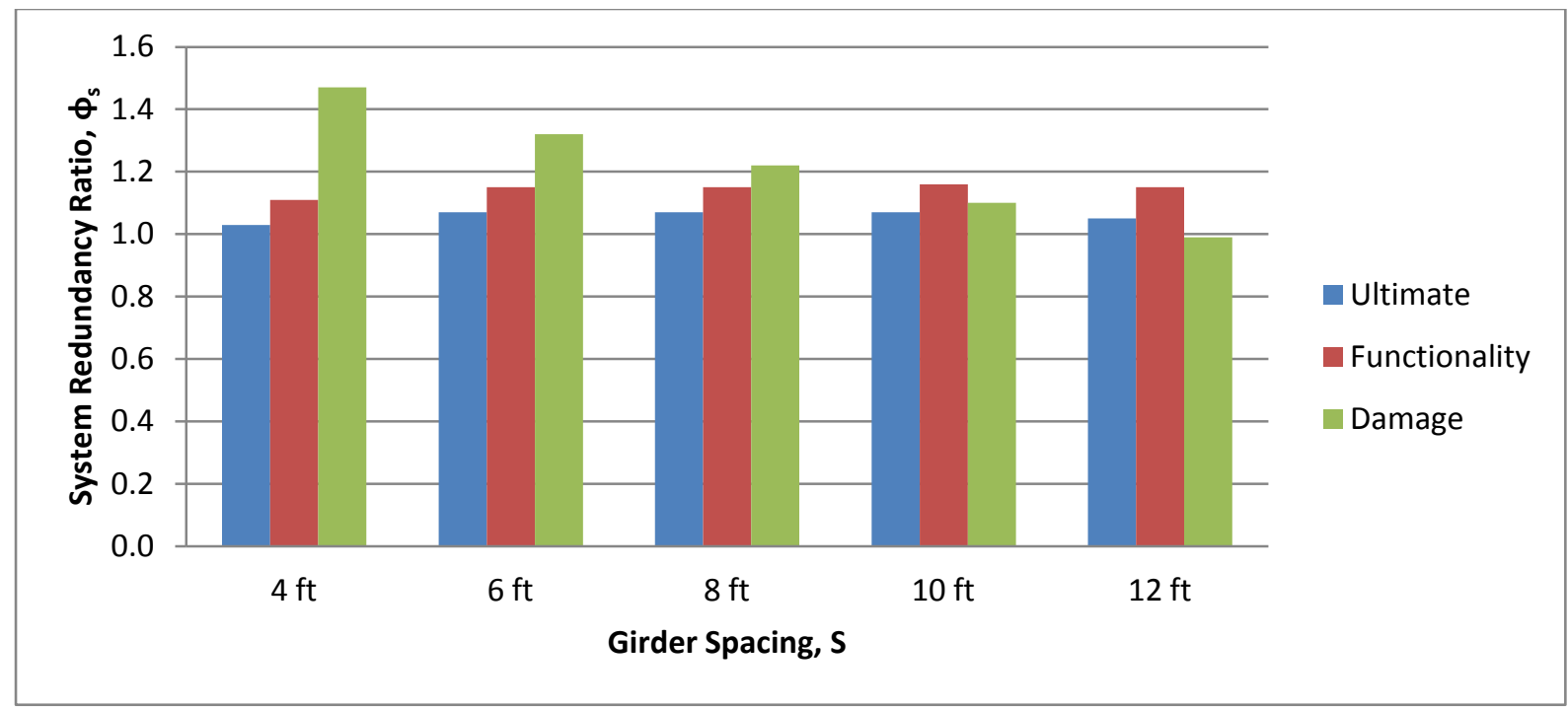

Figure 2.2: NCHRP Report 406 Results for Continuous Span, Six Steel I-Girder Layouts

There were also 102 simple-span and 51 continuous span prestressed concrete bridges evaluated in NCHRP Report 406 (Ghosn and Moses, 1997). For the beams in these bridges, concrete with a compressive strength of $5 \mathrm{ksi}$ was modeled; for the decks, $3.5 \mathrm{ksi}$ was employed. The previously described loading and analysis procedures were again employed to determine the level of system redundancy present in each configuration. Six girders spaced between 6 and $8 \mathrm{ft}$ was shown to be the most optimal configuration for simple-span concrete bridges, which was also found as the optimal configuration for the simple-span steel bridges. Continuous concrete bridge configurations were analyzed in a similar fashion as the continuous steel bridges with the addition of an assumption of constant reinforcement of the bridge deck for each configuration. The final outcome for continuous concrete bridges displayed that bridges with close beam spacing exhibited higher redundancy when continuity was present at the middle support.

Frangopol and Nakib (1991) present a study based upon alternate forms of damage models on a simple-span, steel girder bridge for both deterministic and probabilistic approaches. The bridge structure analyzed consisted of a 90 -foot single span comprised of four steel girders spaced at $9 \mathrm{ft}$ with a concrete deck thickness of 8.5 in. Dead load, two HS-20 truck loads, and impact loads were applied to the structure with an incremental application of live plus impact loadings as was employed in previous studies. This research was conducted using the commercial finite element software package Abaqus to evaluate two damage models: corrosion 
and accidental. To simulate corrosion, a specified amount of material is removed from the girder surface; while for the accidental model, up to two girders are completely removed from the bridge. For both damage models, the redundancy factor decreased as the amount of damage induced on the structure was increased; thus, the overall capability of load redistribution of the structure decreased as the damage increased.

\subsubsection{Bridge Superstructures with Parallel Box Girders}

Bridge configurations for box girder structures have also been evaluated by previous researchers. In addition to the multiple I-girder studies included in NCHRP Report 406 (Ghosn and Moses, 1997), prestressed concrete spread box girder and multi-box beam bridges were designed and evaluated for redundancy. Spread box girder bridges were designed with varying parameters for 45 total bridge configurations with varying spans of $60 \mathrm{ft}, 100 \mathrm{ft}$, and $150 \mathrm{ft}$. For each span length, the number of girders, girder spacing, and deck thicknesses were parametrically varied as shown in Table 2.2 .

\begin{tabular}{c|c|c|c|} 
Spacing & $8 \mathrm{ft}$ & $15 \mathrm{ft}$ & $25 \mathrm{ft}$ \\
Number of Beams & $2,3,5$ & $2,3,5$ & $2,3,5$ \\
Deck Thickness & $8 \mathrm{in}$ & $9 \mathrm{in}$ & $10 \mathrm{in}$
\end{tabular}

Table 2.2: Bridge Design Parameters

The program NONBAN is utilized to evaluate the bridges under the loading conditions described in Section 2.4.2 for the NCHRP Report 406 multiple girder studies. Following the same procedures used in the multiple girder studies, load factors were calculated for the ultimate, functionality and damaged limit states, which were then used to determine the system redundancy ratios. Overall, the results showed shorter span bridges, in which the beams are closely spaced, to be more redundant than bridges with longer spans or beams spaced further apart. An adequate redundancy factor is a value above 1.0 (see Section 2.4.2); these factors for the $60 \mathrm{ft}, 100 \mathrm{ft}$, and $150 \mathrm{ft}$ spans are given in Tables 2.3, 2.4, and 2.5, respectively. 


\begin{tabular}{c|ccc|ccc|ccc}
$\begin{array}{c}\text { Spacing } \\
\varphi_{\mathrm{s}}\end{array}$ & \multicolumn{3}{|c|}{$8 \mathrm{ft}$} & \multicolumn{3}{c|}{$15 \mathrm{ft}$} & \multicolumn{3}{c}{$25 \mathrm{ft}$} \\
\hline \hline 2 beams & Ultimate & Funct. & Damage & Ultimate & Funct. & Damage & Ultimate & Funct. & Damage \\
\hline beams & 1.00 & 0.99 & 1.38 & 0.96 & 0.94 & 1.32 & 0.95 & 0.91 & 1.24 \\
5 beams & 1.01 & 1.00 & 1.52 & 0.99 & 0.96 & 1.34 & 0.97 & 0.94 & 1.27 \\
& 1.04 & 1.02 & 1.60 & 1.01 & 0.94 & 1.47 & 0.97 & 0.93 & 1.41
\end{tabular}

Table 2.3: System Factors for $60 \mathrm{ft}$ Span

\begin{tabular}{c|ccc|ccc|ccc|}
$\begin{array}{c}\text { Spacing } \\
\varphi_{\mathrm{s}}\end{array}$ & \multicolumn{3}{|c|}{$8 \mathrm{ft}$} & \multicolumn{3}{|c|}{$15 \mathrm{ft}$} & \multicolumn{3}{c|}{$25 \mathrm{ft}$} \\
\hline \hline 2 beams & 0.98 & 0.95 & 1.30 & 0.93 & 0.91 & 1.24 & 0.93 & 0.88 & 1.16 \\
3 beams & 0.99 & 0.96 & 1.41 & 0.95 & 0.91 & 1.32 & 0.94 & 0.90 & 1.23 \\
5 beams & 1.04 & 0.99 & 1.45 & 1.02 & 0.93 & 1.37 & 0.97 & 0.93 & 1.33
\end{tabular}

Table 2.4: System Factors for 100 ft Span

\begin{tabular}{c|ccc|ccc|ccc|} 
Spacing & \multicolumn{3}{|c|}{$8 \mathrm{ft}$} & \multicolumn{3}{|c|}{$15 \mathrm{ft}$} & \multicolumn{3}{c|}{$25 \mathrm{ft}$} \\
$\boldsymbol{\varphi}_{\mathrm{s}}$ & Ultimate & Funct. & Damage & Ultimate & Funct. & Damage & Ultimate & Funct. & Damage \\
\hline \hline 2 beams & 0.96 & 0.94 & 1.21 & 0.95 & 0.92 & 1.17 & 0.95 & 0.90 & 1.12 \\
3 beams & 0.97 & 0.94 & 1.26 & 0.95 & 0.92 & 1.24 & 0.95 & 0.91 & 1.19 \\
5 beams & 0.99 & 0.96 & 1.41 & 0.98 & 0.92 & 1.33 & 0.96 & 0.92 & 1.26
\end{tabular}

Table 2.5: System Factors for 150 ft Span 
NCHRP Report 406 further investigates box girder bridges with an evaluation of three multi-box beam bridges. These bridges consisted of noncomposite, $70 \mathrm{ft}$. span bridge with a slab thickness of 5.5 in. Configurations with 8, 10 and 11 box girders were designed with box dimensions having sections with 4 foot width and 33 inch depth.. NONBAN, as well as the loading and analysis procedures explained in Section 2.4.2, was utilized for the multi-box girder studies also. As shown in Figure 2.3 the multi-box beam bridge studies exhibited load redistribution capabilities overall; although the configuration with 10 boxes proved to be the most optimal.

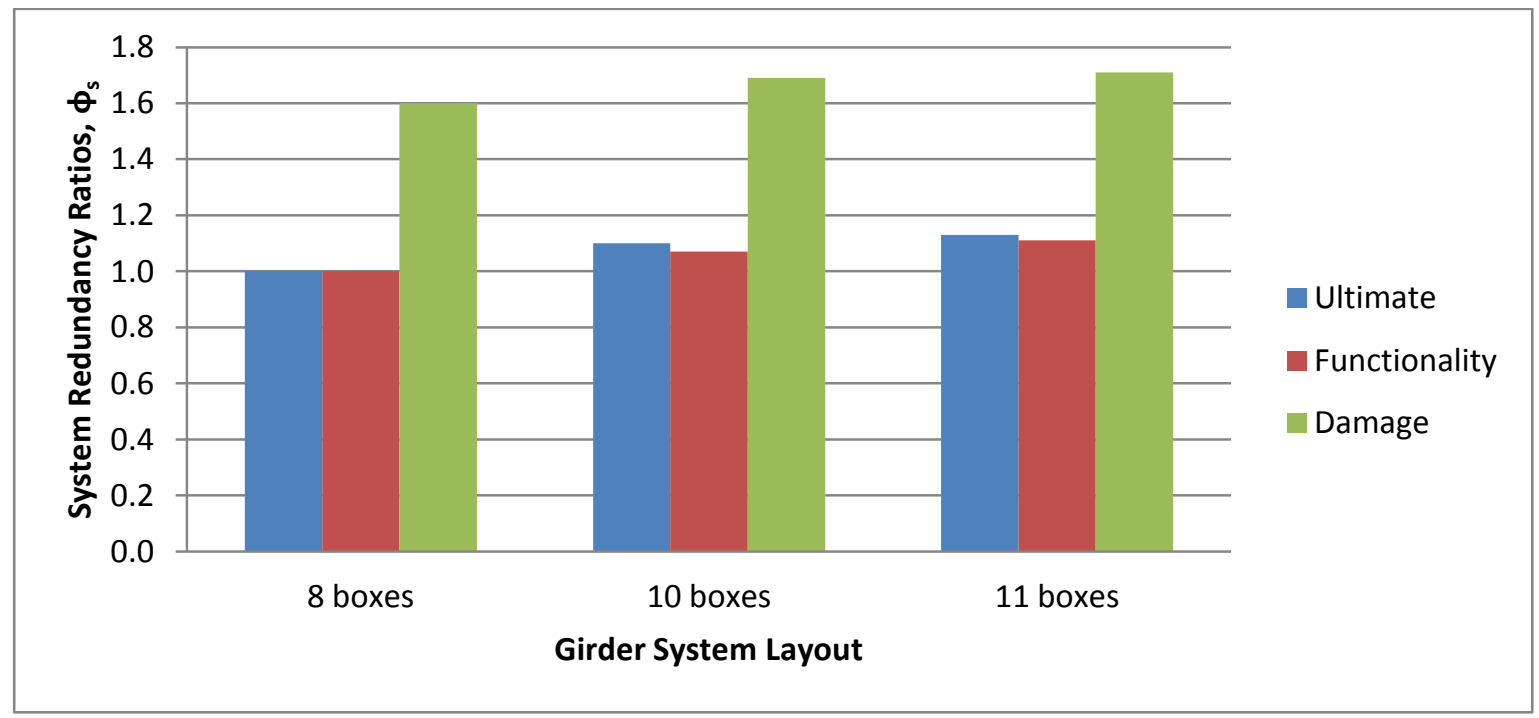

\section{Figure 2.3: NCHRP Report 406 Results for Simple Span, Steel Box Girder Layouts}

Another box girder study was performed by Hovell (2008) on steel twin trapezoidal box girders that were previously in service in a simple-span portion of an HOV lane in Texas. The $120 \mathrm{ft}$. long girders with an 8 in. deck thickness were reconstructed on concrete piers to enable completion of a load test. Constant thickness plates provide internal diaphragms at support points. In addition, the two girders are connected with diaphragms and bracing in several forms: vertical stiffeners, internal stiffener trusses, top lateral bracing and external construction bracing. To simulate an HS-20 truck loading condition, concrete girders with blocks in place for the axles were placed on the bridge located $2 \mathrm{ft}$ from the concrete parapet such that maximum bending moment was produced in one of the boxed (see Figure 2.4). Fracture was then induced in the bottom flange of this box through the use of explosives. 


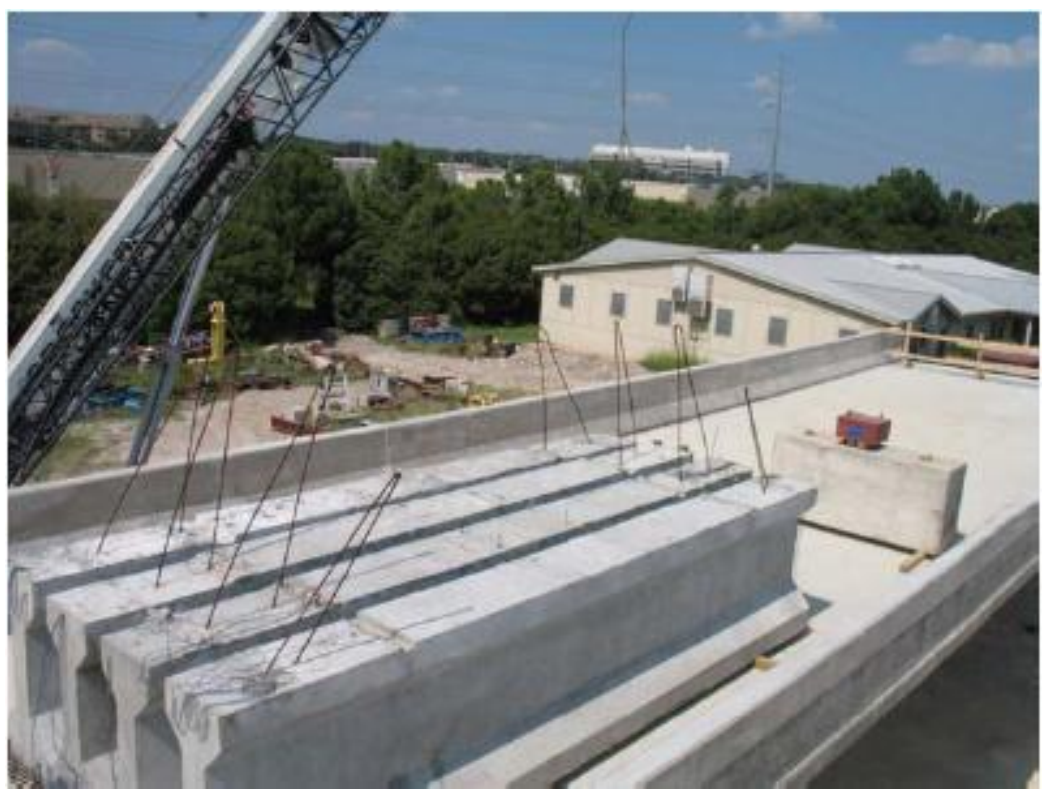

Figure 2.4: Concrete Girders Used to Simulated HS-20 Live Loading (Hovell, 2007)

Following the field testing, an Abaqus finite element model of a multi-span, continuous bridge was created with a dynamic loading applied to evaluate structural redundancy. It was shown that the multi-span, continuous structure did have higher redundancy than the simple span counterpart. However, in the model the cross section of the girders was kept the same for both simple and continuous spans; in most cases, a multi-span structure would incorporate smaller cross sections than that of a simple span bridge. While further examination of the continuous span's structural redundancy was to be continued, preliminary investigation suggested that the structure was determined to be redundant and to exceed the requirements set forth in AASHTO (2010).

\subsubsection{Steel Truss Bridge Superstructures}

Although redundancy in steel truss bridges is an increasingly prevalent issue, research on such structures is limited when compared with the research available in the previous sections pertaining to beam structures. Following the Point Pleasant, WV bridge collapse, the redundancy of 2-eyebar members has been further evaluated. Bondi (1985) assessed these members in a section of a two lane, 2,000 foot long bridge; overall, the bridge includes five 
through-truss spans, two deck girder spans, two multi-girder spans, and one pony truss span. This bridge is shown below in Figure 2.4.

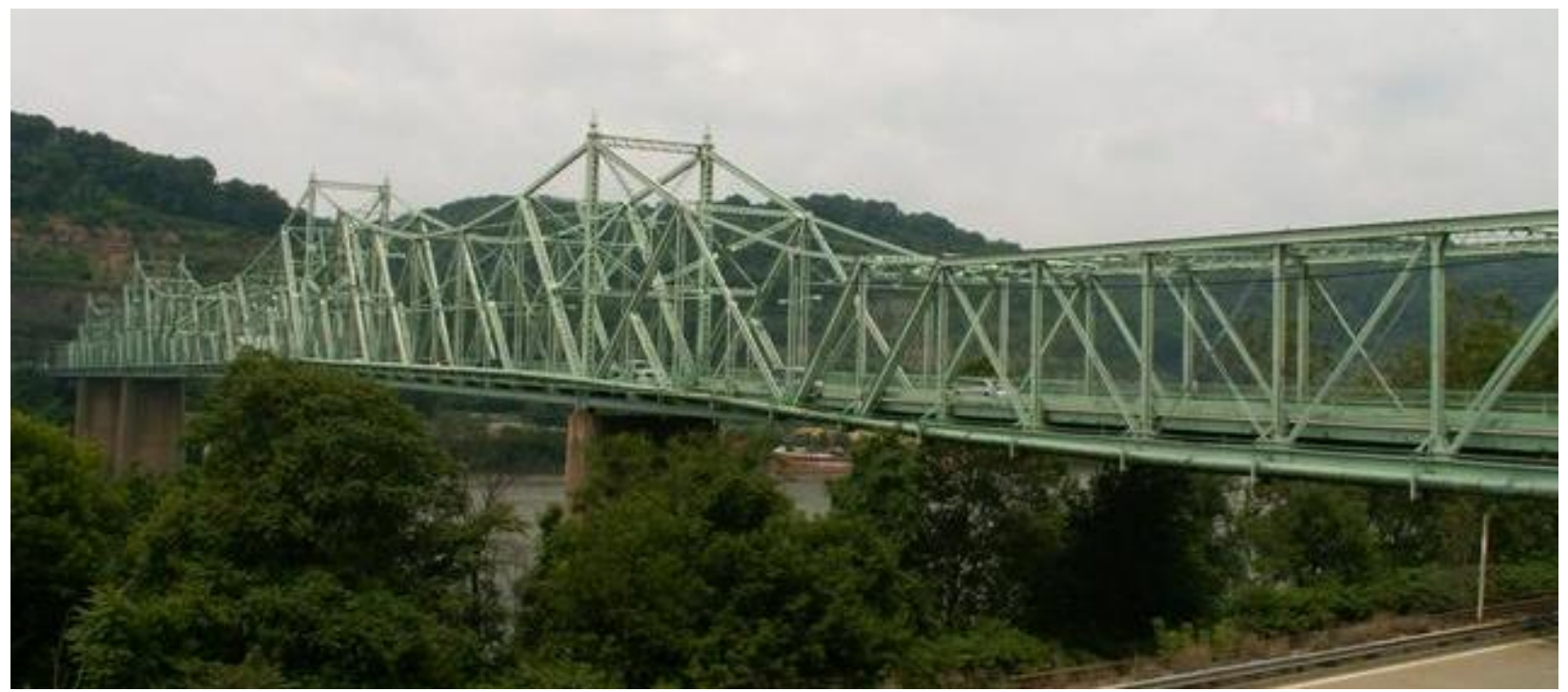

Figure 2.5: Ambridge-Aliquippa Bridge (Holth and McOmber, 2006)

Specifically focusing on a section of the cantilever truss portion of the through truss spans, this investigation was further centered around the 2-eyebar, heat treated members and their tendency to undergo stress corrosion and corrosion induced fatigue cracking which could result in failure of the entire structure. A thorough visual inspection of the structure was completed to determine the current conditions of bridge members. In addition, a redundancy analysis was performed for each 2-eyebar member in order to assess the damaged system's response to the failure of these members.

These investigations revealed that the failure of any one of 722 -eyebar members in the structure would cause collapse of the whole system. Based on these findings, a comprehensive rehabilitation was performed to strengthen the 2-eyebar members. Requirements for the new members included a safety factor of at least 2.5 which was believed to add to the load redistribution capability and the overall redundancy of the structure. Each 2-eyebar member in all 72 locations was replaced with I-beams and angles, which consisted of over a year of construction work and a total cost of 2.3 million dollars.

Another truss bridge type, a post-tensioned truss bridge, was analyzed in research by Ayyub and Ibrahim (1990). This study incorporates three layouts for the truss tendons, shown in 
Figure 2.6: straight tendon, one-drape tendon, and two-drape tendon. Analysis of these tendon layouts was performed in three stages: the dead load alone, the dead plus the post-tensioning loads, and the live and impact loads combined. In each stage, the force in the tendon was assumed to stay constant along the tendon and to be in tension only. Analysis results were determined by superimposing the second and third stage results and in the calculation of a redundancy factor, $\psi$, which represents the ratio of the average system probability of failure before post-tensioning to the average system probability of failure after post-tensioning. For this evaluation, a value of $\psi$ greater than 1.0 signifies a post-tensioning configuration capable of load redistribution.
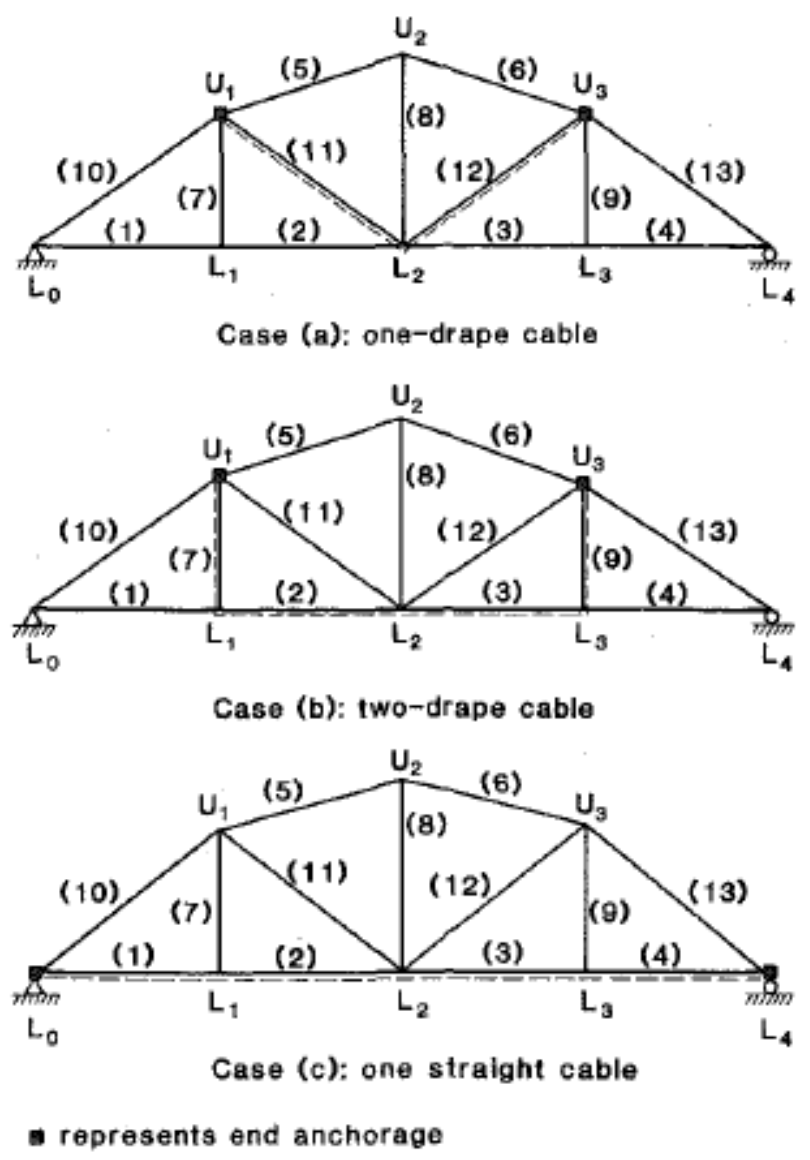

Figure 2.6: Post-tensioned tendon layouts (Ayyub and Ibrahim, 1990)

Each of the tendon layouts were found to produce a truss capable of load redistribution; however, each damaged condition resulted in a different alternate load path. In addition, posttensioning was proven to reduce the forces in all truss members in each of the three tendon layouts, which contributes to the load redistribution capability of the truss bridge system. Table 
2.6 displays the effects of posttensioning in terms of the calculated redundancy factors for this study.

\begin{tabular}{c|c} 
Truss System & Redundancy Factor, $\boldsymbol{\psi}$ \\
\hline \hline Original Truss & 1.0000 \\
Straight Tendon & 1.0023 \\
One-Drape Tendon & 1.6905 \\
Two-Drape Tendon & 3.6056
\end{tabular}

Table 2.6: Effects of Posttensioning on Redundancy Factors

A study performed by the Bridge Engineering Software and Technology (BEST) Center at the University of Maryland (2000) analyzed the degrees of redundancy present in a simplespan, steel through truss bridge. The bridge analyzed, U.S. Bridge Corporation Bridge 3000, is a 90 foot bridge comprised of 6 panels with an 8 inch concrete deck and a 20 foot roadway width. For numerical analysis, the bridge was idealized as a three-dimensional finite element model in ANSYS 5.6. The model used BEAM24 elements, which is a uniaxial element with axial, bending, and St. Venant torsional capabilities

In addition to performing damaged condition analyses in accordance with NCHRP Report 406 (See Section 2.5.1), the bridge was also assessed according to the provisions of the Pennsylvania Department of Transportation (PennDOT) Design Manual, Part 4 (DM4) (2007). PennDOT outlines design provisions in addition to those in the AASHTO LRFD Specifications (2010) specifically related to structural redundancy.

Along with various guidelines for designing redundant structures, the DM4 also includes extreme event load combinations specifically focused toward evaluating fracture-critical elements. The Extreme Event III is described as the "load combination relating to the failure of one element of a component without the failure of the component." Also, the Extreme Event IV is described as the "load combination relating to the failure of one component without the collapse of the structure." It should be noted that the commentary states that Extreme Events III and IV are uncalibrated load combinations. Additionally, for Extreme Event IV, it is specified that a three-dimensional analysis is required, where the objective of this analysis is survival of the bridge (i.e., the bridge may have large permanent deflections, but it has not collapsed). 
Essentially, the authors performed the same type of analyses described in NCHRP Report 406 (see Section 2.5.1). The only difference in this study is that for the analyses, the Extreme Events III and IV described in the PennDOT DM4 were employed. For each case analyzed, the full dead load along with live load plus impact were applied, where the live load consisted of two lanes of an AASHTO HS-20 truck loading (which is the same as the PennDOT PHL-93 loading). The truck loadings were placed transversely to maximize loading on one truss line and longitudinally at the centerline and the one-third span locations with the maximum stress values recorded between the two locations. Impact and distribution factors were applied as specified by AASHTO.

To determine the level of redundancy present in the structure, as per NCHRP Report 406, a series of member type modifications as well as cut-chord analyses (to simulate damaged conditions) were performed in ANSYS. Specifically, the members' cross-sectional areas were modified to simulate internal member failure, and the members were removed (or cut) to simulate total member failure. Twelve fracture-critical members were identified; as a symmetric structure with only one line of truss being maximized, three bottom chord members and three diagonal members were chosen to comprise the cut-chord analysis cases. In total, eleven separate cases are analyzed, looking at various permutations of member modifications and cut chord analyses.

Overall, three of the eleven cases were classified as nonredundant load paths (according to the chosen limit states) where each of these cases involved a cut chord. However, while they were classified as nonredundant, the models did not experience collapse; instead, they failed the limits set forth for deflection and damaged condition capacity. In addition, each of the cases in which a member type was modified as well as the other three of the cut-chord cases were found to be classified as redundant. The fracture-critical members are shown in the figure below: 


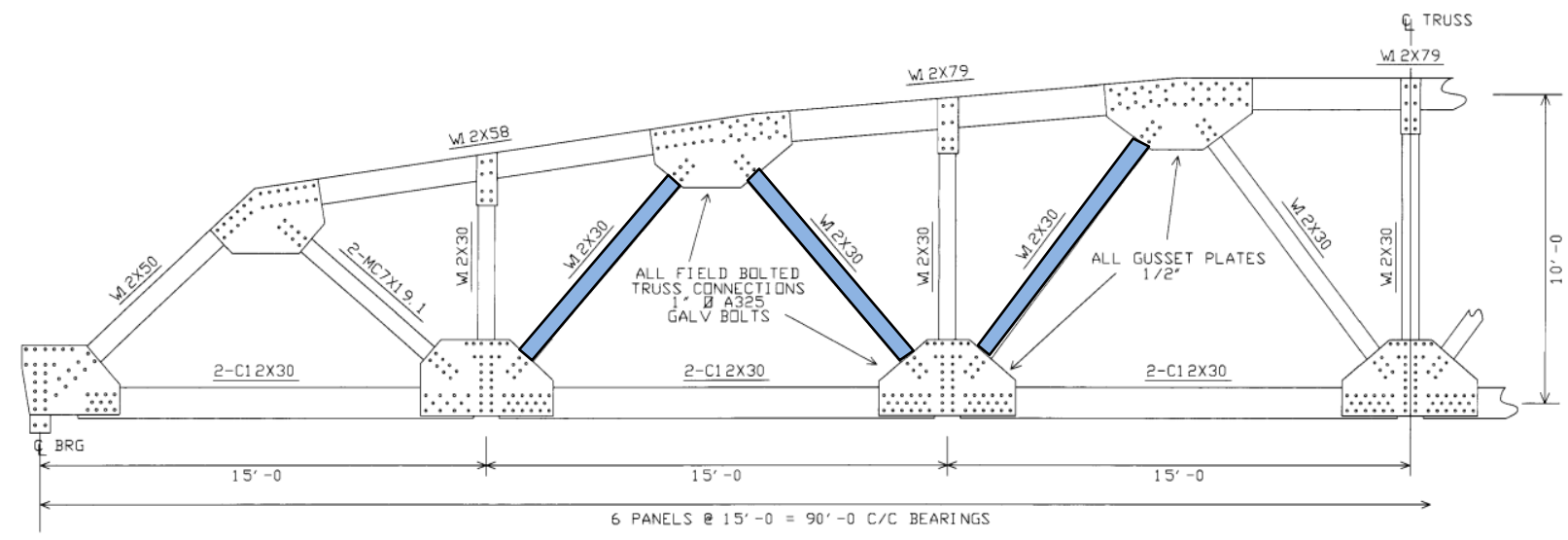

Figure 2.7: Fracture-critical members in U.S. Bridge Series 3000 (BEST, 2000)

\subsection{METHOdS FOR QUANTIFYING REDUNDANCY IN HighWAY BRIDGE SUPERSTRUCTURES}

In the previous section, various research projects were discussed, highlighting both the parameters that were investigated and their respective effects on structural redundancy. While many of the outcomes of these studies contained similarities, many of the methods used to quantify bridge redundancy (particularly analytical methods) contain slight variations from one another. This section summarizes various methods used for quantifying redundancy in bridge systems from previous work.

\subsubsection{NCHRP Report 406}

Ghosn and Moses (1997) presented a very rigorous method for determining redundancy in highway bridge superstructures. The main target of this methodology was to integrate system redundancy evaluation into the traditional member-oriented approach present in the Specifications. This was accomplished by deriving and calibrating "system factors" that could be used to modify individual member capacity in order to capture the system redundancy effect. Specifically, the formulation of their methodology revolved around the following goals:

1. Determining the limit states relevant to bridge redundancy.

2. Determining the level of loads that must be carried by the structure before reaching these limit states. 
3. Determining the types of damaged conditions that the structure can exhibit.

4. Including uncertainties in the analysis methodology.

For the methods prescribed in NCHRP Report 406, the authors employed four limit states. These limit states are as follows:

1. Member Failure: This is an individual member safety check using a linear elastic analysis. The individual member forces are then compared with the member capacities, as defined in the current specifications. This limit state was proposed in an effort to be consistent with current member-by-member evaluation techniques. Specifically, the truck loads are incremented until first member failure is reached. The "multiplier," or the scaled magnitude of the two trucks over the original magnitude, is referred to as $\mathrm{LF}_{1}$, and is incorporated later on in this method.

2. Ultimate Capacity: This is defined as the maximum capacity of the system and/or the load magnitude required to develop a collapse mechanism. Specifically, the same process of incrementing the truck loads is applied until the system collapses. However, since system collapse is directly related to the nonlinear material properties of the system (i.e. crushing of concrete, yielding of steel, etc.), a nonlinear analysis must be performed. The multiplier for this limit state, which is used later in this method, is referred to as $\mathrm{LF}_{\mathrm{u}}$.

3. Functionality Conditions: This is defined as the capacity of the system to resist a longitudinal live load displacement equal to the span/100. It is noted by the authors that, while excessive deflections may not lead the system to collapse, they may render the bridge unsafe for traffic (see Section 2.3.3). For this step, the bridge is again analyzed using a nonlinear analysis as deflections of this magnitude will most likely occur during a state of permanent deformations. The trucks loads are again incremented to the critical value, and multiplier for this limit state is referred to as $\mathrm{LF}_{\mathrm{f}}$.

4. Damaged Conditions: This is defined as the capacity of the system after the removal of a main load-carrying component. This member could be a previously identified fracture-critical member or a member that is susceptible to other types of damage, such as collisions by vehicles or boat traffic. The truck load is once 
again incremented in a nonlinear model until system collapse occurs, with the multiplier for this limit state being referred to as $\mathrm{LF}_{\mathrm{d}}$.

Next, since redundancy is generally defined in NCHRP Report 406 as the capacity after various levels of damage, system reserve ratios are calculated. Specifically, these are the ratios of the limit state capacities as they relate to the first limit state, that of member failure.

$$
\begin{gathered}
R_{u}=\frac{L F_{u}}{L F_{1}} \\
R_{f}=\frac{L F_{f}}{L F_{1}} \\
R_{d}=\frac{L F_{d}}{L F_{1}}
\end{gathered}
$$

Equation 2-5

Where: $R_{u}=$ system reserve ratio for the ultimate limit state

$$
\begin{aligned}
& R_{f}=\text { system reserve ratio for the functionality limit state } \\
& R_{d}=\text { system reserve ratio for the damaged condition }
\end{aligned}
$$

Essentially, these ratios quantify the amount of load redistribution capability of a given structure. For example, the first system reserve ratio, $\mathrm{R}_{\mathrm{u}}$, relates the system's capacity before collapse to the capacity of the individual members of the system. If these two quantities were equal (i.e. if $R_{u}=1.0$ ), it would indicate that the once first member failure occurred, the structure would also reach its collapse load or limit state associated with $F_{u}, F_{f}$, and $F_{d}$ respectively. Such a system would be classified by many as nonredundant.

It should be noted that caution must be exercised when employing this method. $\mathrm{R}_{\mathrm{f}}$, the system reserve ratio in Equation 2-4, relates the system's capacity before the maximum allowable deflection is reached to the capacity of the individual members of the system. If this value is found to be less than 1.0, it would indicate that the maximum deflection was reached before first member failure. However, this may result from the different procedures used to perform these analyses; a nonlinear analysis is employed in the functionality condition analysis whereas a linear analysis is used in the individual member evaluation. 
While this method provides engineers with a straightforward method for determining system-level redundancy ratios in a bridge, limits for these values are necessary in order to make design checks. Therefore, in addition to this deterministic method of analysis, NCHRP Report 406 also prescribes a reliability-based approach to determining system-level reliability. This approach is used to calibrate the limits for the system reserve ratios expressed in Equation 2-3 through Equation 2-5. It should be noted that, while most practicing engineers would not perform this type of probabilistic-based analysis, it is critical to the development of acceptable limits for these values in order to ensure an adequate and uniform level of safety in the method. Furthermore, it should be added that similar methods were used to calibrate the load and resistance factors present in the AASHTO LRFD Specifications.

Much like the previously defined deterministic approach, this reliability-based method is a function of the magnitude of applied live loads. These loads are broken down into two load cases: extreme loading cases and ordinary loading cases. The extreme loading case correlates to the maximum live load that is statistically likely to occur over the design life of the bridge. The AASHTO LRFD Specifications were developed for a design life of 75 years (2010); therefore, this is the time period that corresponds to extreme load scenarios. In this reliability analysis, like the loads used in the deterministic approach, the values are expressed as multiples of two HS 2044 trucks placed side-by-side on the deck. The values that are selected for this load are identical to the ones incorporated in the original calibration of the AASHTO Specifications; specifically these are expressed as the statistical mean of the live load "multiplier", $\overline{\mathrm{LL}_{75}}$, and the coefficient of variation (COV) of the multiplier, $\mathrm{V}_{\mathrm{LL}_{75}}$. Ordinary loading cases, however, are instead expressed as the statistical average of live loads that would occur over a two-year period. This was chosen since this represents the two-year inspection period that is mandatory for U.S. highway bridges according to the NBIS (2005). This statistical variable is similarly expressed as the statistical mean of the live load "multiplier", $\overline{\mathrm{LL}_{2}}$, and the $\mathrm{COV}$ of the multiplier, $\mathrm{V}_{\mathrm{LL}_{2}}$.

In reliability analyses, like the method employed in NCHRP Report 406, and the ones used to calibrate the AASHTO LRFD Specifications, the measure of safety used is referred to as the reliability index, $\beta$. A general definition of a reliability index can be expressed by $p_{f}=\Phi(-\beta)$, where $\mathrm{p}_{\mathrm{f}}$ is the probability of failure, and $\Phi(\mathrm{x})$ is the CDF of the normal (Gaussian) distribution. The AASHTO Specifications for main members were calibrated to produce a reliability index equal to 3.5 , which would correlate to a $0.023 \%$ probability of failure. Much like the previously 
described deterministic approach in calculating live load multipliers, this reliability-based method involves computing reliability indices for each of the applicable limit states. Expressions for these reliability indices are as follows:

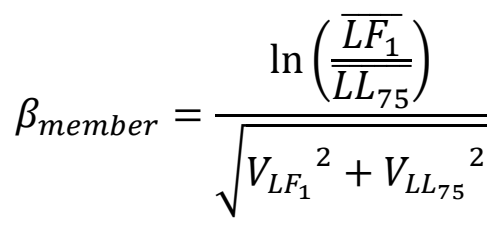

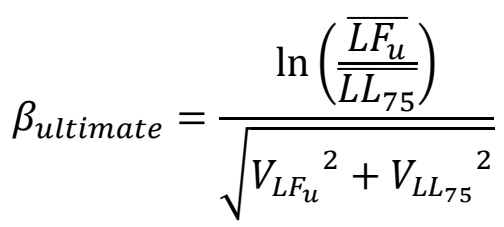

$$
\begin{aligned}
& \beta_{\text {functionality }}=\frac{\ln \left(\frac{\overline{L F_{f}}}{\overline{L L_{75}}}\right)}{\sqrt{{V_{L F_{f}}{ }^{2}+V_{L L_{75}}^{2}}^{2}}}
\end{aligned}
$$

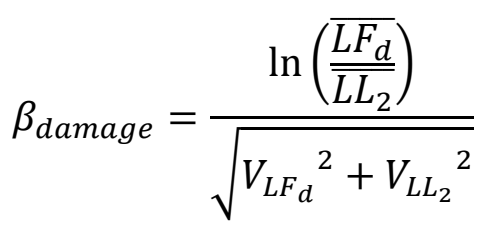

Where: $\beta_{i}=$ reliability index for the " $\mathrm{i}$ "th" limit state

$$
\begin{aligned}
& \overline{L F_{l}}=\text { statistical mean of "ith" live load multiplier } \\
& V_{L F_{i}}=\mathrm{COV} \text { of " } \mathrm{i} \text { " th" live load }
\end{aligned}
$$

According to the NBIS (as previously stated), the standard inspection period for bridges in the U.S. is two years. Therefore, since two years is the maximum likely period that damage to a structure would go unnoticed, the statistical variables related to live load in Equation 2-9 correspond to average live load conditions. All other limit state expressions, such as functionality and ultimate capacity, employ statistical variables related to extreme live load conditions.

Next in this probabilistic approach, similar to the calculation of system reserve ratios in the previously described method, relative reliability indices are calculated. Like system reserve 
ratios, these relative reliability indices can be thought of as probabilistic levels of redundancy, or measures of safety against first member failure.

$$
\begin{aligned}
\Delta \beta_{u} & =\beta_{\text {ultimate }}-\beta_{\text {member }} \\
\Delta \beta_{f} & =\beta_{\text {functionality }}-\beta_{\text {member }} \\
\Delta \beta_{d} & =\beta_{\text {damage }}-\beta_{\text {member }}
\end{aligned}
$$

To perform the reliability calibration on these limits, a matrix of slab-on-beam bridges (steel girders, prestressed concrete box beams, prestressed concrete I-beams, etc.) were analyzed to assess various parameters' impact on reserve redundancy indices. It should be noted that, for these analyses, since two-girder and even three-girder systems are usually considered nonredundant by most engineers, the analyses discussed were limited to systems with a minimum of four girders. However, as discussed in Section 2.4, even two-girder systems can possess levels of redundancy beyond expectations.

From these calibration procedures, the following values were selected as target relative reliability indices (in other words, redundant bridges would be expected to have $\Delta \beta_{i}$ values greater than these):

- $\Delta \beta_{u}=0.85$

- $\Delta \beta_{f}=0.25$

- $\Delta \beta_{d}=-2.70$

Using these target reliability index values, Equations 2-6 through 2-9 are employed, and $\overline{L F}_{l}$ are computed. These average values are then used to determine target system reserve ratios for use in the previously discussed deterministic approach. The following target system reserve ratios were proposed as follows:

- $R_{u} \geq 1.30$

- $R_{f} \geq 1.10$

- $R_{d} \geq 0.50$

It can be inferred that from these values what target system performance designers should expect from redundant bridge systems (or rather, systems that meet the target system reserve ratios). For example, from the first limit, designers should expect that the bridge could sustain a 
live load 30\% higher than the load that would cause the first member to fail. Also, from the last limit, an engineer would expect a bridge to be able to withstand $50 \%$ of its live load design capacity after the damage/failure of its most critical element.

\subsubsection{NCHRP Report 319}

Daniels et al. (1988) performed analyses focusing on levels of system redundancy as they relate to the bracing systems in two-girder steel highway bridge structures (see Section 2.4.1). Specifically, the authors attempted to determine the criteria associated with bracing systems and their effect on after-fracture redundancy and to establish general guidelines for designing for and retrofitting a bridge structure for after-fracture redundancy. To accomplish this, previous case studies on two-girder systems with and without bracing were gathered to determine a uniform methodology for assessing after-fracture redundancy. Four design and rating possibilities were considered based on the following strategies:

1. The design of an alternate load path to provide redundancy in a new bridge.

2. The design of a new alternate load path to provide redundancy in an existing or rehabilitated bridge.

3. The retrofit design of an as-built or existing alternate load path to provide redundancy in an existing or rehabilitated bridge

4. The analysis of an as-built or existing alternate load path to determine the afterfracture redundancy rating of an existing bridge.

While these guidelines alone do not depend upon the specific locations of fracture in a girder, the location of an alternate load path may be altered depending upon such fractures. Therefore, it is necessary to concentrate on any present fractures which tend to occur in the following locations:

- groove-welds and ends of welded cover plates on tension flanges

- ends of various reinforcement or attachment plates welded on girder flange or web

- diaphragm connections and end connections of floor beams or diaphragms

- floor beam brackets 
- top and bottom lateral bracing connections to girders

- transverse stiffeners, tack welds, and plug welds.

The ultimate goal of this effort was to assess or determine a redundant bracing system that would provide an alternate load path configuration that would act within the elastic range to provide after-fracture redundancy. These redundant bracing systems, in accordance with AASHTO requirements, would incorporate either bottom/top lateral bracing or diaphragms. In addition, such redundant bracing systems must specifically be configured to meet more rigorous requirements outlined by the authors. The top and bottom lateral bracing would require a horizontal truss configuration with the ability to resist any applied loads through the diagonal members in the truss bracing system. For the diaphragms, both full depth interior and end types would be present which would consist of either cross bracing, frames or trusses.

To evaluate such a bracing system as part of a bridge structure and design for system redundancy, the following three strategies were presented: design of an alternate load path which provides redundancy in a new bridge, design of a new alternate load path which provides redundancy in an existing bridge, and retrofit design of an as-built or existing alternate load path which provides redundancy in an existing bridge. For each of these strategies, a model is developed to represent either the bridge currently being designed or a bridge scheduled for retrofitting. This model is then subjected to dead, live, and impact loading arrangements using the rating vehicle as given in AASHTO. In addition, for vehicular live loads, the authors suggest that the number of lanes be reduced to appropriately represent the lower probability that the bridge experience full design live loads under damaged conditions. Furthermore, as damaged bridges are expected to experience deflections larger than those predicted in design (using a member-oriented approach), the authors propose an impact factor $\mathrm{I}=1.30$ for damaged scenarios.

The first step in evaluating a given model for redundancy is to check the primary members and connections in the structure during damaged conditions with the load and resistance factor method (with the inclusion of the proposed impact factor). Next the serviceability or deflection criteria are checked much like the functionality conditions specified in Section 2.5.1. Maximum deflection-to-span length $(\Delta / \mathrm{L})$, maximum longitudinal girder slope $\left(\theta_{\text {long. }}\right)$, and maximum deck transverse slope $\left(\theta_{\text {tran. }}\right)$ are a few of the checks completed in this step. These criteria do not relate to factored loads and are therefore evaluated for the total loading 
without load factors applied. As the first two steps are completed and the models meet the requirements for each, the structure is deemed redundant. Finally, the connections, especially those in the redundant bracing system, are all to be checked again to ensure there is sufficient capacity to carry the redundant loadings.

\subsubsection{Additional Approaches for Redundancy Evaluation}

Frangopol and Curley (1987) present various mathematical expressions to quantify the level of redundancy in highway bridges. These measures, similar to system reserve ratios discussed in Section 2.5.1, are simply functions of the capacity of a bridge under its intact state (denoted $\mathrm{L}_{\text {intact }}$ in this research), its capacity under a damaged state (denoted $\mathrm{L}_{\text {damaged}}$ ), and its design capacity $\left(\mathrm{L}_{\text {design }}\right)$. Specifically, these ratios, or "redundant factors," are as follows:

- $\quad$ Reserve redundant factor $=\mathrm{L}_{\text {intact }} / \mathrm{L}_{\text {design }}$

- $\quad$ Residual redundant factor $=\mathrm{L}_{\text {damaged }} / \mathrm{L}_{\text {intact }}$

- Strength redundant factor $=\mathrm{L}_{\text {intact }} /\left(\mathrm{L}_{\text {intact }}-\mathrm{L}_{\text {damaged }}\right)$

In addition to presenting these simple factors to express redundancy, the authors also present a simplified method to determine system-level redundancy:

1. Using plastic methods of structural analysis (or possibly other tools such as finite element analysis), determine the load at which system or partial system collapse occurs (or $\mathrm{L}_{\text {intact}}$ )

2. Next, determine a multiplier factor, $\lambda$, which relates the applied load on the bridge to the force effect present in a given member.

- $\lambda=\mathrm{L}_{\text {intact }} / \mathrm{L}_{\text {applied }}$, where $\mathrm{L}_{\text {applied }}=$ the applied load on the bridge

3. Next, assume that member has undergone some form of damage in which its damaged capacity is directly proportional to its undamaged capacity.

- For instance, this could be expressed as a damage factor, $\mathrm{D}_{\mathrm{g}}=\mathrm{M}_{\text {girder }}$ / $\mathrm{M}_{\text {girder, }}$ where $\mathrm{M}_{\text {girder }}$ would represent the plastic moment capacity of the undamaged girder and $\mathrm{M}_{\text {girder }}^{*}$ would represent the plastic moment capacity of the damaged girder.

4. Determine a new collapse load under the damaged state $\left(\mathrm{L}_{\text {damaged }}\right)$ 
5. Determine a new multiplier factor, $\lambda^{*}$.

- $\lambda^{*}=\mathrm{L}_{\text {damaged }} / \mathrm{L}_{\text {applied }}$

6. Finally, a system redundancy factor, $R$, can be calculated using $R=\lambda /\left(\lambda-\lambda^{*}\right)$

The value "R" would, in turn, be the systems measure of reserve strength between the damaged state and the collapse of the system. Its range would be from $R=1$ (i.e. the system has no reserve capacity at all, or $\lambda^{*}=0$ ) to $\mathrm{R}=\infty$ (i.e. the damaged component has no influence on system capacity at all, or $\lambda^{*}=\lambda$ ). With the advantages of finite element techniques, this method can prove useful in determining the effect that damaged components have on system-level reserve capacity as well as recommended inspection intervals. However, it should be noted that in some situations, some system components, such as secondary-level bracing, may not carry significant loads in the system's intact state but, in turn, may contribute considerably to the system's damaged capacity. Therefore, the authors suggest that the engineer evaluates how different structural components would transmit loads in damaged conditions.

In addition, the Task Committee on Redundancy (1985) gives recommendations for the analysis of bridges as it relates to structural redundancy. While these recommendations are not a specific method for determining redundancy, they can greatly assist computational efforts in quantifying redundancy. Specifically, they regard the following parameters as they relate to global bridge behavior:

1. It is suggested that, while skew may affect certain parameters such as live load distribution, overall bridge superstructure behavior is not greatly affected if the skew angle is less than $30^{\circ}\left(0^{\circ}\right.$ being indicative of a right bridge). It should be noted that this statement is meant to reflect behavior of slab-on-beam bridge systems.

2. Also, a critical bridge component that should be included in all modeling efforts is the presence of cross-frames or diaphragms. It is stated that during damaged conditions, diaphragms and lateral bracing elements contribute significantly to the integrity of the structure in load redistribution.

3. It is indicated that, in composite bridge construction, the first component to experience nonlinear material behavior will be the concrete deck. An example of this might be deck cracking over the interior supports in continuous bridges. In addition, as shown in studies by Hovell (2007), it has been shown that the 
nonlinear capacity of the deck contributes significantly to the load redistribution capabilities of a bridge in a damaged state.

4. The report does offer some caution as to the incorporation of material or geometric nonlinearity in an analysis model as they can increase the time necessary to perform an analysis. As great advances in computational efficiency have been made since this publication, the importance of computing time is somewhat less important. However, an important note that is recognized by the Task Committee on Redundancy regards small displacement theory vs. large displacement theory; if many load cases are considered, this can exponentially increase as the principle of superposition will no longer be valid (i.e. increasing the number of incremental analyses performed).

5. When analyzing details in bridge superstructures, it is recommended that area with stress concentrations be given due consideration. Specifically, secondary bridge elements, which may not contribute much to the intact structure, may be critical to the damaged system. As stated by the authors, "the risks of using elastic analysis techniques in highly strained regions cannot be overemphasized." In addition, the authors recommend that the analyst give consideration to displacement-induced stresses (such as out-of-plane deformation of the web of steel girders).

6. Finally, stability in flexural systems is not considered to be a major concern.

However, this statement concerns straight girder systems; for curved girder systems, the authors state that this is a significant interest and can present unique difficulties in determining global bridge behavior.

\subsection{CONCLUSIONS}

This chapter summarized various research projects and specifications regarding system redundancy in highway bridges. It was shown that, while many varying definitions exist regarding the meaning of redundancy, there are also common trends as to what should be considered when quantifying redundancy. For example, numerous studies included nonlinear analyses, similar limit states and similar loading effects to be applied to a bridge to determine the 
level of redundancy present. Therefore, a general set of procedures for evaluating a structure for redundancy can be developed based on various studies described in this chapter; this proposed set of procedures for determining the level of redundancy in a structure are outlined in further detail in the following chapter. 


\section{Chapter 3: DeVelopment of Protocols for the ACCURATe ASSESSment of Primary AND SECONDARy LoAd Path REDUNDANCY}

\subsection{INTRODUCTION}

Following the extensive background research performed in Chapter 2, it is necessary to develop a set of procedures with which to accurately assess the levels of redundancy present in a bridge. The methodology employed in this research is derived from the method presented in the National Cooperative Highway Research Program's (NCHRP) Report 406 (1997) and is discussed in the following chapter. In addition, particular attention is paid to the finite element modeling techniques used to capture the behavior of steel truss bridges.

\subsection{Finite Element Modeling TeChNiQues}

To quantify the levels of redundancy present in a structure, a finite element model is created using the commercial software package, Abaqus/CAE Version 6.10 (Dassault Systèmes, 2010). Element selection for these finite element models included a 4-node, doubly-curved, finite-membrane-strain, general-purpose shell with reduced integration (known in the Abaqus/Standard User's Manual as an S4R element) and a 2-node linear beam in space (known in the Abaqus/Standard User's Manual as a B31 element). S4R elements were used to simulate all structural elements with the exception of lateral rods, which were simulated with lateral rods. To model the composite action between both the deck and the stringers as well as all gusset plate connections, node-to-node multiple point constraints were used such that the degrees of freedom between nodes were restrained (these constraints are known in the Abaqus/Standard User's Manual as an MPC Beam). In addition, for all the finite element models presented in this study, the boundary conditions are all representative of pinned-roller connections.

The constitutive model used to characterize the mechanical behavior of Grade 50 steel in this study consists of a multi-linear approximation (Galindez, 2009), which is shown in Figure 
3.1. A representative value for the modulus of elasticity ( $E=29600 \mathrm{ksi}$ ) was specified to construct the curve. Finally, the engineering stress-strain relationship is converted to the true stress-strain curve, as required for input into Abaqus/Standard.

In lieu of performing a mesh density study, an approximate element size of 1 " $\times 1$ " was used for all main load carrying elements. Studies by Yang (2004) found that inelastic behavior of steel members can be accurately observed when finite element meshes are composed of S4R shell elements approximately this size.

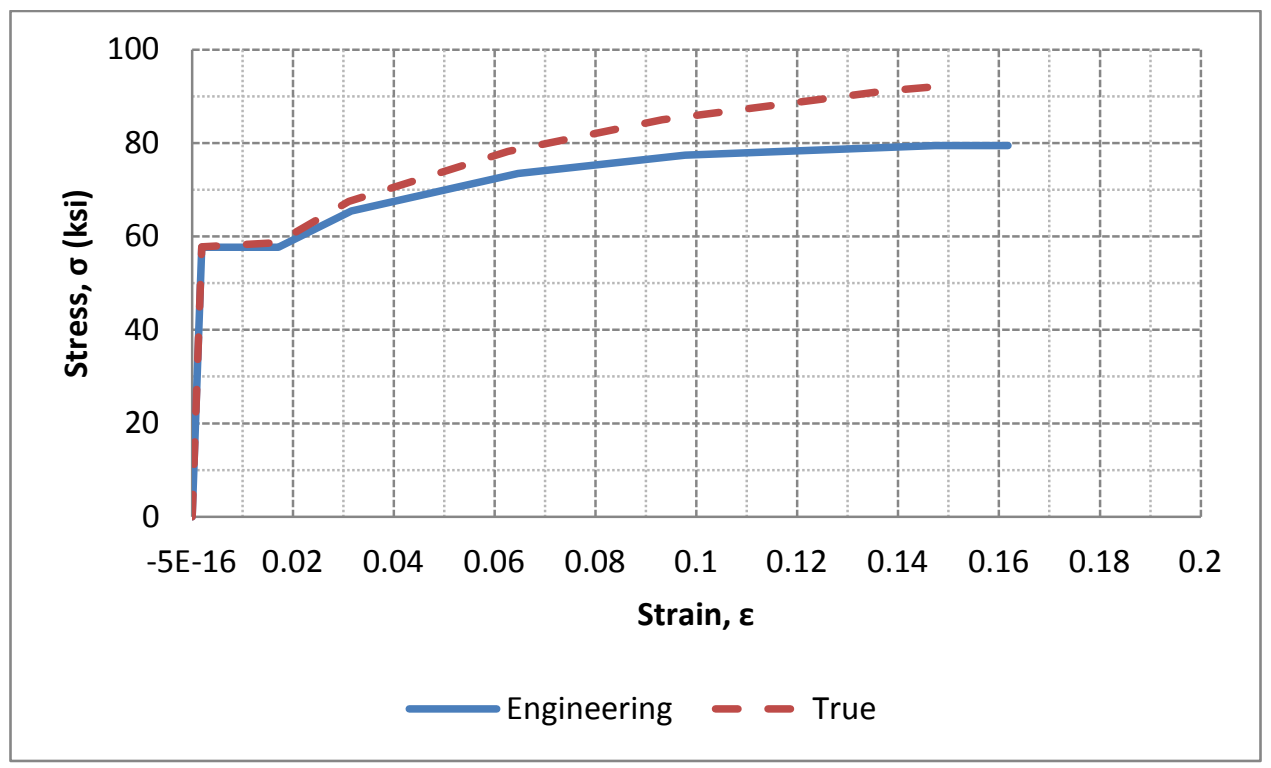

Figure 3.1: Steel Stress-Strain Relationship (Galindez, 2009) 


\begin{tabular}{ccc}
\hline Point & Strain & Stress \\
\hline 1 & $\frac{\sigma_{s y}}{E}$ & $\sigma_{s y}$ \\
\hline 2 & $\varepsilon_{s t}$ & $\sigma_{s y}$ \\
\hline 3 & $\frac{\left(\varepsilon_{u}-\varepsilon_{s t}\right)}{10}+\varepsilon_{s t}$ & $\frac{E_{s t}\left(\varepsilon_{u}-\varepsilon_{s t}\right)}{10}+\sigma_{s y}$ \\
\hline 4 & $\frac{2\left(\varepsilon_{6}-\varepsilon_{3}\right)}{7}+\varepsilon_{3}$ & $\frac{4\left(\sigma_{6}-\sigma_{3}\right)}{7}+\sigma_{3}$ \\
\hline 5 & $\frac{2\left(\varepsilon_{6}-\varepsilon_{3}\right)}{7}+\varepsilon_{4}$ & $\frac{2\left(\sigma_{6}-\sigma_{3}\right)}{7}+\sigma_{4}$ \\
\hline 6 & $\varepsilon_{u}-\frac{\left(\varepsilon_{u}-\varepsilon_{s t}\right)}{10}$ & $\left(\frac{\sigma_{s y}}{\sigma_{0.2 \%}}\right) \sigma_{u}-\frac{100\left(\varepsilon_{u}-\varepsilon_{s t}\right)}{E_{s t}}$ \\
\hline 7 & $\varepsilon_{u}$ & $\left(\frac{\sigma_{s y}}{\sigma_{0.2 \%}}\right) \sigma_{u}$ \\
\hline
\end{tabular}

\begin{tabular}{lc}
\hline \multicolumn{1}{c}{ Property } & Average Value \\
\hline Static yield strength, $\sigma_{s y}(\mathrm{Ksi})$ & 57.65 \\
\hline Offset yield strength, $\sigma_{0.2 \%}(\mathrm{Ksi})$ & 59.42 \\
\hline Strain at the onset of strain hardening, $\varepsilon_{s t}(\%)$ & 1.71 \\
\hline Strain hardening modulus, $E_{s t}(\mathrm{Ksi})$ & 537.37 \\
\hline Tensile strength, $\sigma_{u}(\mathrm{Ksi})$ & 81.88 \\
\hline Strain at the tensile strength, $\varepsilon_{u}(\%)$ & 16.18 \\
\hline
\end{tabular}

Figure 3.2: Multi-linear Stress-Strain Approximations (Galindez, 2009) 
The vehicular live load used to assess redundancy throughout this study is the AASHTO HL-93 design truck (AASHTO, 2010). Figure 3.3 shows a side and back view of the HL-93 truck. It should be noted that, while it is the engineer's responsibility to vary the rear-axle spacing to produce maximum force effects, the rear axle spacing was kept constant at $14 \mathrm{ft}$ as this will produce maximum force effects in simple-span structures.

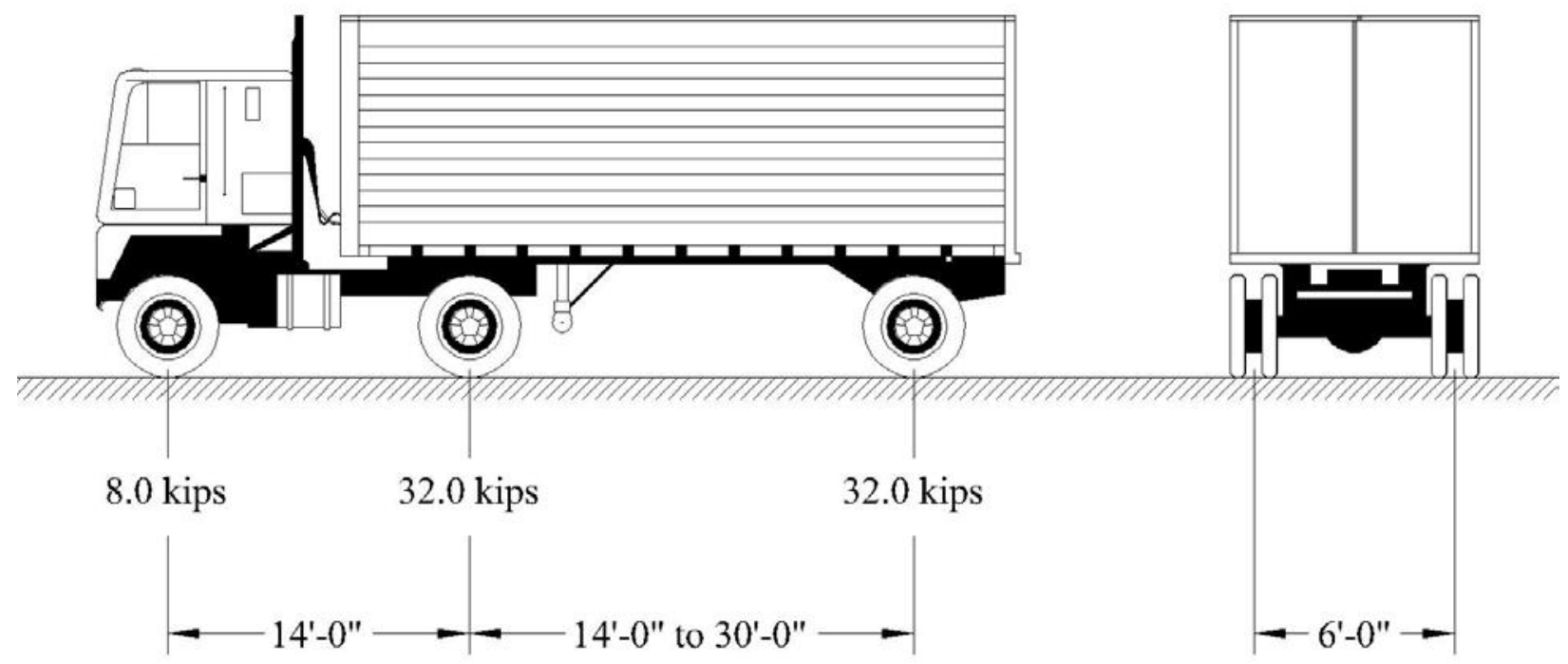

Figure 3.3: AASHTO HL-93 Design Truck (AASHTO, 2010)

According to AASHTO Section 3.6.1.2.5, the tire contact area of a given wheel (regardless of whether it consists of one or two wheels) should be assumed to act of a 10 in. by 20 in. rectangle. Therefore, the vehicular live loads were applied to the finite element models by using equivalent pressure loads applied over a 200-square-inch area on the deck mesh. The longitudinal and transverse placement of this truck (and the number of trucks placed) will be discussed in Section 3.4.

\subsection{DEFINITION OF LiMit StATES}

Following a thorough examination of the various limit states presented in Chapter 2, two limit states were chosen to be included in the proposed redundancy analysis procedures: 
individual member failure and damaged condition capacity. Each limit state is further described in Sections 3.3.1 and 3.3.2.

\subsubsection{Member Failure}

This limit state is in place to ensure proper member capacity as specified in the current AASHTO specifications. A linear elastic model of the bridge is analyzed for the capacity of the structure to resist first member failure (Ghosn and Moses, 1997). Dead loads (i.e. the self-weight of the system) are applied along with an incremental live loading of two AASHTO HL-93 vehicles. These vehicles are incrementally augmented until first member failure is reached. An HL-93 load multiplier, $\mathrm{LF}_{1}$, can then be calculated which represents the scaled magnitude of the two trucks over the original magnitude.

\subsubsection{Damaged Conditions}

To effectively determine the redundancy of a bridge, several levels of damaged conditions must be evaluated. Specifically, analysis must be performed on a nonlinear structural model after the removal of a main member or load-carrying component. This member could be a previously identified fracture-critical member or a member that is susceptible to other types of damage, such as corrosion or collisions by vehicles or boat traffic. The loads described in Section 3.3.1 are again applied incrementally; however, the structure is loaded until collapse rather than a specified limit. In addition, the load multiplier for this limit state is $\mathrm{LF}_{\mathrm{d}}$ which represents the HL-93 multiplier at which the bridge collapses.

\subsection{Proposed Procedure for Redundancy Evaluation}

The assessment of the capacity of a bridge in a damaged state is the primary goal of the proposed methodology. Specifically, the methodology used in this project, much like the protocols specified in NCHRP Report 406 (Ghosn \& Moses, 1997) to assess load-path redundancy in a given bridge, is as follows: 
1. Determine individual member capacities according to current specifications. In this research, this was done by employing the Truss Rating and Analysis Program (TRAP) developed by the BEST Center at the University of Maryland (2010).

2. Develop a finite element model of the system which simulates all main load-carrying components and is capable of both linear and nonlinear analysis. For a description of the modeling procedures used in this research, see Section 3.2.

3. Apply the dead load (i.e. self-weight) and live loads to the structure. For live loading, two HL-93 design trucks (see Section 3.3) are placed side-by-side and positioned both longitudinally and transversely to produce maximum force effects.

4. Determine which main load-carrying members are fracture-critical or whose failure may have the most impact on the overall structure. It should be noted that fracture-critical members are defined by AASHTO (2010) as "components in tension whose failure is expected to result in the collapse of the bridge or the inability of the bridge to perform its function."

5. Perform a linear elastic analysis of the structure. For this analysis, the dead load is applied first, then the magnitudes of the live loads are incrementally augmented until the capacity of a given member is reached. The multiplier of the live loads (termed $\mathrm{LF}_{1}$ ) that caused the member capacity to be reached is then recorded.

6. Simulate damaged conditions in one of the chosen fracture-critical members by performing a cut-chord analysis. The member in question is severed such that it provides no structural resistance to the system.

7. Perform a nonlinear analysis of the damaged structure (considering both geometric nonlinearities and the nonlinear material properties specified in Section 3.2). The dead load is applied first, and then the magnitudes of the live loads are incrementally augmented until collapse of the system is reached. The resulting multiplier of the live loads (termed $\mathrm{LF}_{\mathrm{d}}$ ) is then recorded.

8. Calculate the redundancy ratio for the member in question. Specifically, the damaged condition ratio, $\mathrm{r}_{\mathrm{d}}$, is the ratio of $\mathrm{LF}_{\mathrm{d}}$ found in step 7 and $\mathrm{LF}_{1}$ determined in step 5 .

9. Repeat steps 7 through 9 for all members in the system identified to be fracture-critical.

10. Compare these redundancy ratios to previously defined limits. For this research, the limits specified in NCHRP Report 406 are utilized. Therefore, according the NCHRP 
Report 406, if the value of $r_{d}$ is greater than or equal to 0.50 , the bridge is deemed to possess adequate levels of load-path redundancy.

\subsection{CONCLUSION}

The redundancy analysis procedures outlined in this chapter were employed in Chapter 5 on a representative steel truss bridge. The process proposed is very similar to the procedure specified in NCHRP Report 406. However, an accurate finite element modeling technique is critical in order to ensure accurate results of the redundancy analysis. To verify the validity of the modeling approach used in this effort, a field test was performed on a steel truss bridge in Jackson County, West Virginia. This field test, as well as a comparison between experimental and analytical results, is discussed in the following chapter. Once the modeling technique has been demonstrated to be sufficiently accurate to predict the behavior of steel truss bridges (discussed in Chapter 4), it can be used in conjunction with the proposed protocols to determine the level of redundancy present in these types of bridges (discussed in Chapter 5). 


\section{Chapter 4: LitTle Mill Creek Bridge Field Test}

\subsection{INTRODUCTION}

To verify the validity of the finite element modeling technique presented in the previous chapter, a physical load test was performed on the Little Mill Creek Bridge in Jackson County, West Virginia on November 7, 2011. Data from the field testing of the Little Mill Creek Bridge were compared against the results of a finite element model of the bridge using the previously described modeling technique.

Contained in this chapter is an overview of the physical investigation of the Little Mill Creek Bridge. A brief description of the bridge is provided along with an overview of the field test procedure and equipment used. In addition, a comparison of the experimental data and data obtained from a finite element model developed using the methods discussed in Chapter 3 is presented.

\subsection{BRIDGE DESCRIPTION}

The bridge chosen for the field investigation in this study is a 150 foot span, two-lane steel truss system located in Jackson County, West Virginia. Carrying local traffic over Little Mill Creek on County Route 5/18, the bridge is comprised of wide flange sections and miscellaneous channels. The through truss geometry consists of ten evenly spaced panel points. Wide-flange shapes are used for the top chords, diagonal members, and vertical members. For the bottom chords, two miscellaneous channels are used as a built-up section. In addition, wide flange shapes are used for the floor beams and stringers. The bridge carries two 12-ft. lanes of traffic on an asphalt surfaced corrugated steel deck. The bearings are 28 in. $\mathrm{x} 18$ in. steel soleplates with a total thickness of 3.5 in. For other specific dimensions, the reader is referred to the testing plan discussed in Section 4.4. An isometric view of the bridge is shown in Figure 4.1. 


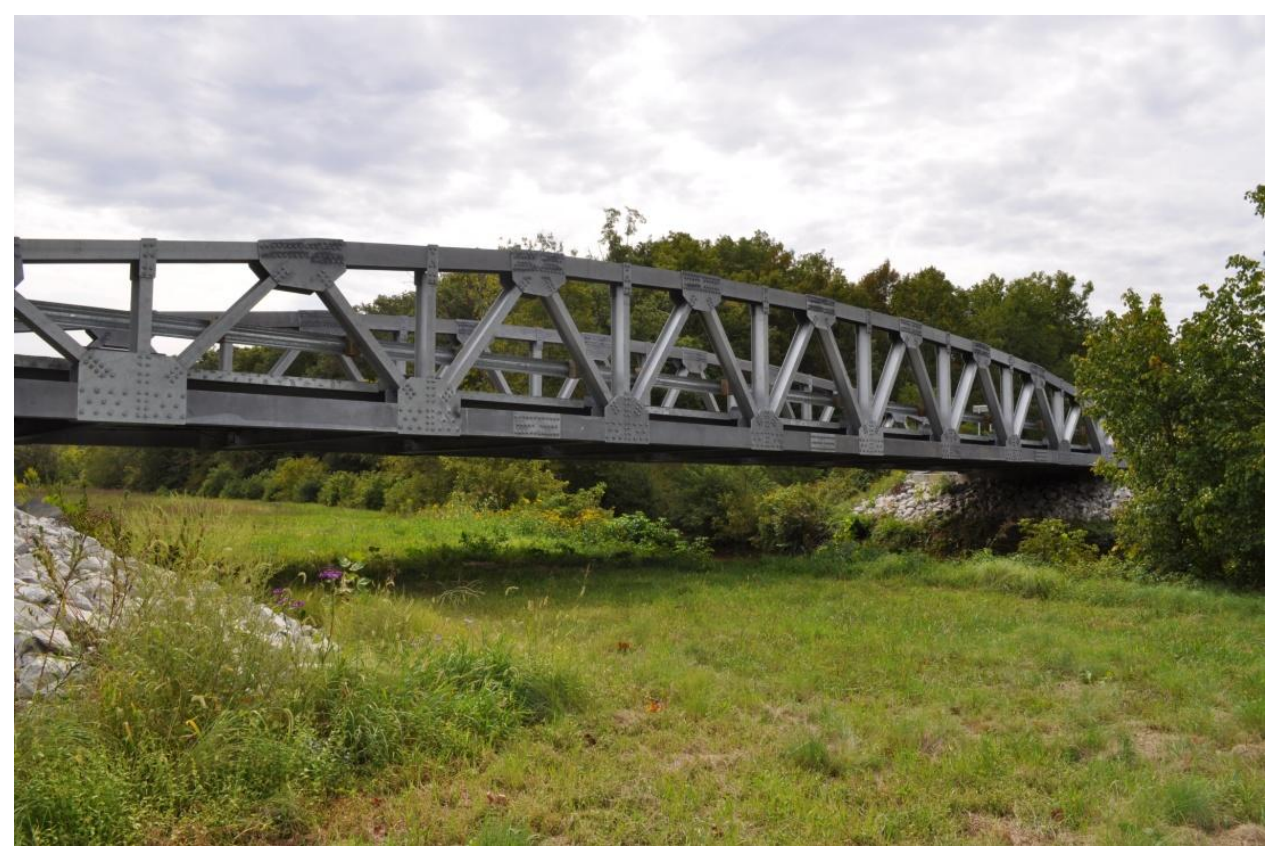

Figure 4.1: Isometric View of Little Mill Creek Bridge

\subsection{SUMMARY OF EQUIPMENT USED}

The following section contains an overview of the equipment used to perform the field investigation of the Little Mill Creek Bridge.

\subsubsection{STS-WiFi Data Acquisition System}

Strains and deflections were collected and recorded by a suite of wireless instruments, devices and software from Bridge Diagnostics, Incorporated (BDI). The BDI wireless system can accommodate several different types of instruments and incorporates 4-channel nodes and a wireless base station. Each instrument generally comes equipped with BDI's "Intelliducer" chip that allows it to identify itself within the software. This eliminates confusion during postprocessing when trying to distinguish between data collected by various gages. The instruments used during this field test were BDI strain transducers and RDP LVDTs (see Sections 4.3.2 and 4.3.3). 
The wireless base station shown in Figure 4.2 is used to monitor real-time wireless broadband signals that are transmitted over several hundred feet from the 4-channel nodes (shown in Figure 4.3). The nodes also monitor and power the instruments when online. The base station is capable of taking readings at 500 samples per second $(500 \mathrm{~Hz})$ and has an expandable channel capacity ranging from 4 to 128 channels; expandable in multiples of four.

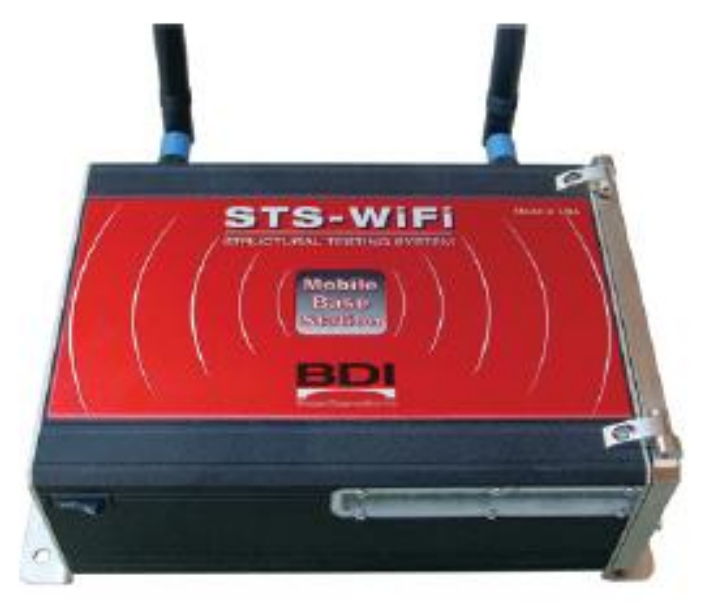

Figure 4.2: Wireless Base Station

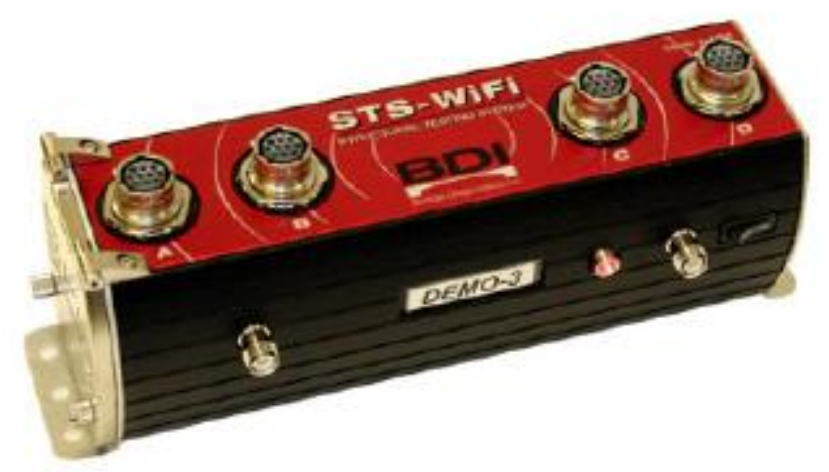

Figure 4.3: 4-Channel Node

This test system saves a lot of time because it requires no wiring between the base station and the instruments. The nodes and base station are powered by rechargeable 9.6V Makita NiMH batteries that can last up to six hours under continuous use. The BDI software also has a standby function that allows users to put all or some of the nodes to sleep for a given amount of 
time. This allows users to run tests all day on a single battery charge without having to spend valuable time retrieving the nodes to replace batteries.

\subsubsection{Strain Indicators}

The strain gages selected for the field test were BDI's re-usable strain transducers (Figure 4.4). They are ideal for field-testing because they require minimal surface preparation and take very little time to install. The gages have a temperature range of $-60^{\circ} \mathrm{F}$ to $+250^{\circ} \mathrm{F}$ and connect to the nodes with military style quick connect plugs requiring no solder. Each gage has a range of $\pm 2000 \mu \varepsilon$ with an accuracy of \pm 2 percent. Reusable mounting studs were glued to the bridge with an instant adhesive and mounted with a jig to ensure proper stud spacing. The jig also reduced the risk of damaging the gages while tightening the nuts. The mounting studs fit through two holes on either end of the gage and tightened with two 7/16-in. nuts. The recommended adhesive was Loctite 410 Black Toughened Adhesive. The gage locations were first marked using black permanent markers and then prepped with a hand grinder to remove galvanization and any rust present. The adhesive was then applied to the bottom of the transducer tabs and pressed against the member at the marked locations and held in place for approximately one minute until secure.

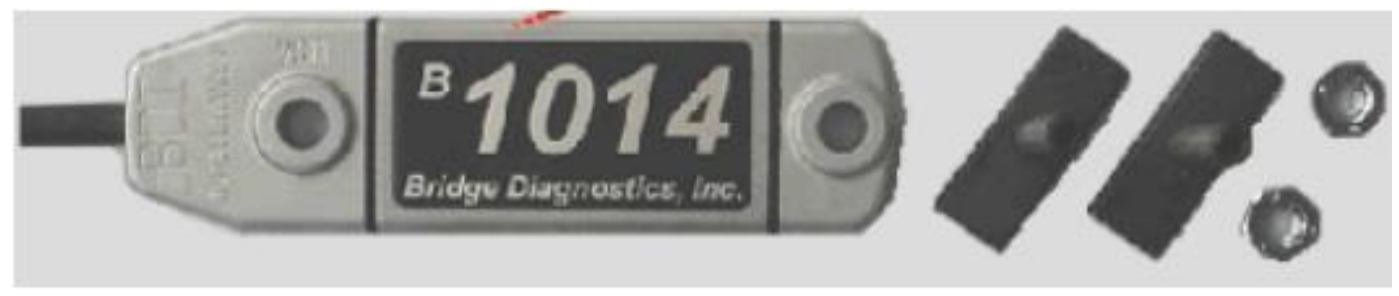

Figure 4.4: BDI Strain Transducer

\subsubsection{Linear Variable Differential Transducers (LVDTs)}

Deflections were simultaneously recorded at two consecutive panel points as well as at the center of two floor beams using 6 linear variable differential transducers (LVDTs) (see 
Section 4.4 for details regarding testing plan). The LVDTs were provided by BDI and manufactured by RDP Electronics Group, and are shown in Figure 4.5. They have a range of \pm 1 inch, an accuracy of $\pm 0.1 \%$, and an operating temperature range of $-58^{\circ} \mathrm{F}$ to $158^{\circ} \mathrm{F}$.

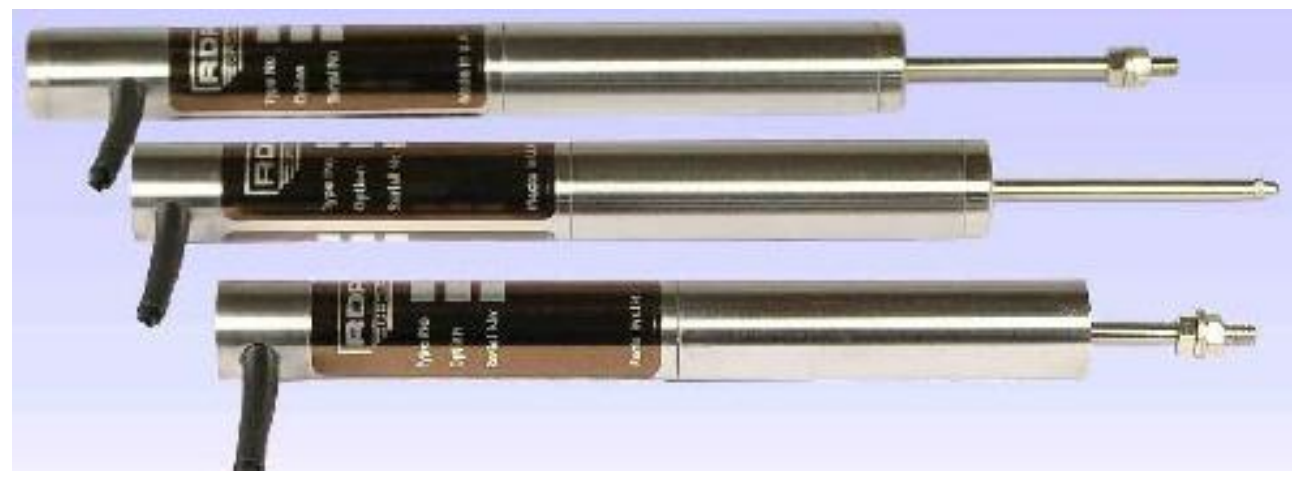

Figure 4.5: RDP Electronics Group LVDTs

\subsubsection{WVDOH Load Truck \& Wheel Scales}

A fully-loaded tandem-axle dump truck was provided by the West Virginia Department of Highways (WVDOH) to induce various structural behaviors. The truck was weighed and measured with the wheel-load scales (see Section 4.5.5) and a tape measure. The truck is shown in Figure 4.6 dimensions and wheel weights are shown in Figure 4.7. 


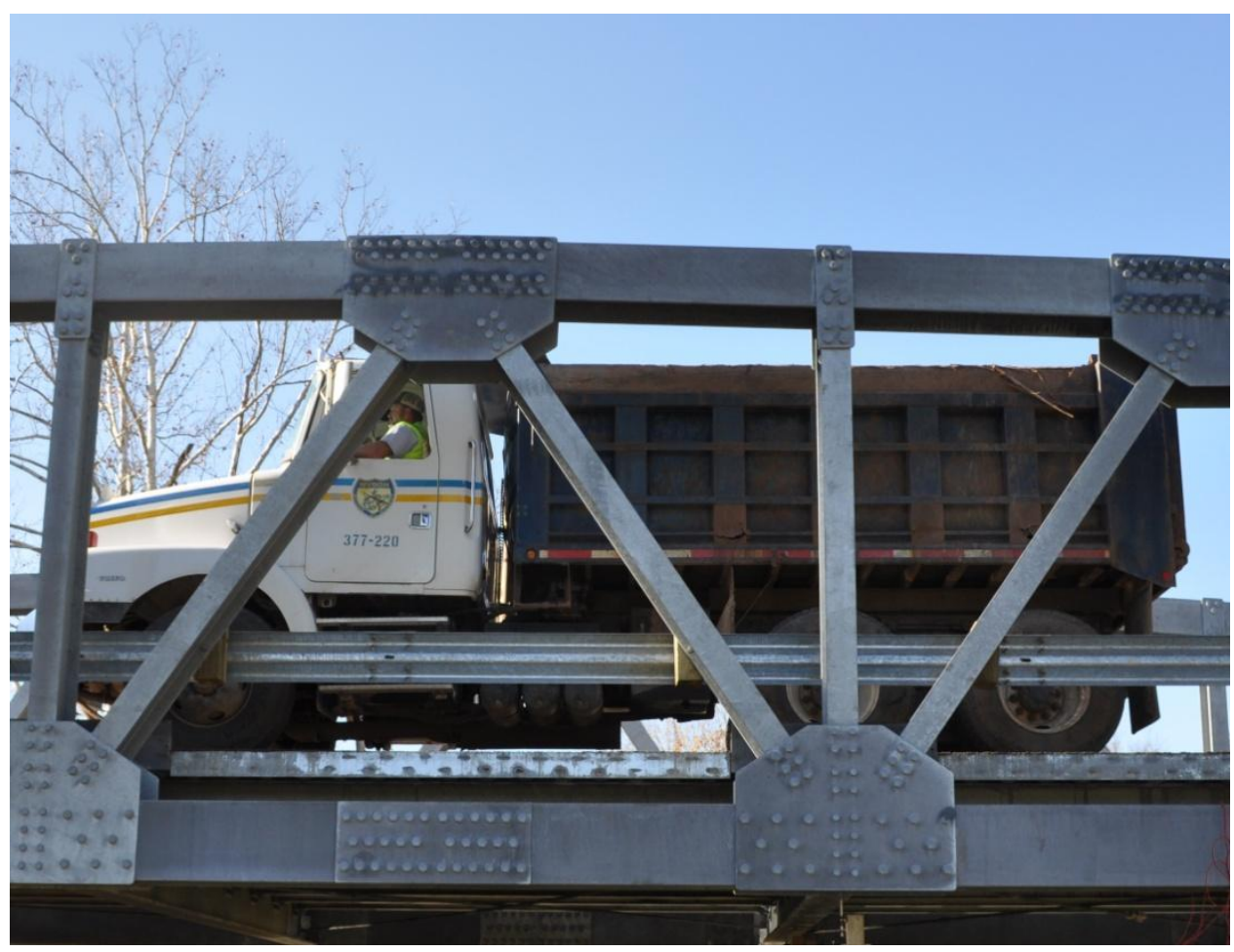

Figure 4.6: WVDOH Dual Tandem-Axle Load Truck

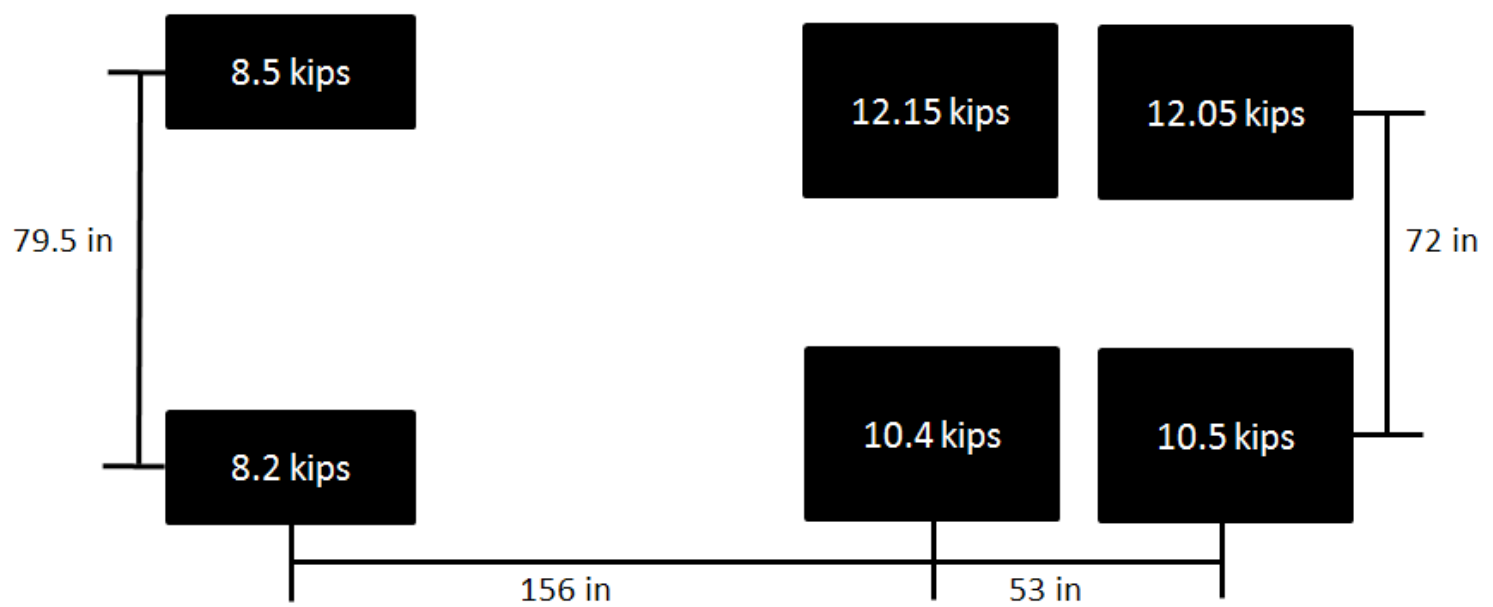

Figure 4.7: WV-DOH Dual Tandem-Axle Truck Wheel Loads

To weigh the load truck's wheels before beginning the load test, six Haenni portable wheel-load scales were provided by the West Virginia State Police on the day of field testing, and shown in Figure 4.8. 


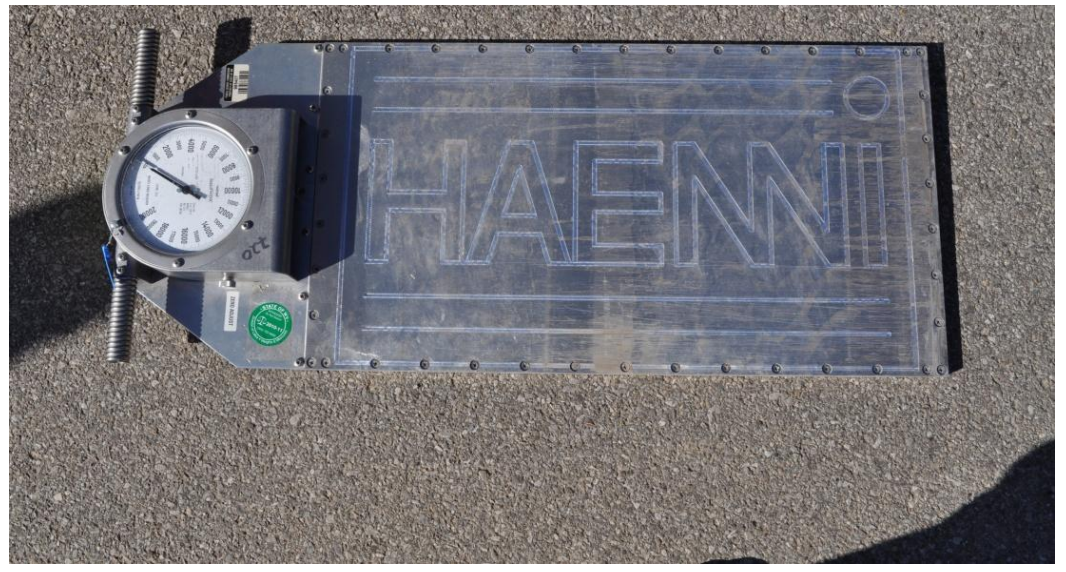

Figure 4.8: Wheel-Load Scale

\subsubsection{Miscellaneous Equipment}

The placement of the strain transducers required the use of several pieces of equipment listed below:

- extension cord

- square

- measuring tape

- portable grinder

- $7 / 16^{\prime \prime}$ socket set

The tools required to construct the LVDT frame were:

- post-hole digger

- saw

- power drill

- hammer

- screws/nails

- Handi-Crete mix

- bubble-level

- 4-in. by 4-in. posts 
- 2-in. by 4-in. posts

Additional equipment used for the field test included a four-wheel-drive articulated boom for use in safely instrumenting top chords and vertical members, a Dell laptop for use in conjunction with the data acquisition system, and a gasoline generator used in tandem with an APC battery backup and surge protection system for uninterrupted power supply.

\subsection{InSTRUMENTATION Plan}

The field test of the Little Mill Creek Bridge consisted of two days of preparation and one day of physical testing. During the first two days on location, several tasks were performed. The first task was to construct a rigid frame under the bridge to support the LVDTs (shown in Figure 4.9). The second task was to measure and mark out the locations for each strain gage and LVDT to be placed on the bridge for testing. This also included the necessary surface preparation for the BDI strain indicators (i.e. grinding away rust and galvanization). The third day was the day of the physical load test during which the strain gages were moved between two different setups. This was easily completed because the reusable strain gages were used in conjunction with a wireless data acquisition system (see Section 4.3.2).

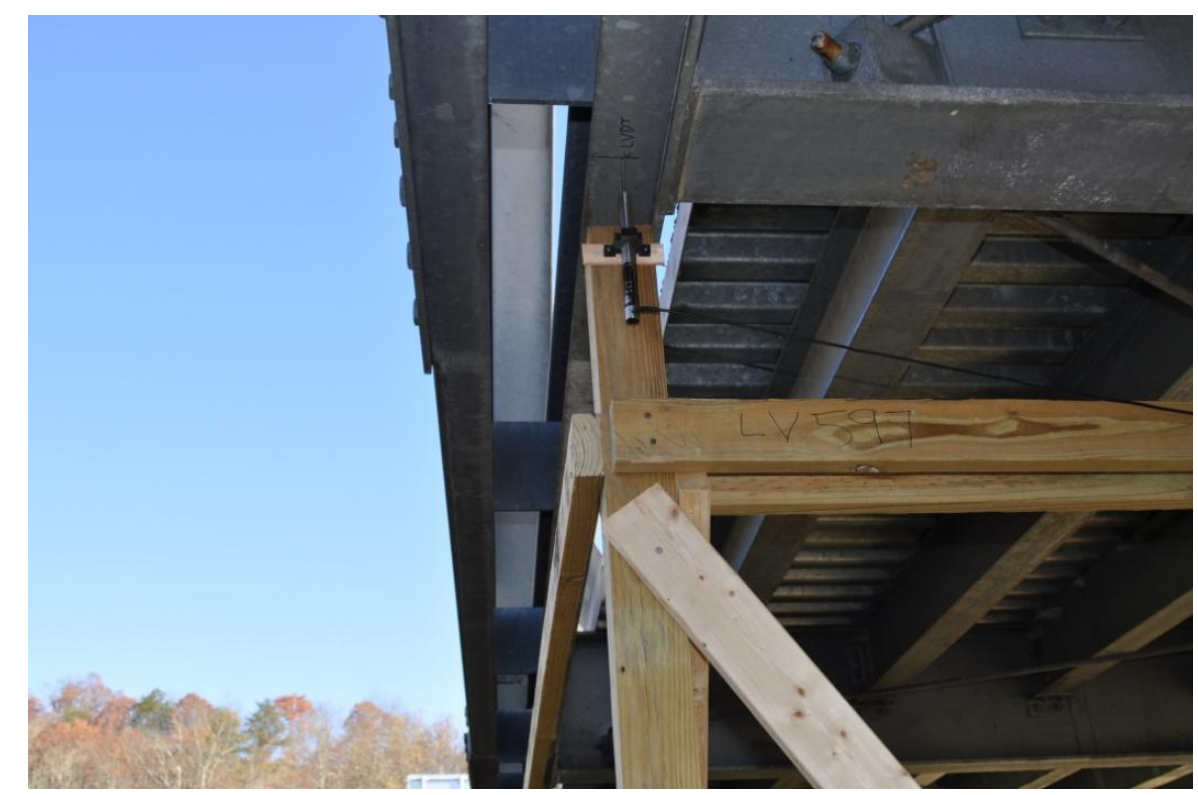

Figure 4.9: LVDT Stand 
In total, 28 independent gage locations and 6 independent LVDT locations were chosen for physical investigation. The location of these gages was primarily between the third and fourth panel points from the north abutment (this numbering refers to the panel point at the abutment as panel point zero). This was chosen as it was the set of panel points closest to the mid-span of the bridge that was accessible (due to the presence of the creek). Due to the symmetry of the bridge, instrumentation on truss chords could be concentrated on one truss line (for this field test, the east line of trusses was chosen for its proximity to a small access road).

Clusters of strain gages were placed on respective truss chords between panel points three and four. Multiple gages were employed in order to obtain redundant data from the field test as well as to capture any possible effects other than purely axial deformation (i.e. unintended bending of truss chords due to end rotations). The details regarding specific strain gage placement (as well as LVDT locations) are shown in Figures 4.10 through 4.13.

In summary, strain gages measuring axial strain were placed at the center of respective truss chords. Six gages were placed on the top chord, eight gages were placed on the bottom chord, four gages were placed on the diagonal member, and five gages were placed on the floor beams at both panel points three and four. Also, LVDTs measuring vertical displacements were placed on panel points on either side of the bridge at points three and four; in addition, LVDTs were placed at the mid-span of the floor beams on these respective panel points. 


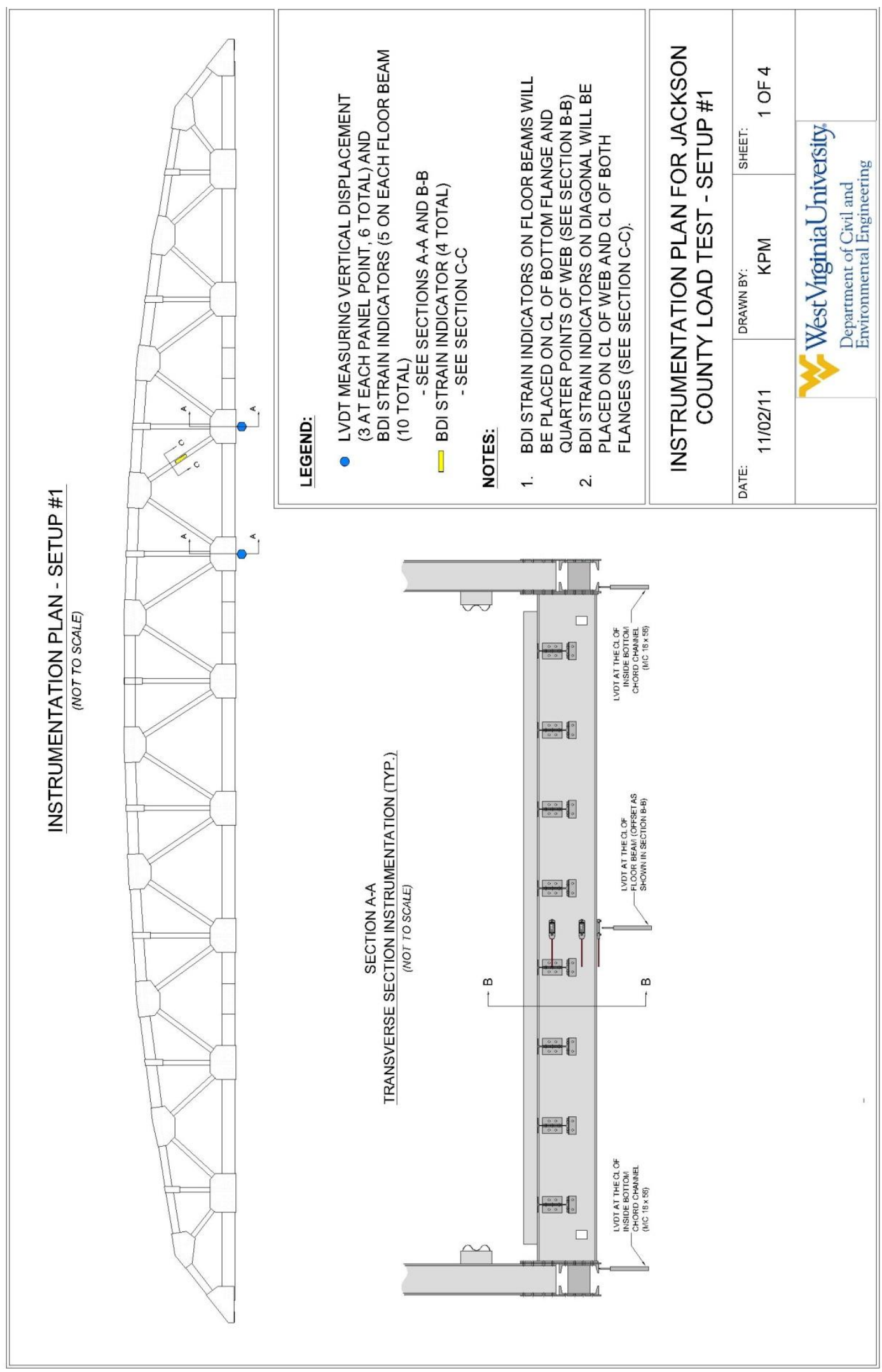

Figure 4.10: Instrumentation Plan (Sheet 1 of 4) 


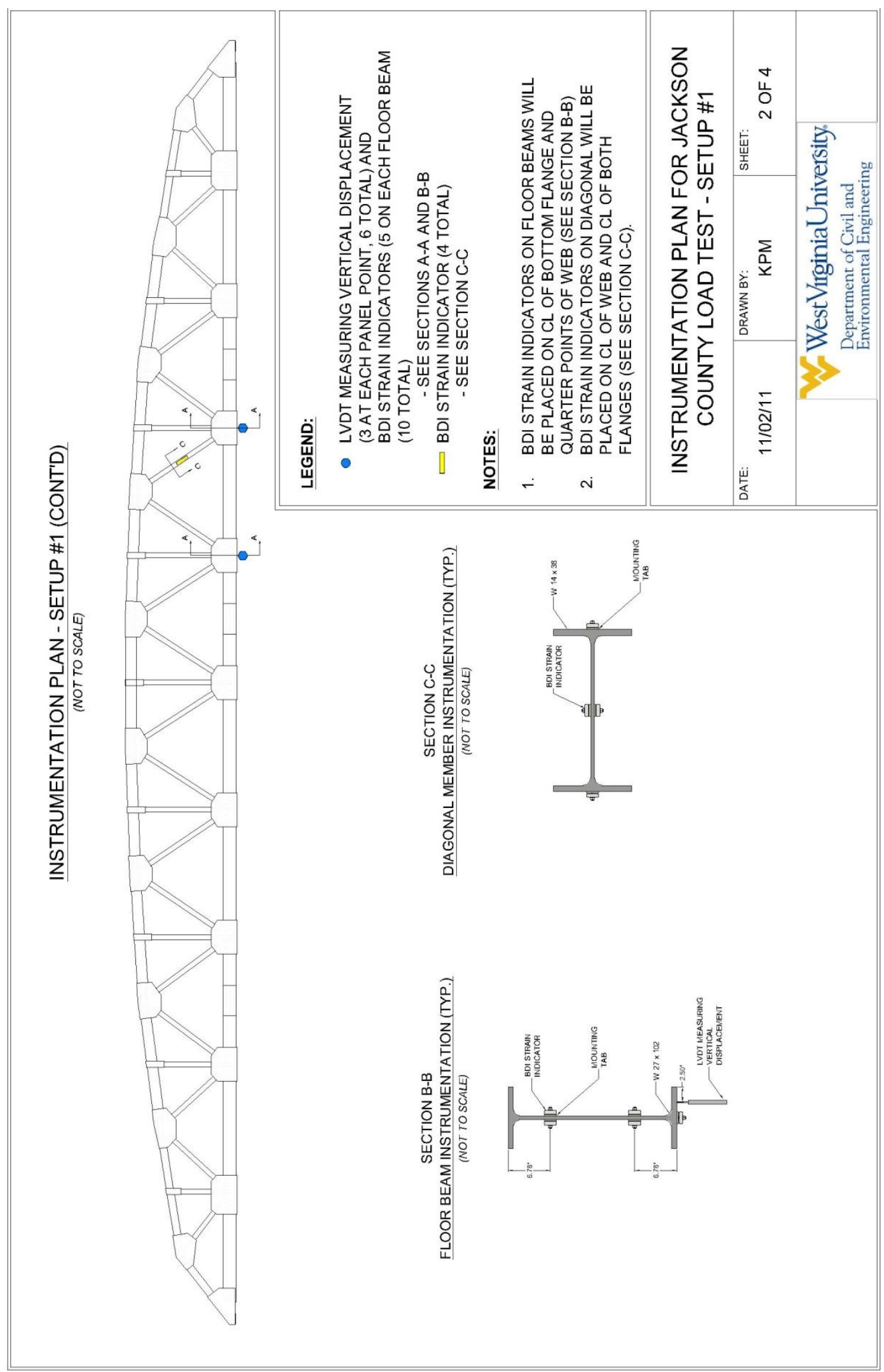

Figure 4.11: Instrumentation Plan (Sheet 2 of 4) 


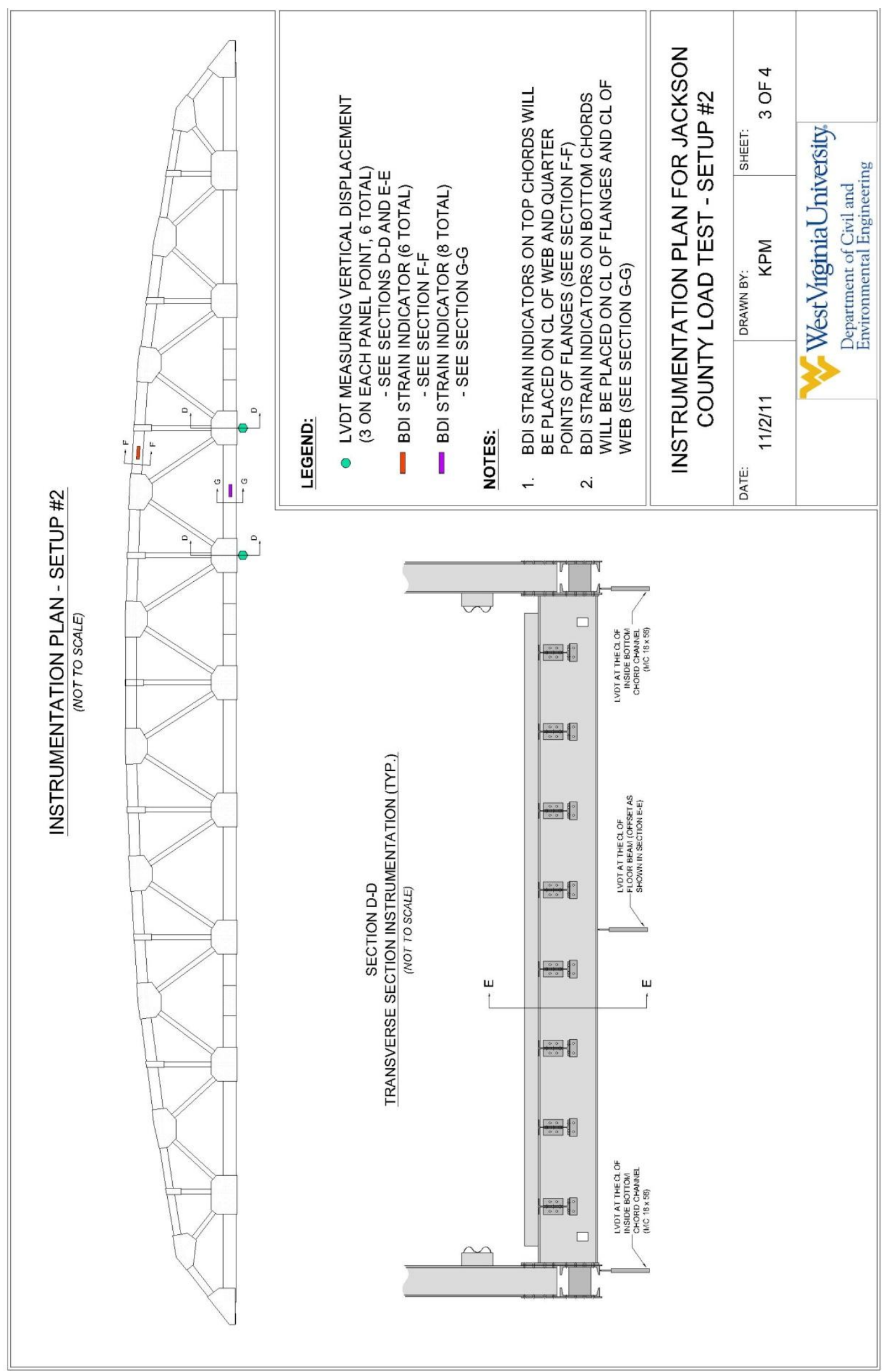

Figure 4.12: Instrumentation Plan (Sheet 3 of 4) 


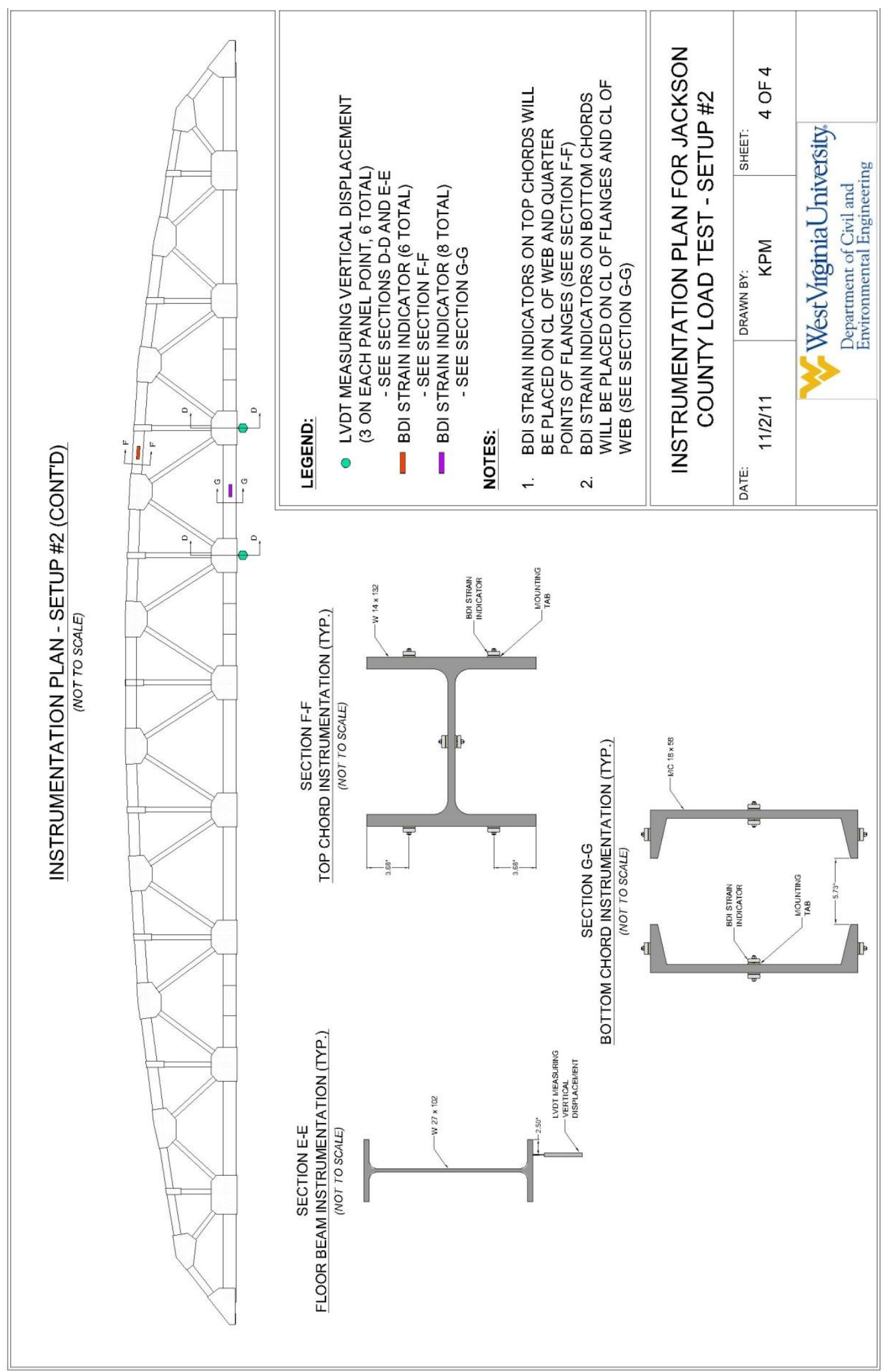

Figure 4.13: Instrumentation Plan (Sheet 4 of 4) 
Careful consideration was placed on determining which members were to be instrumented for the load test. The main members between the third and fourth panel points were instrumented in two setups. The first setup focused on measuring strains in both the diagonal chord and floor beams three and four where as the second setup focused on measuring strains in the top and bottom chords. In addition, the LVDTs measuring vertical displacement remained throughout both setups. This was done so displacements between setups could be monitored; in other words, if the load truck was positioned on the same location on the bridge for both setups, the LVDTs should theoretically yield the same displacement readings.

Two truck placements were used for each setup: a 2 foot offset from the guardrail and the centerline of the bridge. To maximize the effect of the vehicular live load on a particular line of truss, according to AASHTO Section 3.6.1.3.1, the design truck shall be placed such that the center of the wheel is no closer than 2 feet from the edge of the design lane.

For each truck run, the truck was driven at a crawling speed, and then stopped with the center axle resting over each respective panel point. The resulting racking and induced vibrations were allowed to settle to obtain a "pseudo-static" reading from each gage. This is desirable both from a design standpoint (current design methods use static analyses to obtain live load envelopes) and from a modeling standpoint (the proposed modeling technique assumed static behavior). Figure 4.14 shows the truck placed over a given panel point during the field test (in this case, panel point three). 


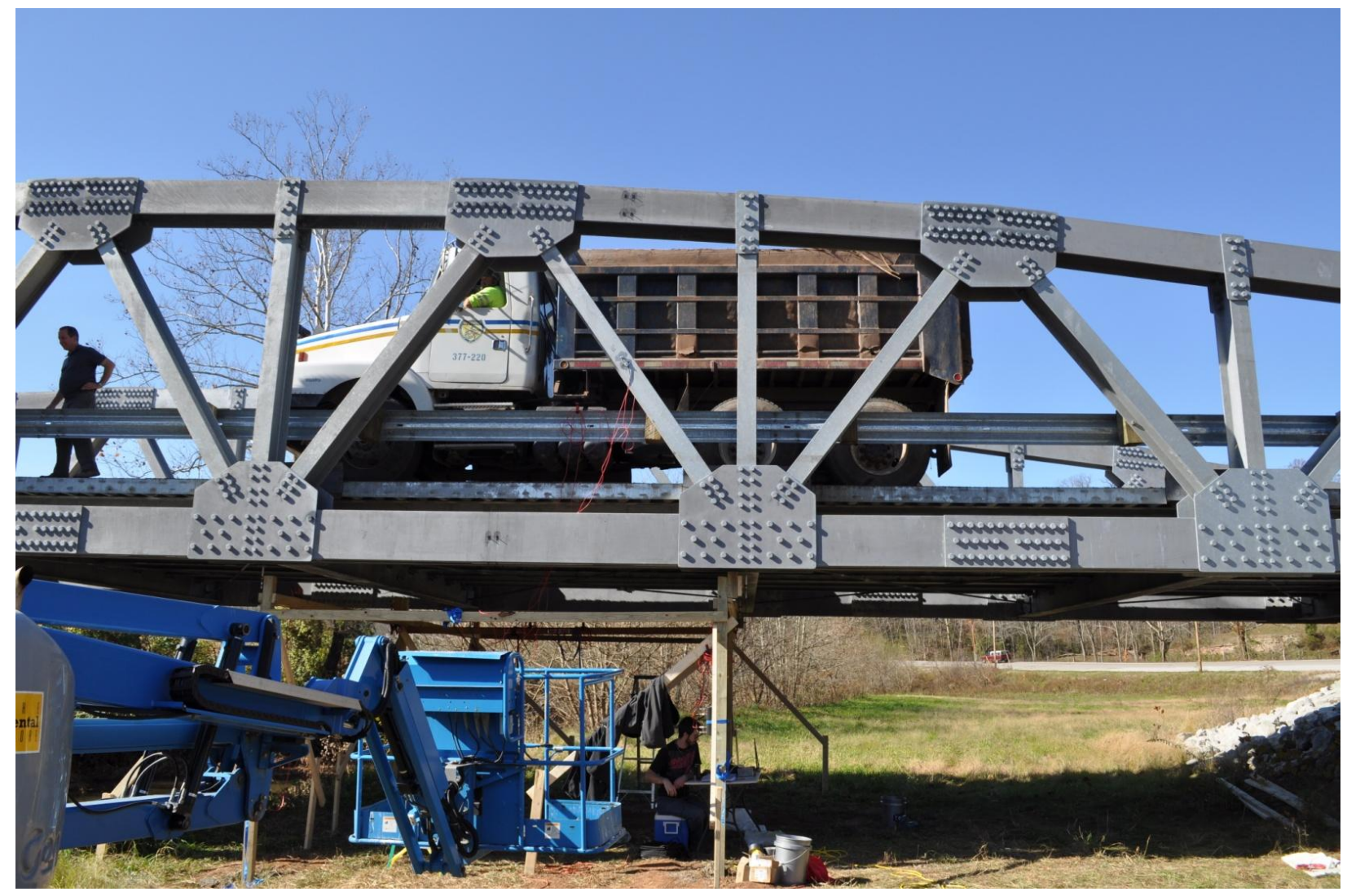

Figure 4.14: Load Truck Over Panel Point during Field Test

\subsection{Finite ELEMENT MODEL}

The finite element model of the Jackson County bridge was created in Abaqus/CAE Version 6.10 (Dassault Systèmes, 2010) using the finite element modeling technique as described in Section 3.2. The following images are screen captures of the finite element model: an intact view along with a close-up of a representative top chord gusset plate view to present the detailed mesh which was used. 


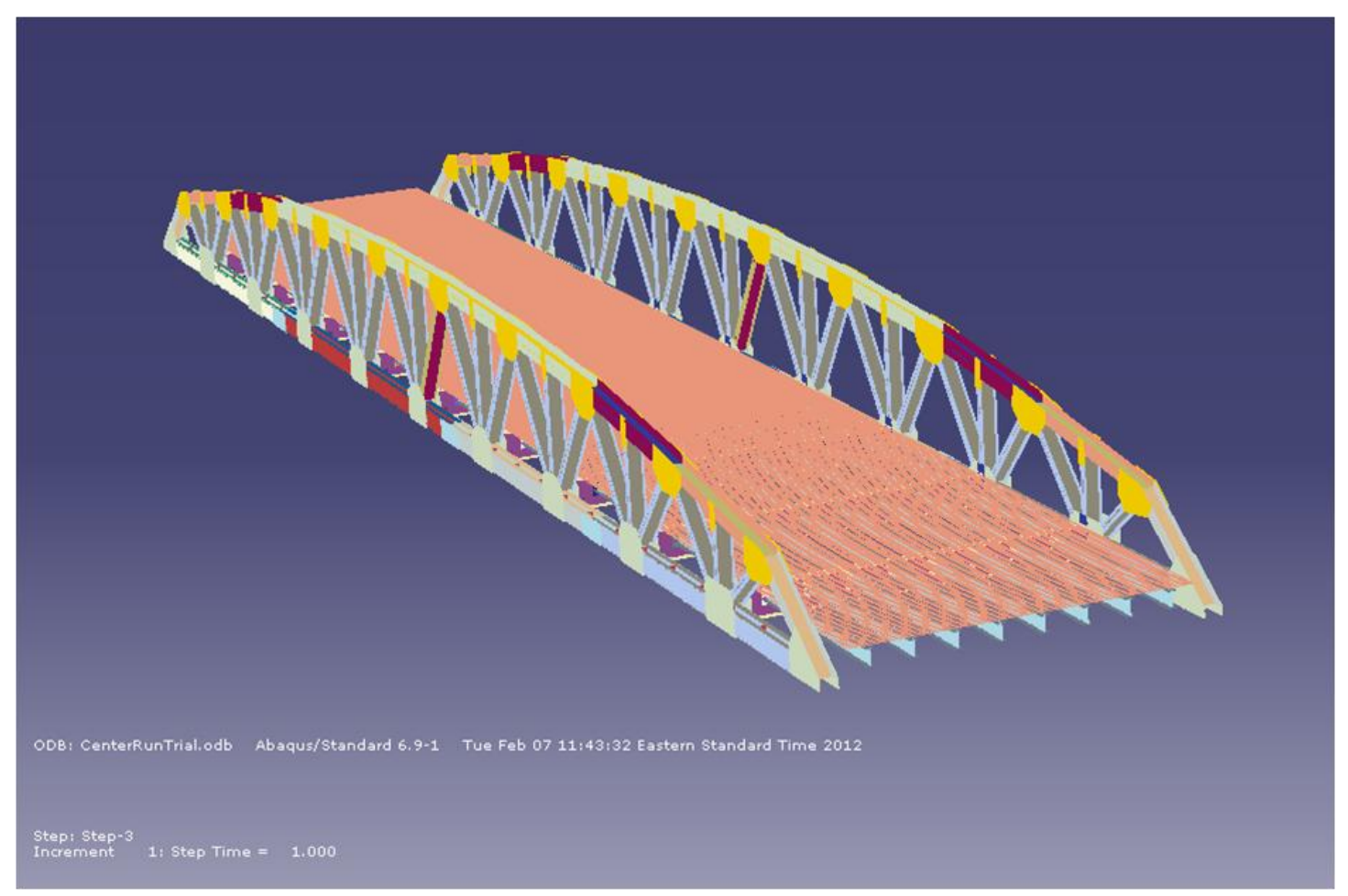

Figure 4.15: Intact Finite Element Model Screen Capture

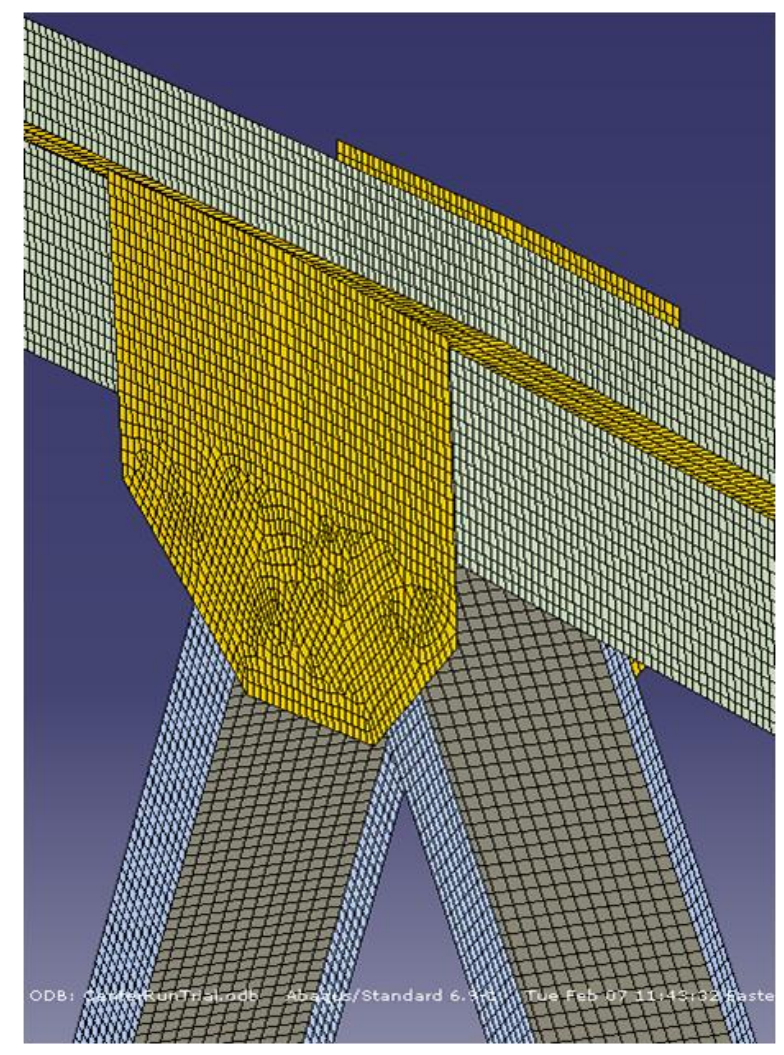

Figure 4.16: Top Chord Gusset Plate View 


\subsection{RESUlts AND COMPARISON WITH FINITE ELEMENT MODELING}

Following the field test, the finite element model created as shown in Section 4.5 was validated by comparing the results from the field test. This section includes a representative sample of graphs comparing the field test data with the corresponding finite element model data. The data correlated well indicating strong accuracy in the finite element modeling technique employed. For a more detailed comparison, the reader is referred to the Appendix for gage by gage force comparisons for three of the four runs performed during the field test. Setup one data, in which the truck placement was at the centerline of the bridge, was excluded from this project due to corrupted data.

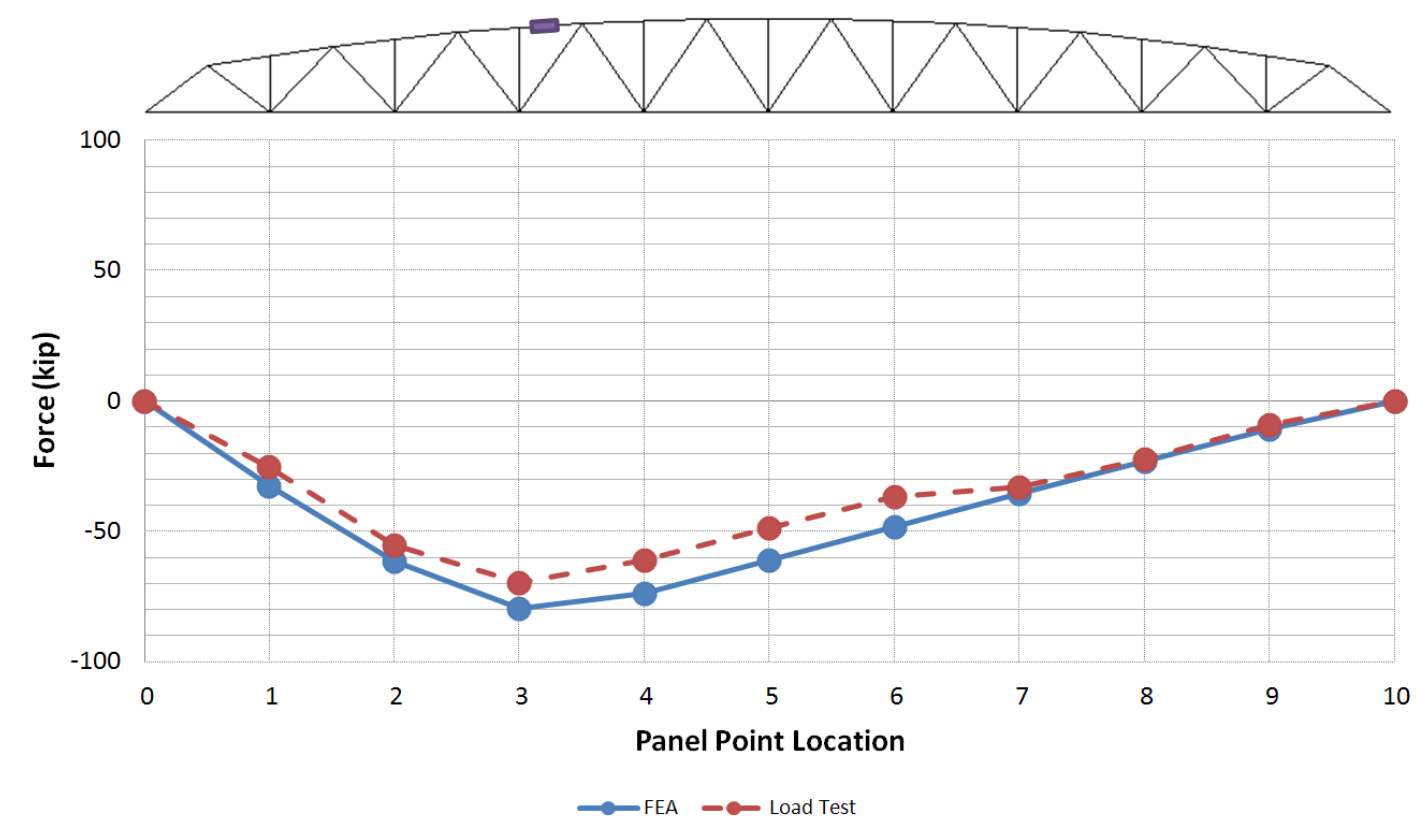

Figure 4.17: Top Chord Average Force Comparison 


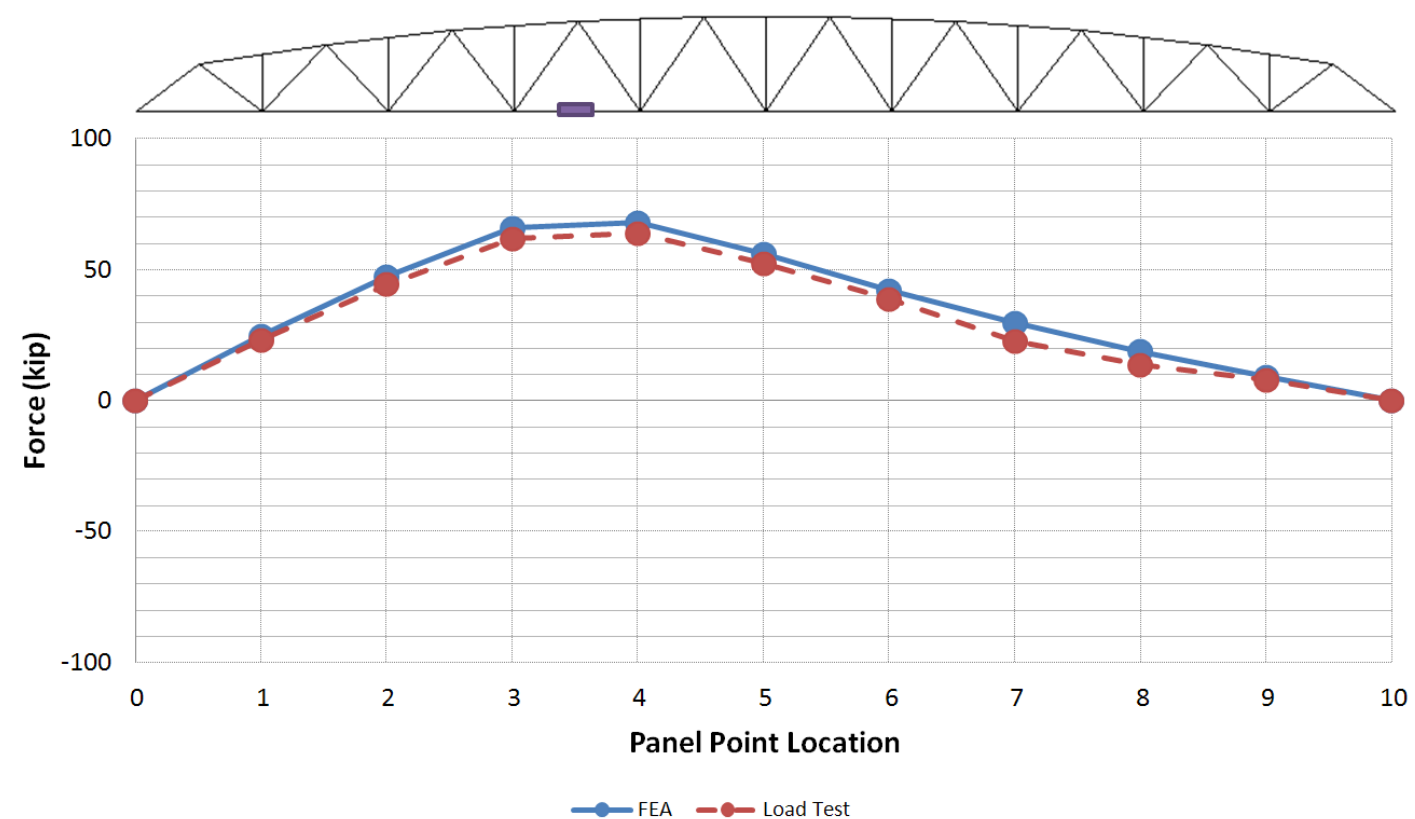

Figure 4.18: Bottom Chord Average Force Comparison

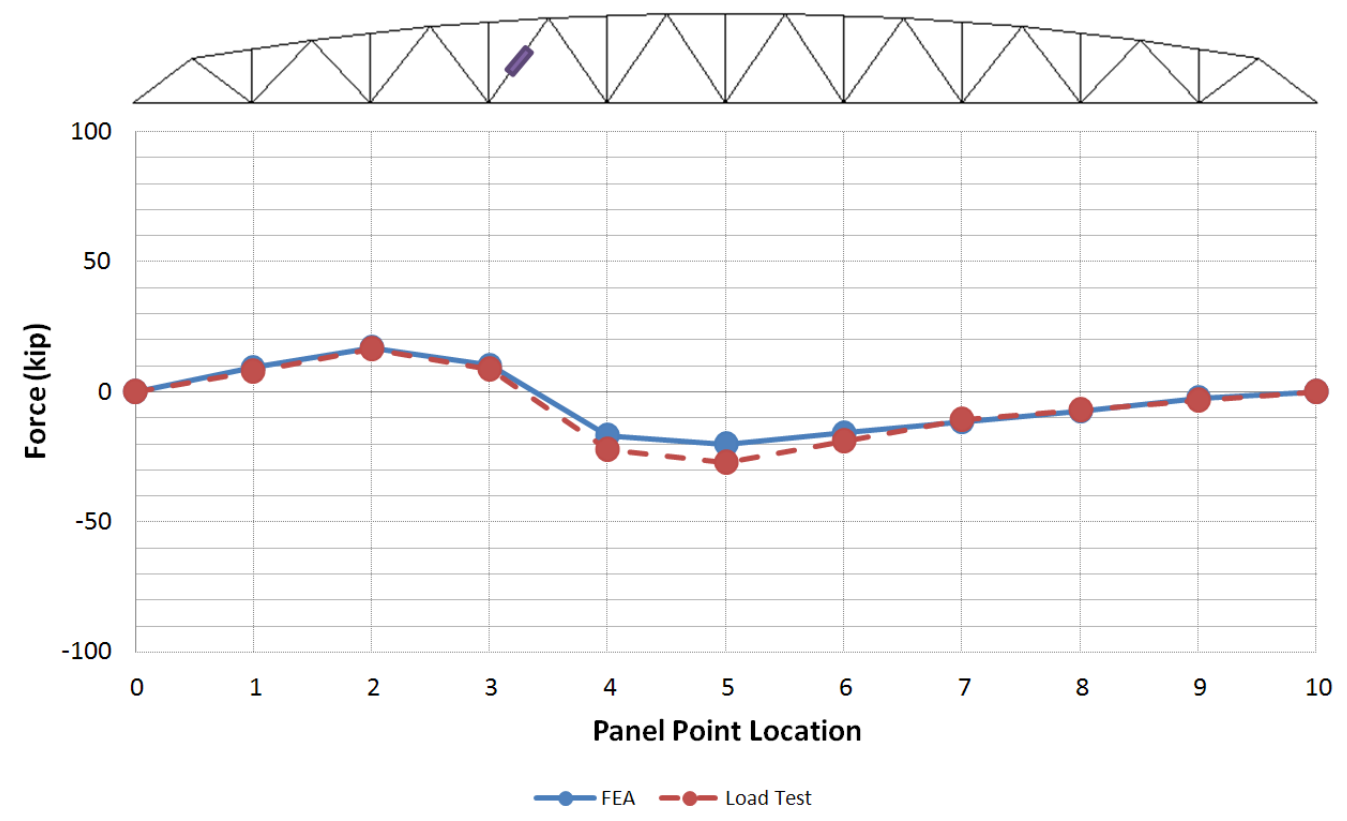

Figure 4.19: Diagonal Chord Average Force Comparison 


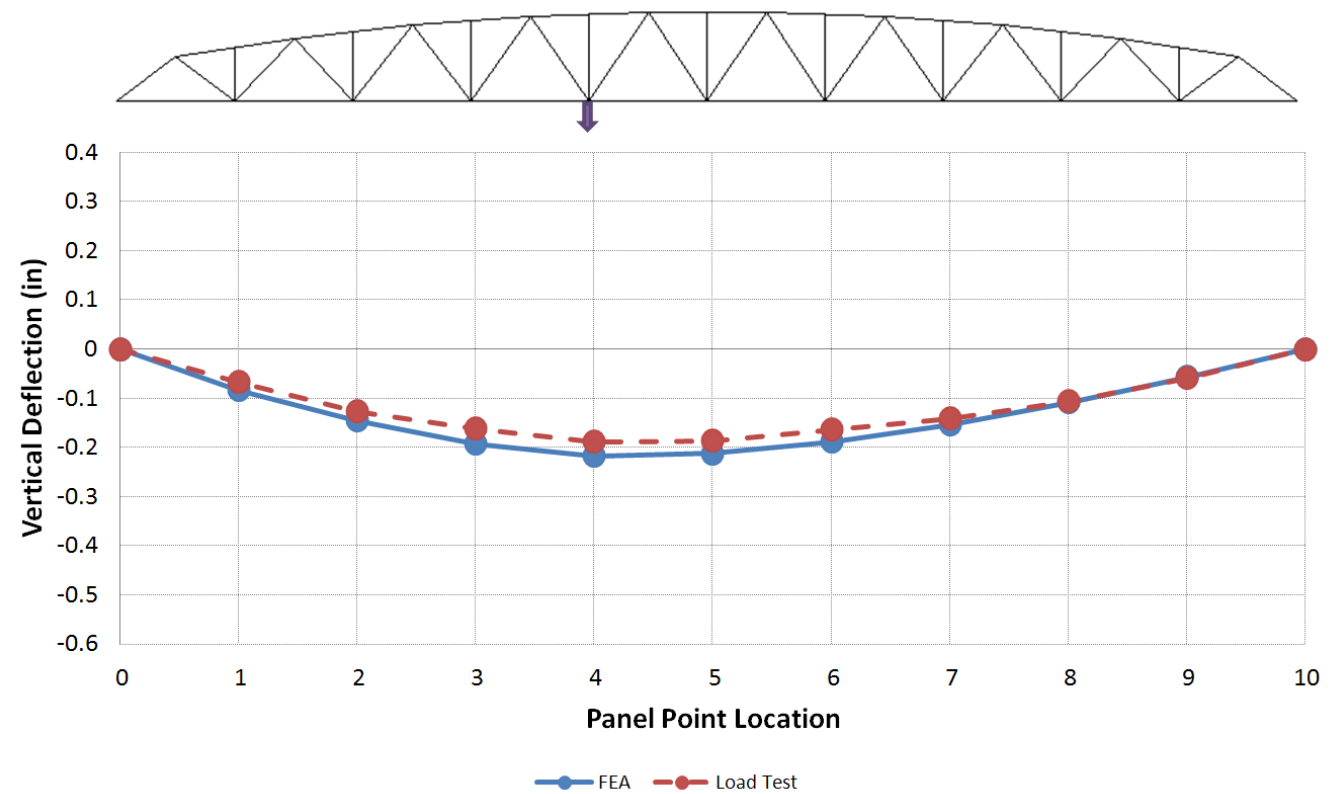

Figure 4.20: Vertical Deflection at Panel Point 4

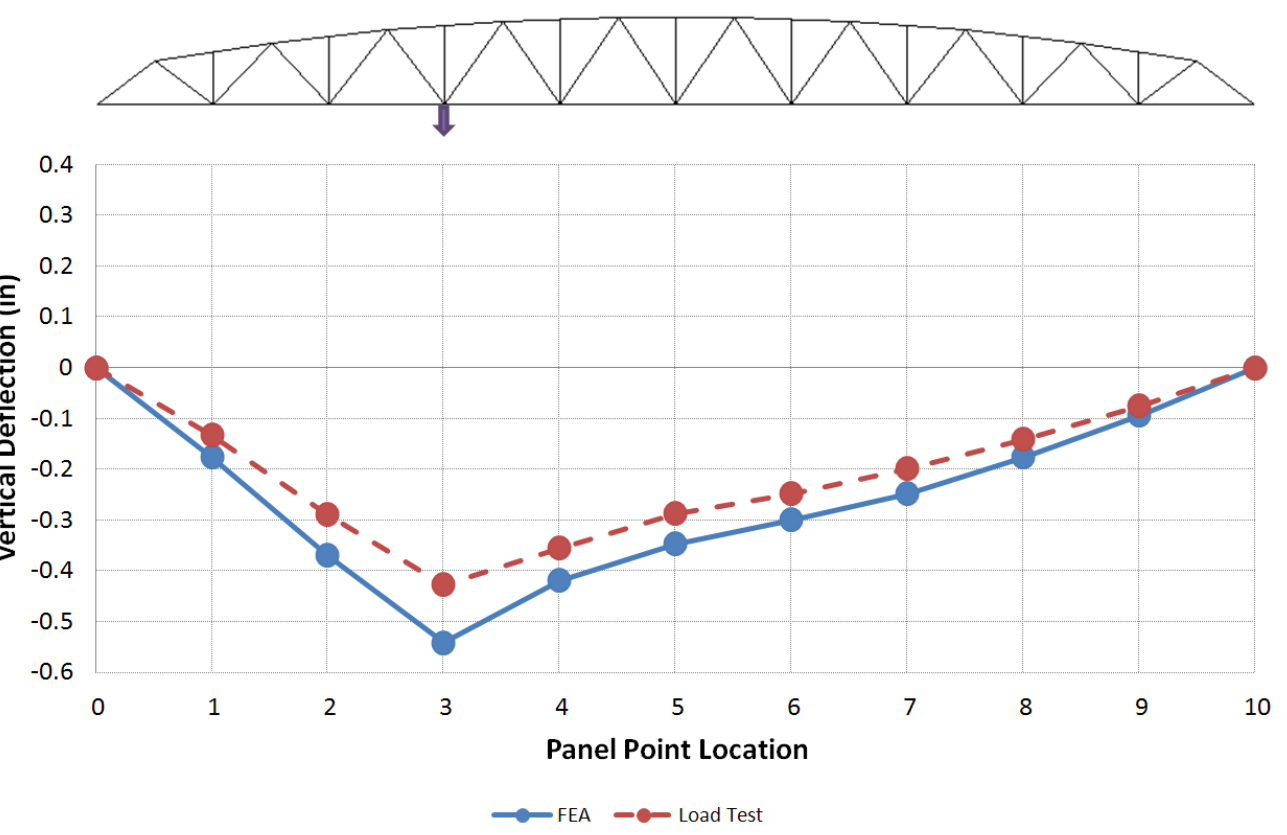

Figure 4.21: Vertical Deflection of Floor Beam 3 


\subsection{CONCLUSIONS}

As shown in the graphical comparisons in Section 4.6, the finite element modeling technique was shown to correlate well with the field test data. This enabled the continued use of the finite element model to complete redundancy analyses on the Little Mill Creek bridge. These redundancy analyses are discussed in the following chapter. 


\section{Chapter 5: Redundancy Assessment of Little Mill CreeK BRIDGE}

\subsection{INTRODUCTION}

As presented in Sections 4.6 and 4.7, the finite element model was found to accurately represent the behavior of the Little Mill Creek bridge. This enabled redundancy analyses to be performed on the bridge; presented in this chapter are descriptions of the cases assessed along with the results from each assessment.

\subsection{SELECTED MEMBERS FOR REDUNDANCY EVALUATION}

The focus of this redundancy evaluation was determining the system's damaged capacity due to the removal of bottom chord elements. These were chosen for two main reasons:

- These members are elements in axial tension which are designated as fracture-critical members.

- These members are built-up sections, allowing for internal member redundancy investigations as well as damaged capacity evaluations.

\subsubsection{Bottom Chords}

The Little Mill Creek bridge is symmetrical about both its longitudinal and transverse axes, which allowed for a significant decrease in the number of cases assessed. Therefore, only half of the west truss line was chosen for evaluation which included five bottom chord cases. The procedure described in Section 3.4 is employed to evaluate each of the bottom chord cases for redundancy. To simulate the loss of a specific member, the finite element mesh was edited by deleting the respective nodes and elements associated with both channels in the built-up section bottom chord being evaluated. Truck placement was determined as described in Section 3.2 and the longitudinal axle placements for each bottom chord case are given in Figure 5.1. 

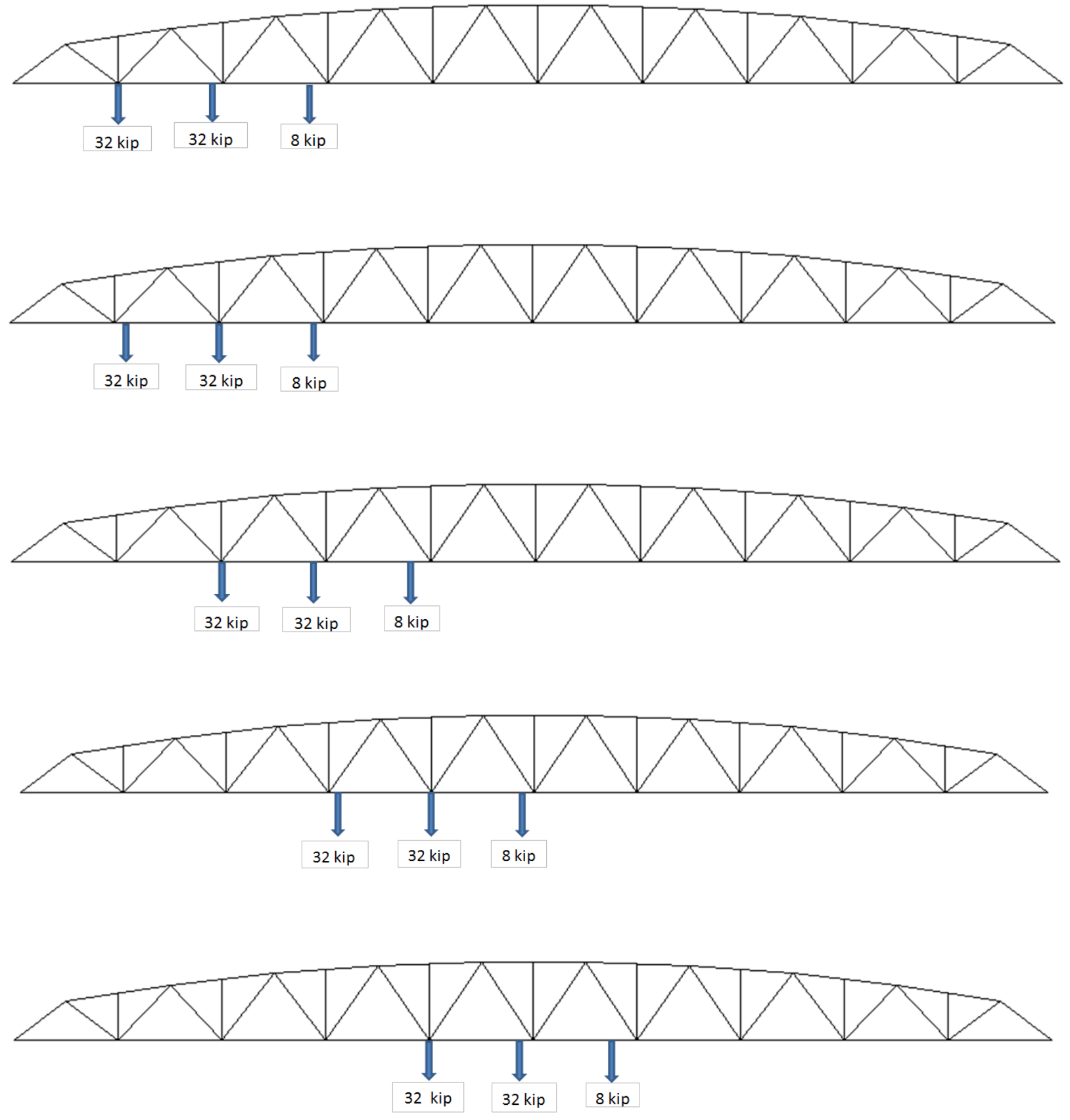

Figure 5.1: Bottom Chords 1-5 Longitudinal Truck Placements 


\subsubsection{Bottom Chord 4}

As stated in Section 5.2.1, the nodes and elements associated with both the interior and exterior channel which form a built-up bottom chord were deleted to simulate the loss of a member. In order to further assess these bottom chords as a built-up section, bottom chord 4 was chosen to perform more extensive evaluations on. This member was specifically chosen as it was one of those instrumented in the Little Mill Creek bridge field test discussed in Chapter 4. For this evaluation, an additional case was assessed by deleting only those nodes and elements associated with the exterior channel in bottom chord 4. This assessment allowed a check of internal member redundancy to be performed. The same truck placement shown in Figure 5.1 was employed for this individual component check as was employed for the full removal of bottom chord 4. Again, the procedure described in Section 3.4 was incorporated to evaluate this case.

\subsection{DisCUSSION OF REDUNDANCY ANALYSIS RESUltS}

Contained in this section is a presentation of the redundancy analyses performed along with a discussion of results from each.

\subsubsection{Bottom Chord Redundancy Evaluations}

Table 5.1 displays the redundancy analysis results from the bottom chord cases. As shown, the redundancy factors are all below the previously defined $R_{d}$ limit of 0.5 meaning each of these cases would be described as nonredundant according to current limits. However, it should be noted these limits were calibrated based upon beam bridges and should be recalibrated to be pertinent to truss bridges as well (see Section 2.5.1). 


\begin{tabular}{ccccccc} 
Bottom Chord & $\boldsymbol{\sigma}_{\text {DL }}$ & $\boldsymbol{\sigma}_{\text {DL+LL }}$ & $\boldsymbol{\sigma}_{\text {LL }}$ & $\mathbf{L F}_{\mathbf{1}}$ & $\mathbf{L F}_{\mathbf{d}}$ & $\mathbf{R}_{\mathbf{d}}$ \\
\hline 1 & 1.571 & 3.479 & 1.908 & 25.377 & 1.675 & 0.066 \\
2 & 3.252 & 6.991 & 3.739 & 12.504 & 2.333 & 0.187 \\
3 & 3.046 & 6.803 & 3.757 & 12.498 & 2.138 & 0.171 \\
4 & 3.923 & 8.778 & 4.855 & 9.491 & 2.447 & 0.258 \\
5 & 3.677 & 8.191 & 4.513 & 10.263 & 2.502 & 0.244
\end{tabular}

Table 5.1: Bottom Chord Redundancy Analysis Results

\subsubsection{Bottom Chord 4: Internal Member Redundancy Evaluation}

Table 5.2 displays the results of the two cases which were analyzed for bottom chord 4 (BC 4). The first case was evaluated by deleting only the nodes and elements associated with the exterior chord; this case did meet the redundancy limit for $\mathrm{R}_{\mathrm{d}}$ as the value is greater than 0.5 . This analysis led to the determination that $\mathrm{BC}-4$ did exhibit internal member redundancy by the definition given in Section 2.5.1. In addition, as shown in Table 5.2, as the truss was only subjected to an exterior channel cut, the bridge was able to withstand larger amounts of live loads, which therefore yielded a much higher $\mathrm{R}_{\mathrm{d}}$ value.

\begin{tabular}{ccccccc} 
BC 4 Case & $\boldsymbol{\sigma}_{\text {DL }}$ & $\boldsymbol{\sigma}_{\text {DL+LL }}$ & $\boldsymbol{\sigma}_{\mathrm{LL}}$ & $\mathbf{L F}_{\mathbf{1}}$ & $\mathbf{L F}_{\mathbf{d}}$ & $\mathbf{R}_{\mathbf{d}}$ \\
\hline \hline Exterior Chord & 3.963 & 8.731 & 4.768 & 9.656 & 5.074 & 0.525 \\
Full Chord & 3.923 & 8.778 & 4.855 & 9.491 & 2.447 & 0.258
\end{tabular}

Table 5.2: Bottom Chord 4 Redundancy Analysis Results

\subsection{CONCLUSIONS}

It has been shown that, according to the limits set forth in NCHRP Report 406, the full cut bottom chord cases chosen for analysis in this chapter did not meet redundancy requirements. However, it should be noted that these limits were not calibrated for trusses. In addition, it was observed that the truss' capacity under damaged conditions was quite impressive; in some cases, the truss was able to withstand as much as ten design trucks with an entire member removed. 
Therefore, it is apparent that future calibration of the limits associated with damaged condition capacity of truss systems is in order to better assess redundancy in these types of bridges. 


\section{Chapter 6: Summary AND CONCluding REMARKS}

\subsection{Project Summary}

The focus of this project was to develop redundancy protocols for steel truss bridges. As stated in Section 1.2, the objectives and scope of this project were as follows:

- A literature review focused on determining factors affecting redundancy in bridges was conducted.

- A set of redundancy protocols along with limit states to evaluate redundancy were developed. These were based upon the limit states and protocols set forth in NCHRP Report 406 (Ghosn and Moses, 2007).

- A physical load test was then performed on an existing steel truss bridge to ensure the validity of the finite element modeling technique utilized in this project.

- Finally, a redundancy assessment was performed on the bridge on which the load test was performed.

\subsection{RECOMMENDATIONS FOR FUTURE WORK}

The author recommends the following tasks for future work and/or expansions to this project.

- Perform cut-chord iterations on the remaining tension members of the representative steel truss bridge in this thesis along with those in other representative bridges.

- Investigate more appropriate protocols for the functionality limit state.

- While this study relied on the theory of influence lines to place load trucks on the finite element model, it may be necessary to further examine these placements. Once a member is "cut" from the model, the load truck placement based on the influence lines for the intact structure may not produce the maximum load effects for the damaged structure.

- Develop and/or calibrate new redundancy limits which can be employed to evaluate levels of redundancy present in any truss bridge configuration. 


\section{REFERENCES}

Agrawal, A. K., A. Kawaguchi and Z. Chen. "Deterioration Rates of Typical Bridge Elements in New York." Journal of Bridge Engineering 15.4 (2010): 419-429.

American Association of State Highway and Transportation Officials. AASHTO LRFD Bridge Design Specifications. Washington, DC: AASHTO, 2010.

Ayyub, Bilal M. and Ahmed Ibrahim. "Posttensioned Trusses: Reliability and Redundancy." Journal of Strucural Engineering 116.6 (1990): 1507-1521.

Bondi, Robert W. "Adding Redundancy to 2-Eyebar Members in a Cantilever Truss Bridge." 2nd International Bridge Conference. Pittsburgh, PA, 1985. 47-53.

Bridge Diagnostics, Inc. STS-WiFi Operations Manual. Boulder, n.d.

Bridge Engineering Software \& Technology (BEST) Center. "Report on the Determination of Redundancy of the U.S. Bridge Corporation Bridge 3000." University of Maryland, 2000.

Commonwealth of Pennsylvania Department of Transportation. "Penn DOT Design Manual Part 4." 2007.

Connor, Robert J., Robert Dexter and Hussam Mahmoud. "NCHRP Synthesis 354: Inspection and Management of Bridges with Fracture-Critical Details." 2005.

Daniels, J.H., W. Kim and J.L. Wilson. "Guidelines for Redundancy Design and Rating of TwoGirder Steel Bridges." NCHRP Report 319. 1988.

Frangopol, D. and R. Nakib. "Effects of Damage and Redundancy on the Safety of Existing Bridges." Third Bridge Engineering Conference. Denver, Colorado: TRB 1290, 1991.

Frangopol, Dan M. and James P. Curley. "Effects of Damage and Redundancy on Structural Reliability." Journal of Structural Engineering 113.7 (1987): 1533-1549.

Fu, Gongkang and Dan M. Frangopol. "System Reliability and Redundancy in a Multiobjective Optimization Framework." International Workshop on Structural System Reliability. Boulder, CO, 1988. 147-157.

Galambos, T.V., T.R. Leon and C.W. French. Inelastic Rating Procedures for Steel Beam and Girder Bridges. NCHRP Project 12-28. Washington, D.C.: TRB, 1992.

Galindez, N. and Karl E. Barth. "Evaluation of Flange Bending Stresses in Non-composite Steel I-girder Bridges During Construction." Bridge Structures: Assessment, Design and Construction 5.4 (2009): 189-203. 
Ghosn, M. and F. Moses. "Redundancy in Highway Bridge Superstructures, Report 406." 1997.

Ghosn, Michel and Fred Moses. "Evaluation of the Redundancy of Highway Bridges." 3rd International Workshop on Bridge Rehabilitation. Darmstadt, 1992.

Ghosn, Michel. "Evaluation of the Redundancy of Structural Systems." NSF Workshop on Structural System Reliability. Boulder, 1988.

Ghosn, Michel. "System Factors for Highway Bridge Superstructures." Journal of the Transportation Research Board (2005).

Ghosn, Michel, Fred Moses and Nikhil Khedekar. "Response Function and System Reliability of Bridges." Probabilistic Structural Mechanics: Advances in Structural Reliability Methods (1994): 220-236.

Heins, C.P. and H. Kato. "Load Redistribution of Cracked Girders." Journal of the Structural Division 108.ST8 (1982): 1909-1915.

Helba, Alaa and John B. Kennedy. "Parametric Study on Collapse Loads of Skew Composite Bridges." Journal of Structural Engineering 120.5 (1994).

Holth, Nathan and Rick McOmber. "Ambridge Bridge." 3 July 2006. HistoricBridges.org. 17 November 2011.

$<\mathrm{http}: / /$ www.historicbridges.org/bridges/browser/?bridgebrowser=pennsylvania/ambridg e/>.

Hovell, Catherine and Eric Williamson. "Evaluating Redudnancy in Trapezoidal Box-Girder Bridge Using Finite Element Analysis." Thesis. 2008.

Idriss, R.L., K.R. White and D.V. Jauregui. "After-Fracture Response of a Two-Girder Steel Bridge." Structures Congree '93. Irvine, CA, 1993. 1077-1082.

Kanno, Yoshihiro and Yakov Ben-Haim. "Redundancy and Robustness, or When is Redundancy Redundant?" Journal of Structural Engineering 137.9 (2011): 935-945.

Liao, Minmao, et al. "Nonlinear Finite-Element Analysis of Critical Gusset Plates in the I-35W Bridge in Minnesota." Journal of Structural Engineering 137.1 (2011): 59-68.

Michaelson, Gregory K. "Live Load Distribution Factors for Exterior Girders in Steel I-Girder Bridges." Thesis. West Virginia University, 2010.

National Cooperative Highway Research Program. "Redundancy in Highway Bridge Superstructures, Report 406." 1998.

National Steel Bridge Alliance. "Redundancy." NSBA. Steel Bridge Design Handbook. 2010. 
Nowak, Andrzej S. and Kevin R. Collins. Reliability of Structures. McGraw-Hill Higher Education, 2000.

Purvis, Ron L. "Inspection of Fracture Critical Bridge Members." Committee on Structures Maintenance, 1988.

Sandberg, Harold and Richard Parmelee. "Redundancy by Design - Its Implications." 3rd Annual International Bridge Conference. Pittsburgh, n.d.

Task Committee on Redundancy of Flexural Systems. "State-of-the-Art Report on Redundant Bridge Systems." Journal of Structural Engineering 111.12 (1985).

Yang, Lili. "Evaluation of Moment Redistribution for Hybrid HPS 70W Bridge Girders." Thesis. West Virginia University, 2004. 


\section{APPENDIX：Results OF Field TeST}

\section{A.1 Description Of Field Test DATA}

The following includes gage by gage comparisons between the Little Mill Creek bridge field test and the finite element model for setup 1 with the truck at the edge, setup 2 with the truck at the edge, and setup 2 with the truck at the centerline. As stated in Section 4.6, the data for setup 1 with the truck at the centerline has been excluded from this project as the data was corrupted during the field test. The naming convention for LVDTs begins with LVDT followed by the panel point location followed by the side of the bridge the instrument was placed. For example, the LVDT placed at panel point 3 on the east side of the bridge is named LVDT-P3East. The naming convention for the strain gages begins with $G$ followed by the member the instrument was on followed by the gage number. Figures A.1 through A.4 show the gage numbering and locations for specific members as instrumented. For example, the gage located on the top of the top chord web is named G-TC-3.

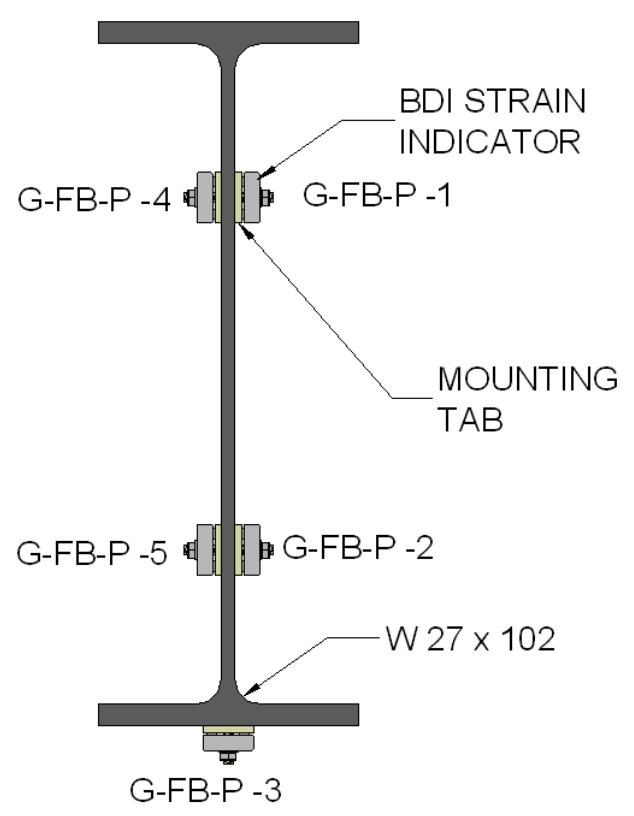

Figure A.1: Floor Beam Strain Gage Numbering 


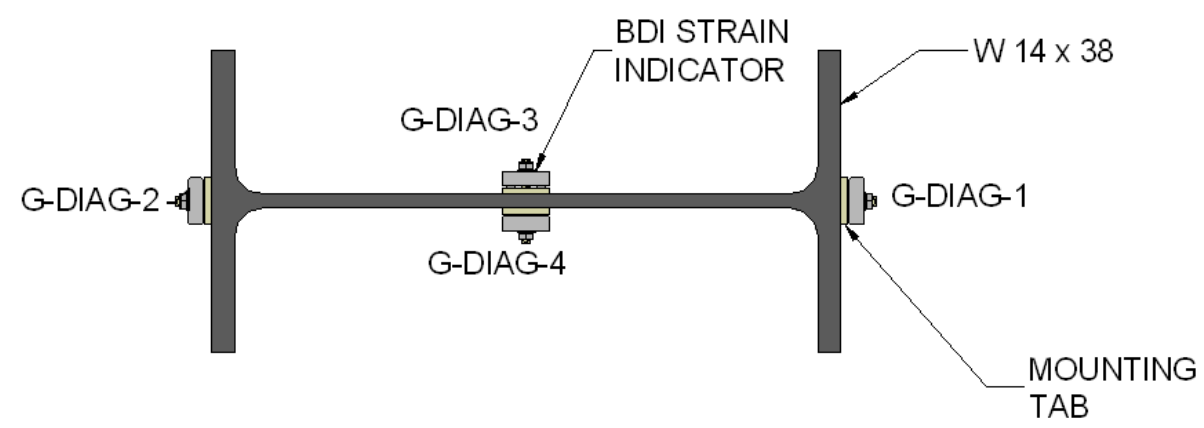

Figure A.2: Diagonal Strain Gage Numbering

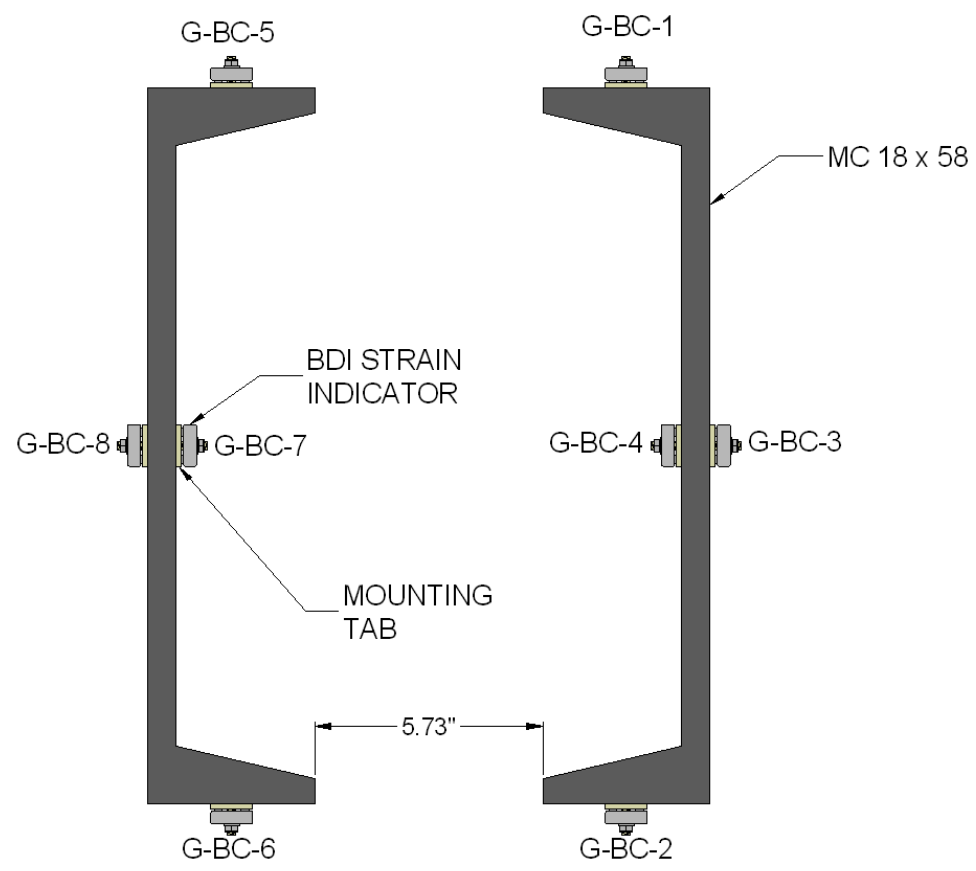

Figure A.3: Bottom Chord Strain Gage Numbering 


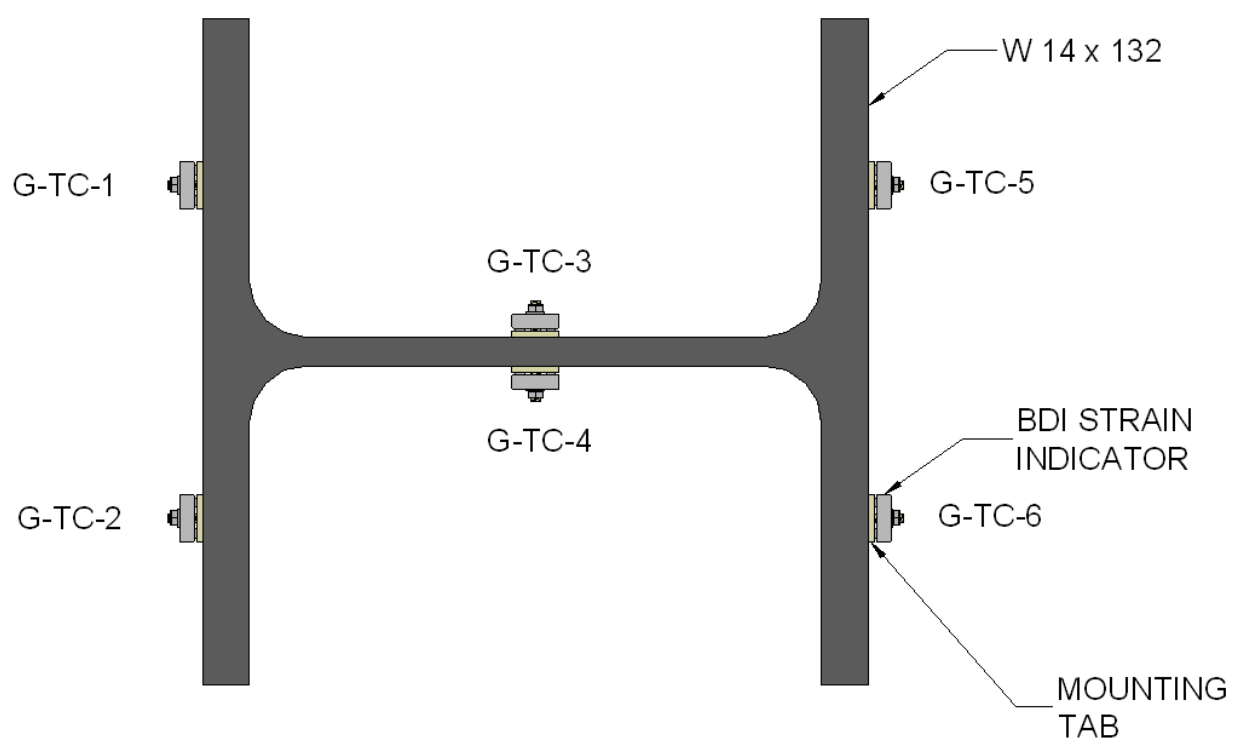

Figure A.4: Top Chord Strain Gage Numbering

\section{A.2 Run 1 (Setup \#1, Edge Truck Run)}

\section{LVDT-P3-East}

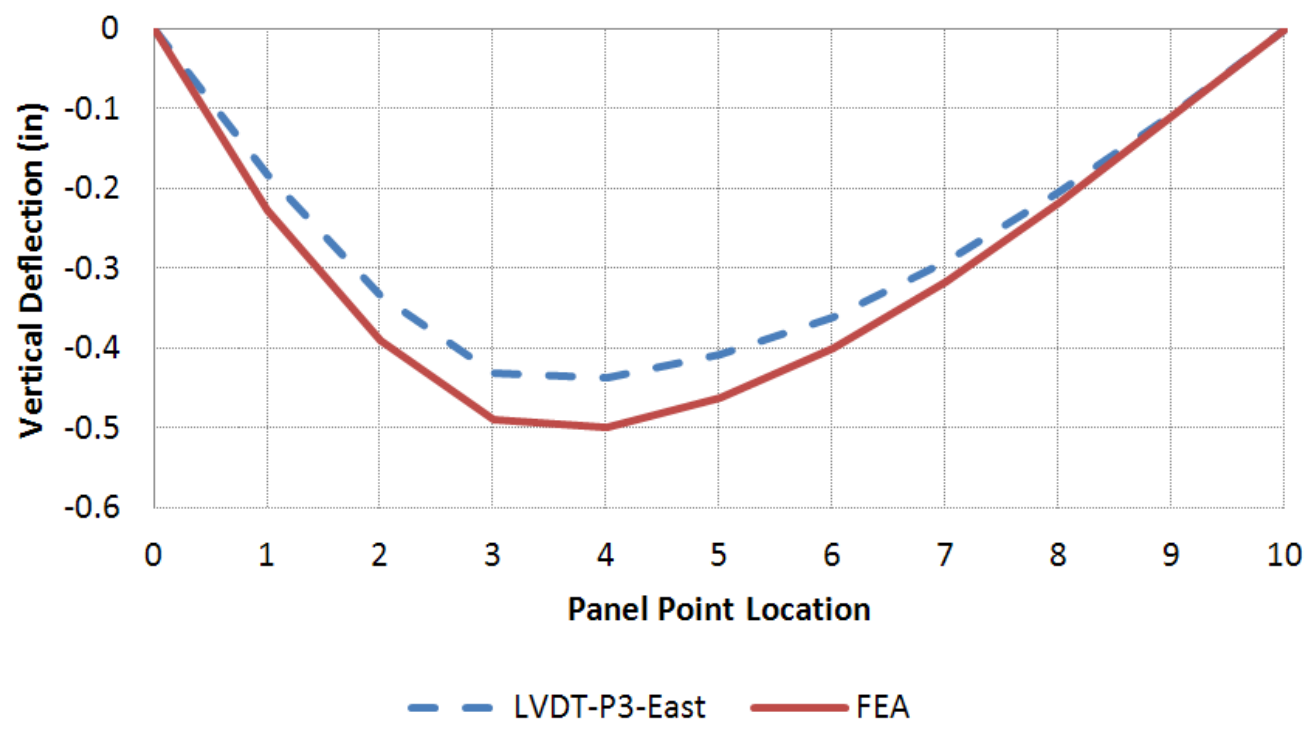




\section{LVDT-P3-FB}

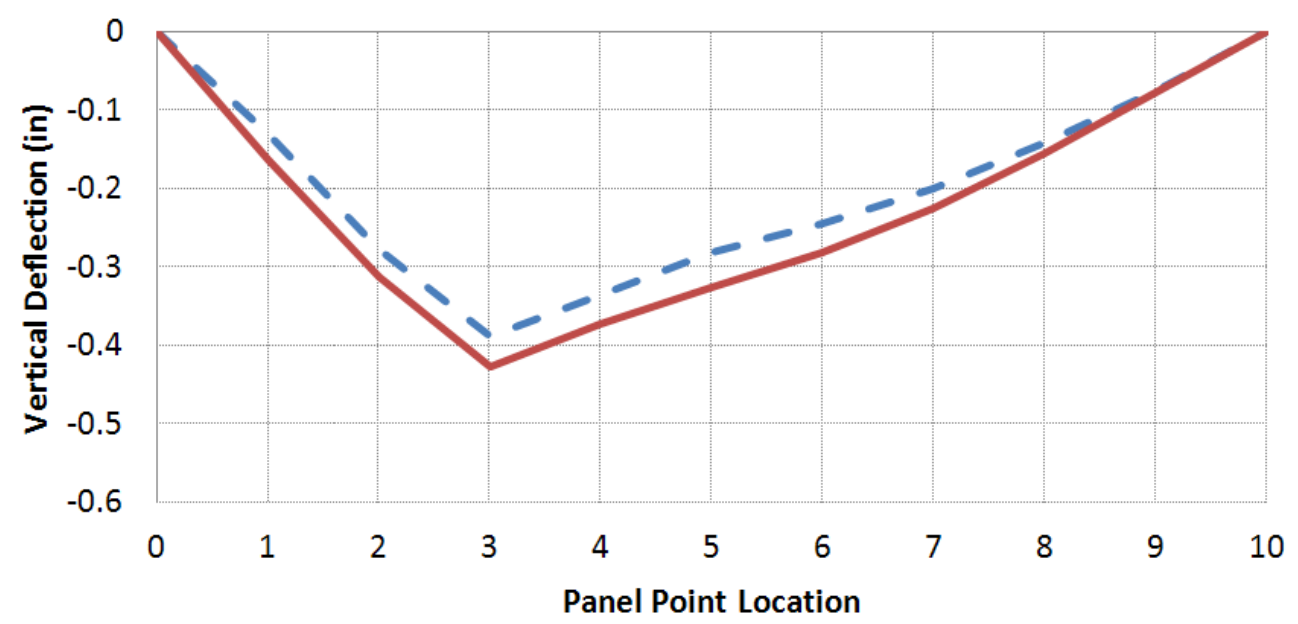

- LVDT-P3-FB TEA

\section{LVDT-P3-West}

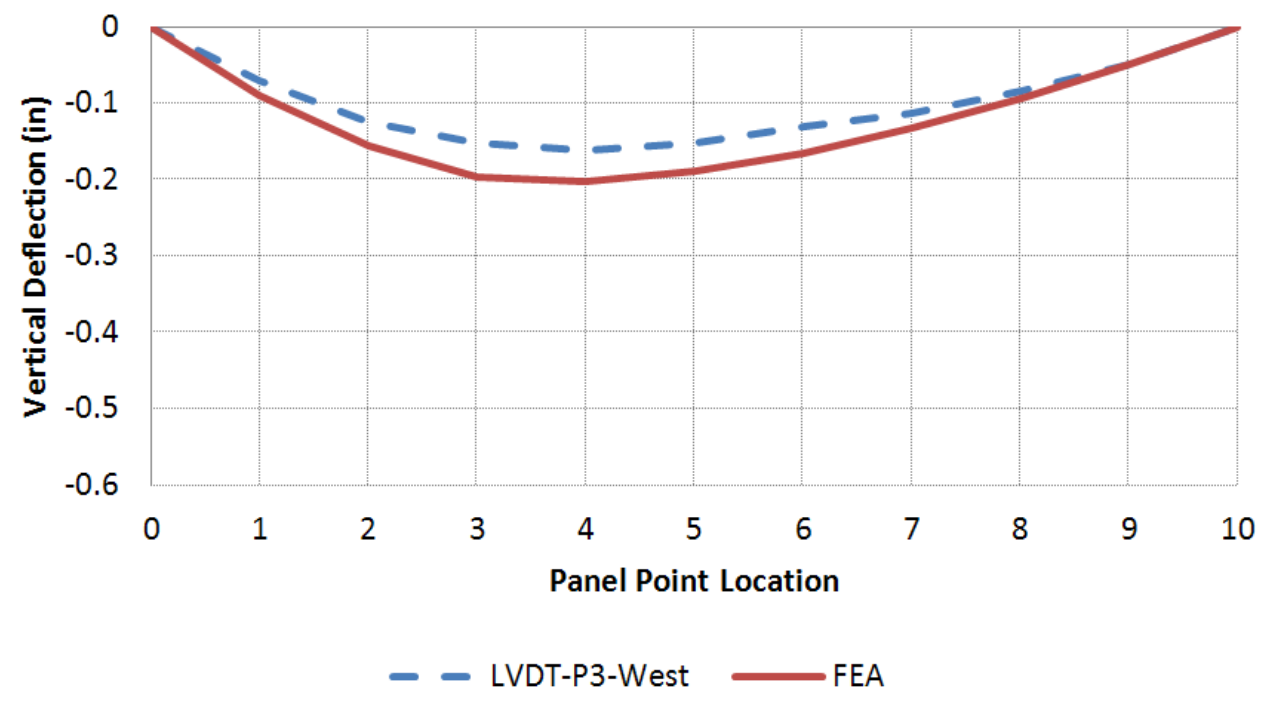




\section{LVDT-P4-East}

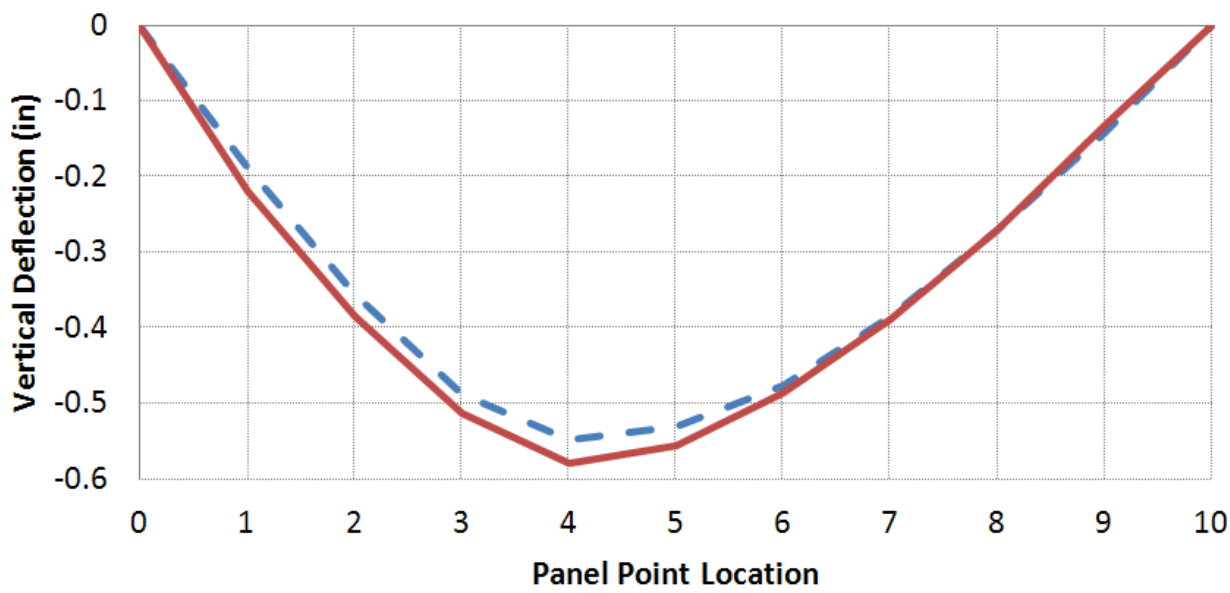

$$
\text { - LVDT-P4-East FEA }
$$

\section{LVDT-P4-FB}

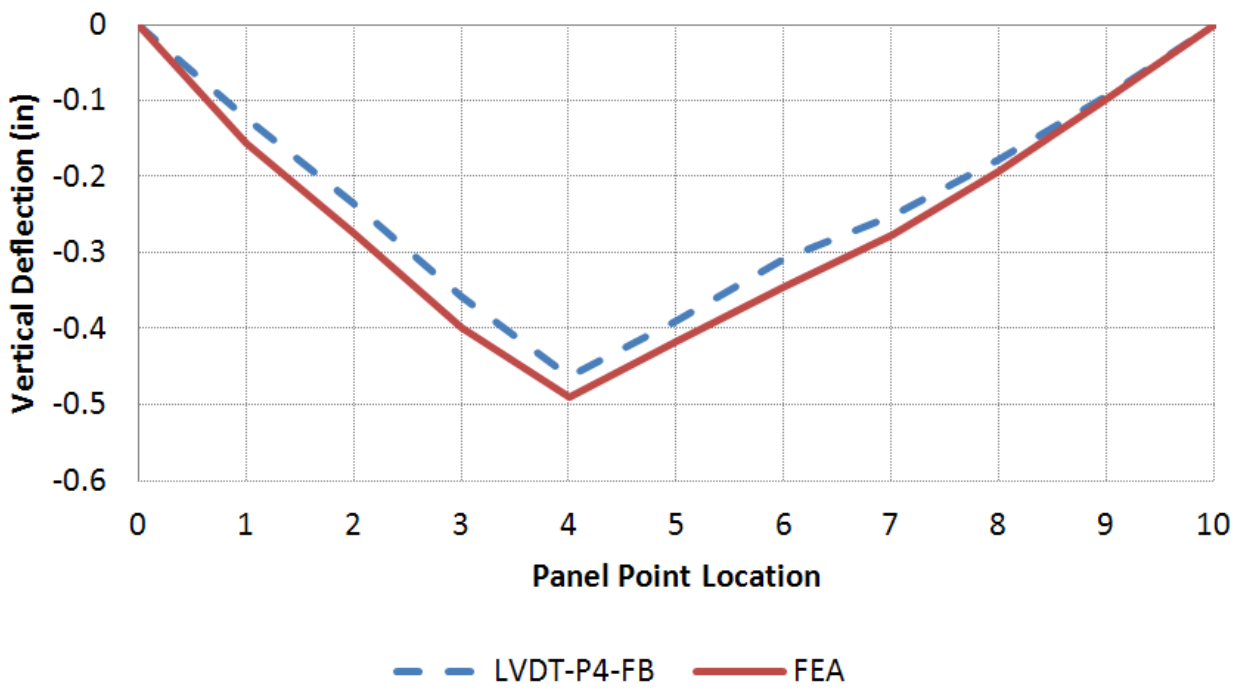




\section{LVDT-P4-West}

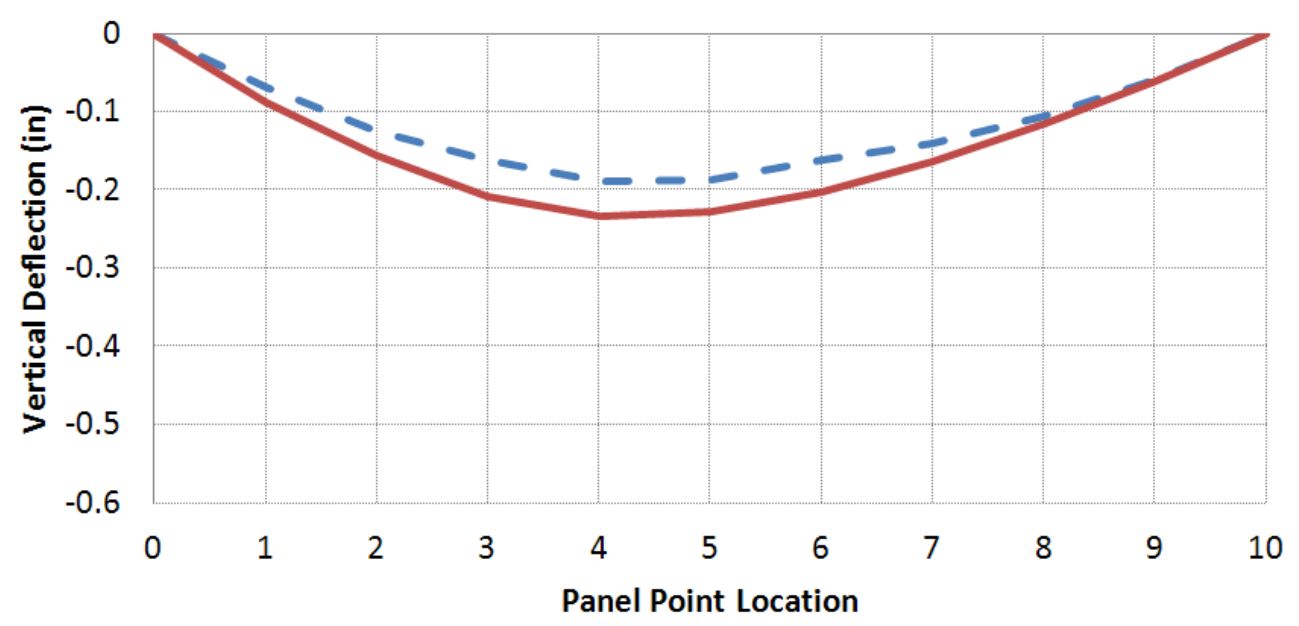

- - LVDT-P4-West $\longrightarrow$ FEA

\section{G-DIAG-2}

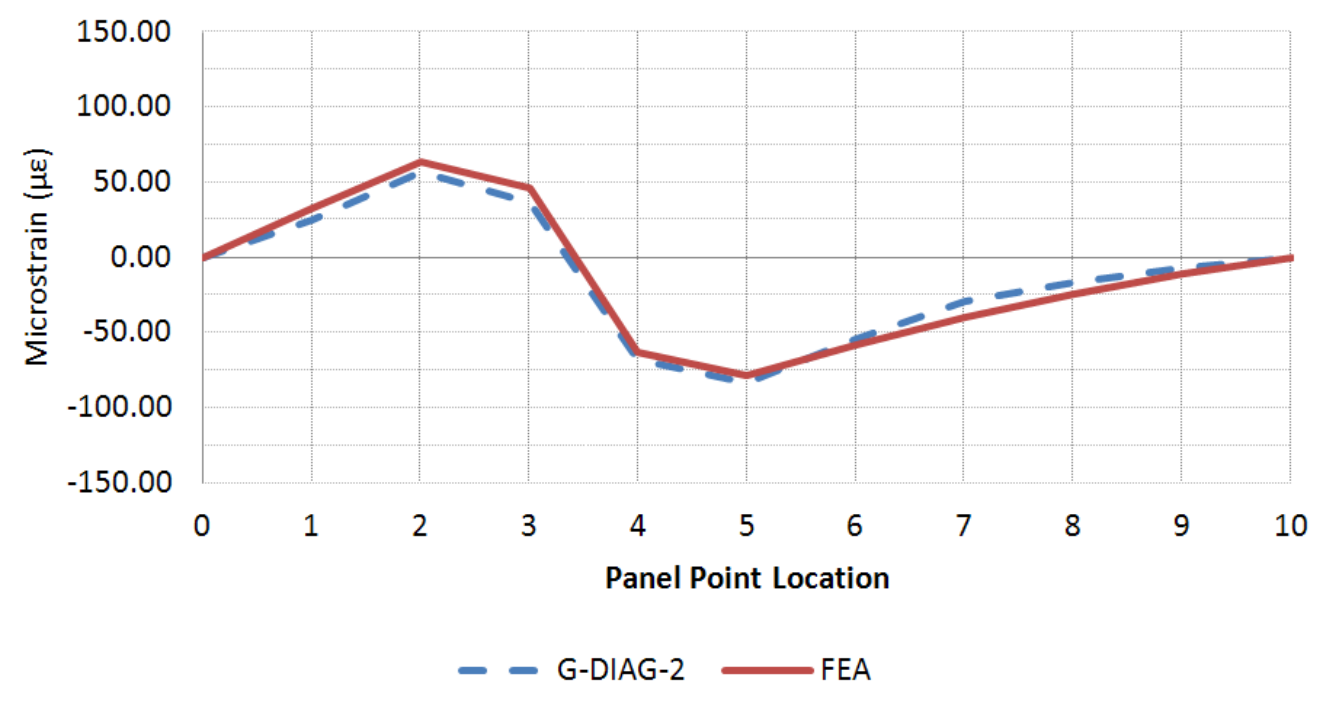




\section{G-DIAG-4}

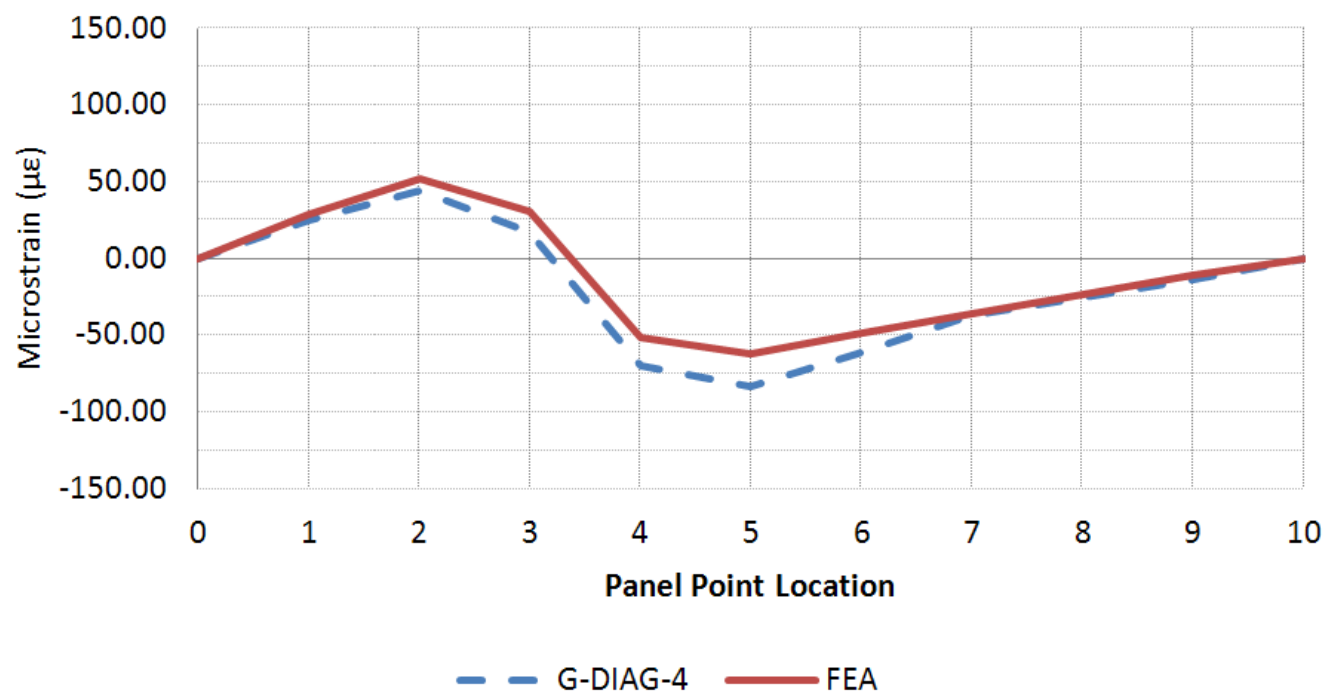

G-FB-P3-1

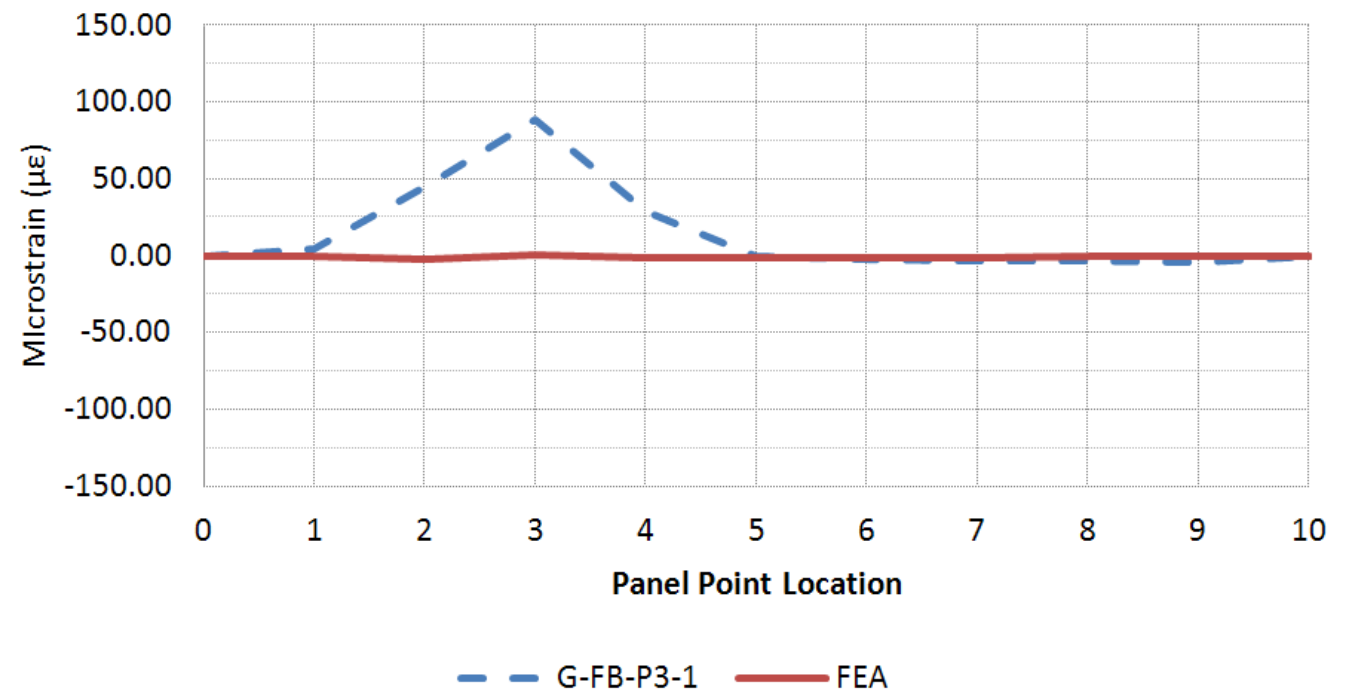


G-FB-P3-2

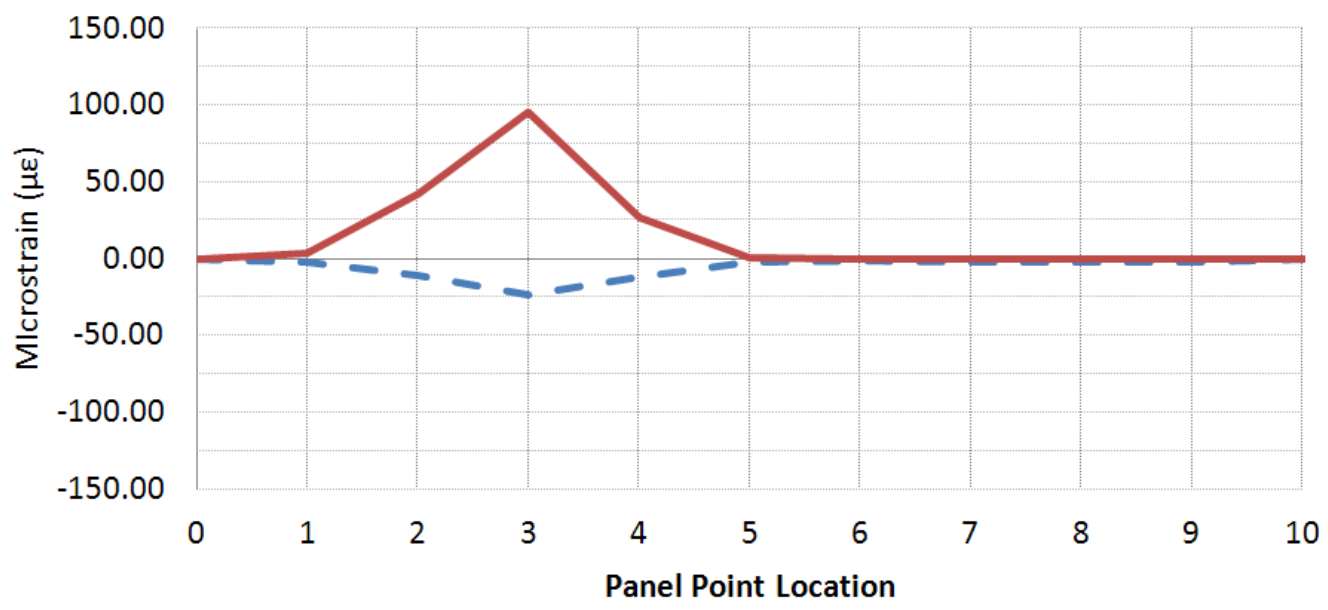

- G-FB-P3-2 FEA

\section{G-FB-P3-3}

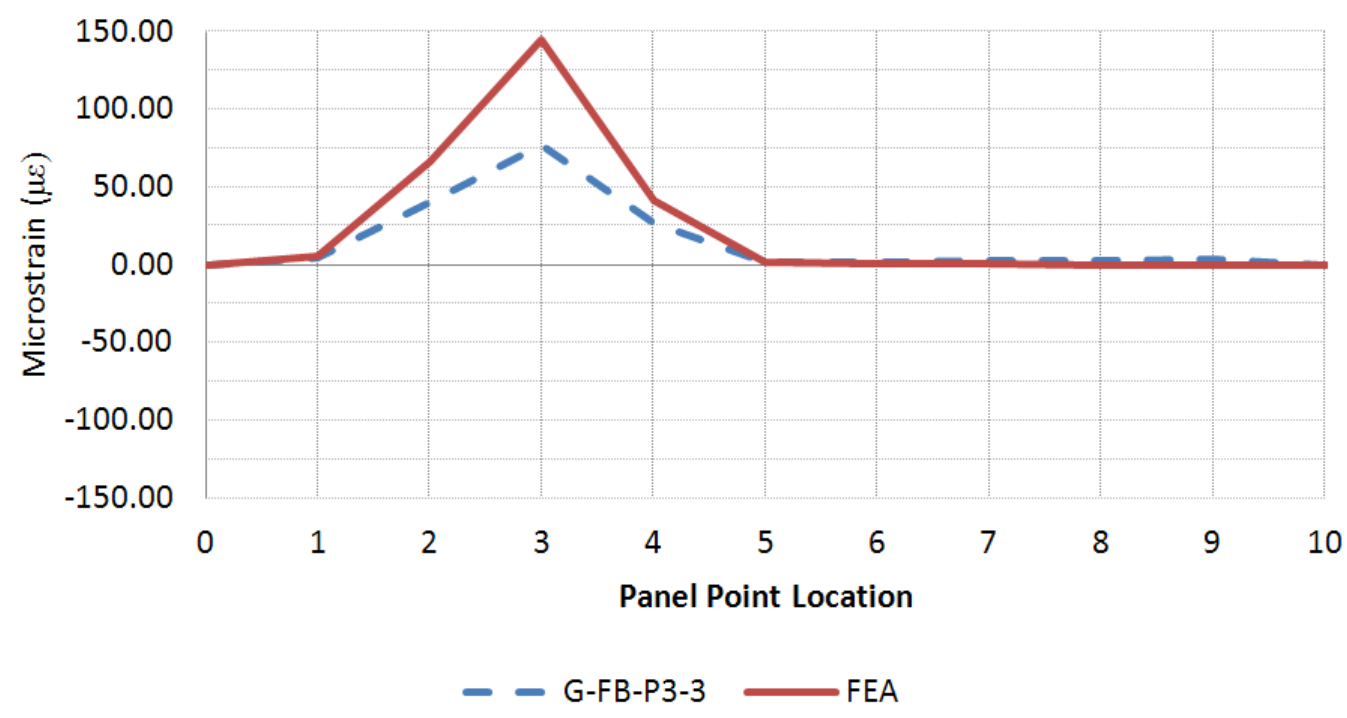




\section{G-FB-P3-4}

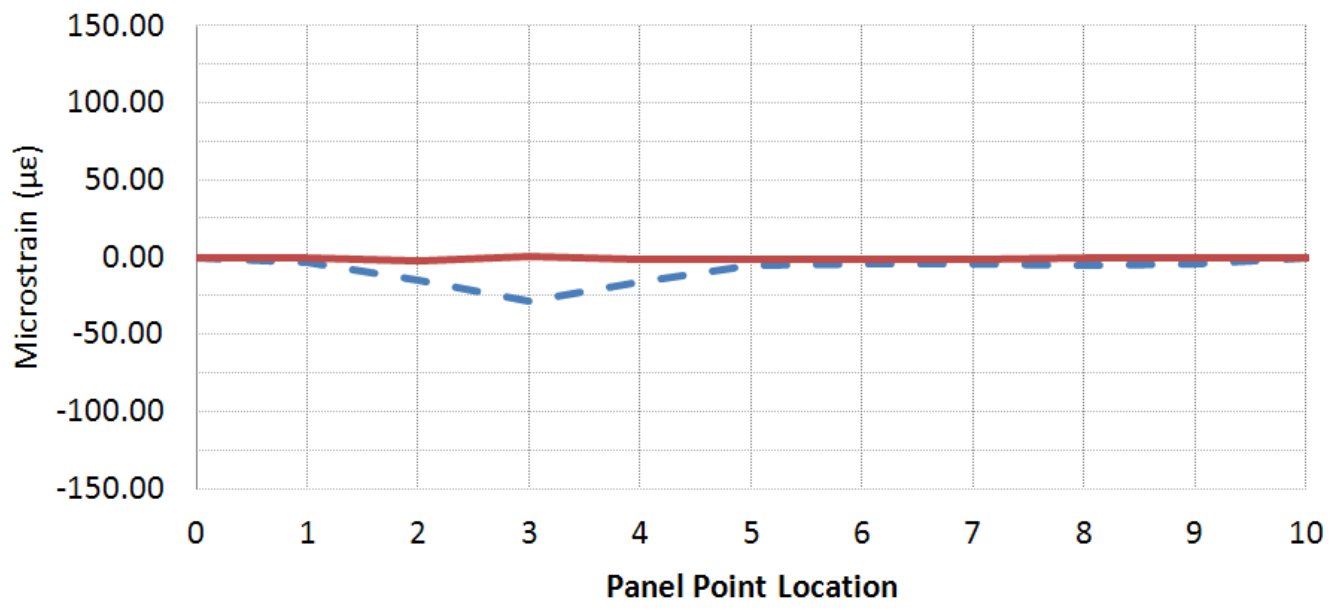

- G-FB-P3-4 FEA

\section{G-FB-P3-5}

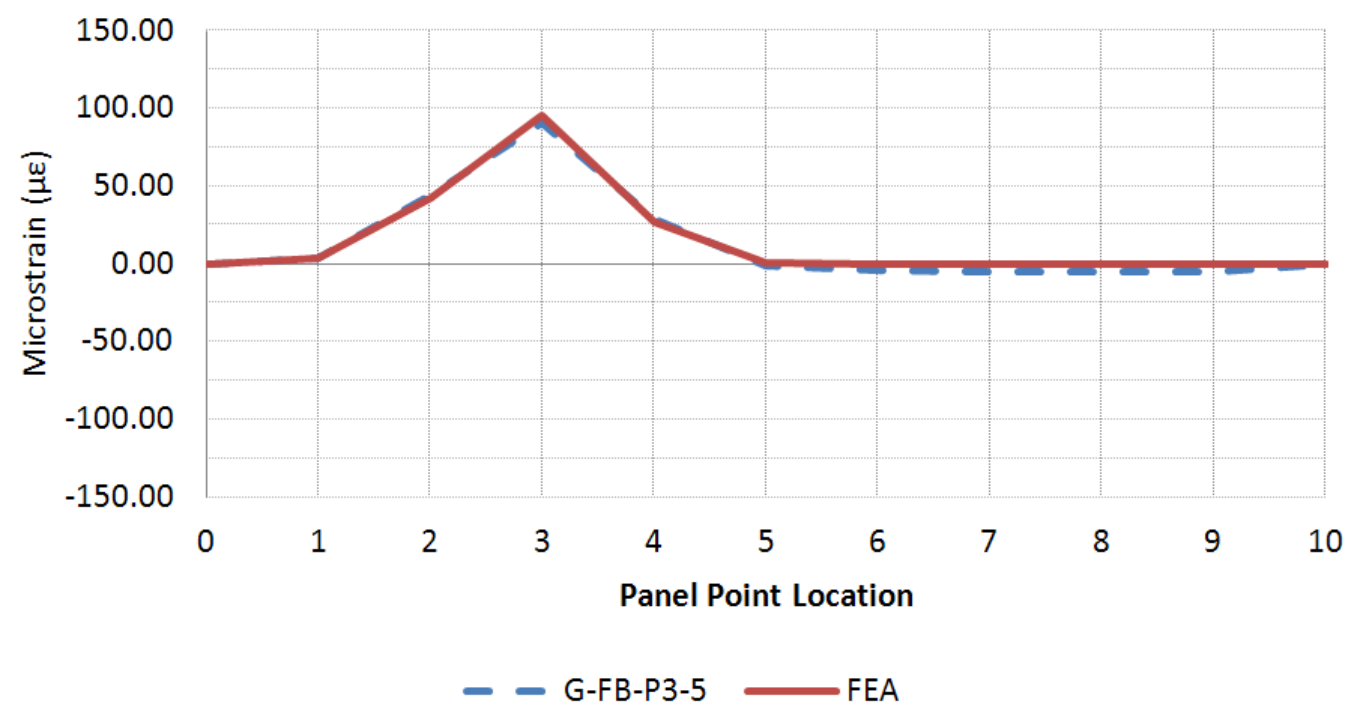


G-FB-P4-1

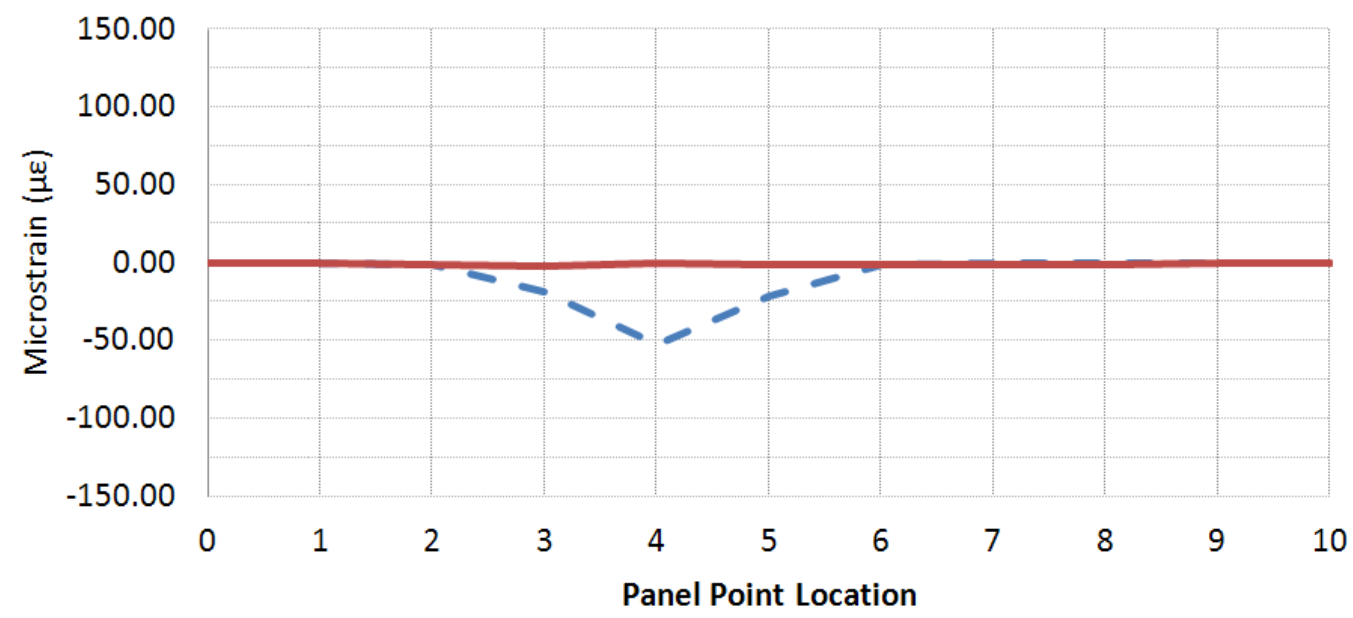

- G-FB-P4-1 FEA

\section{G-FB-P4-2}

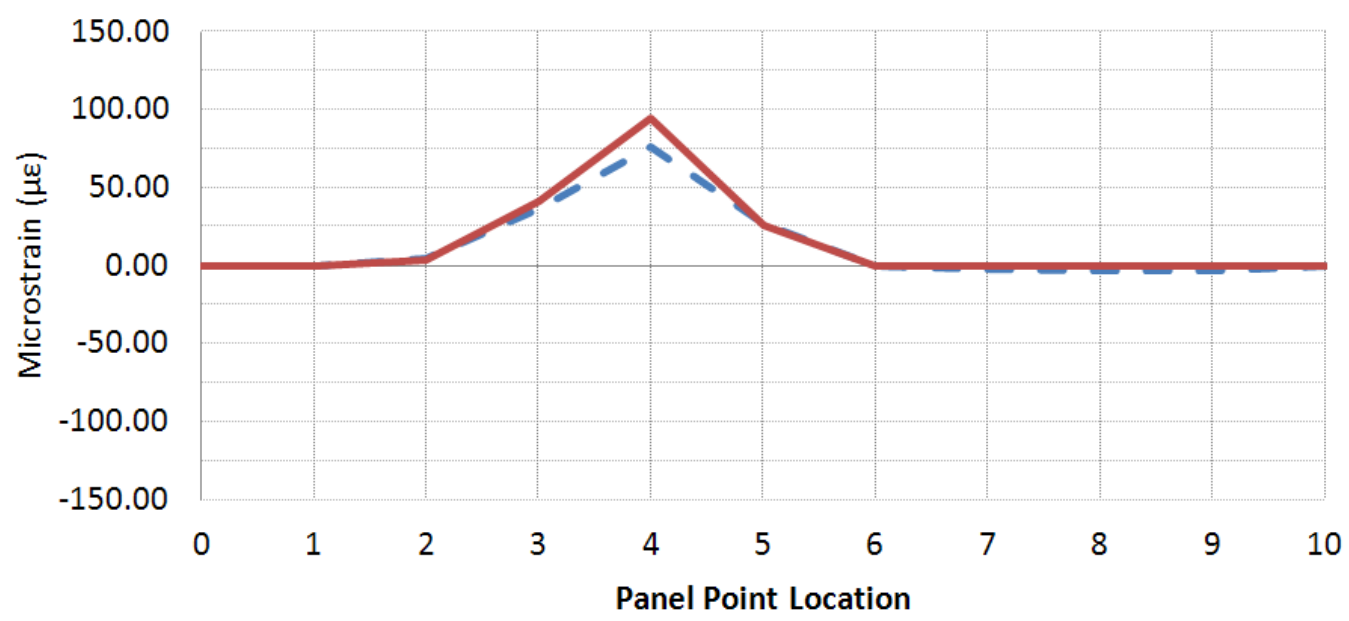

- G-FB-P4-2 FEA 


\section{G-FB-P4-3}

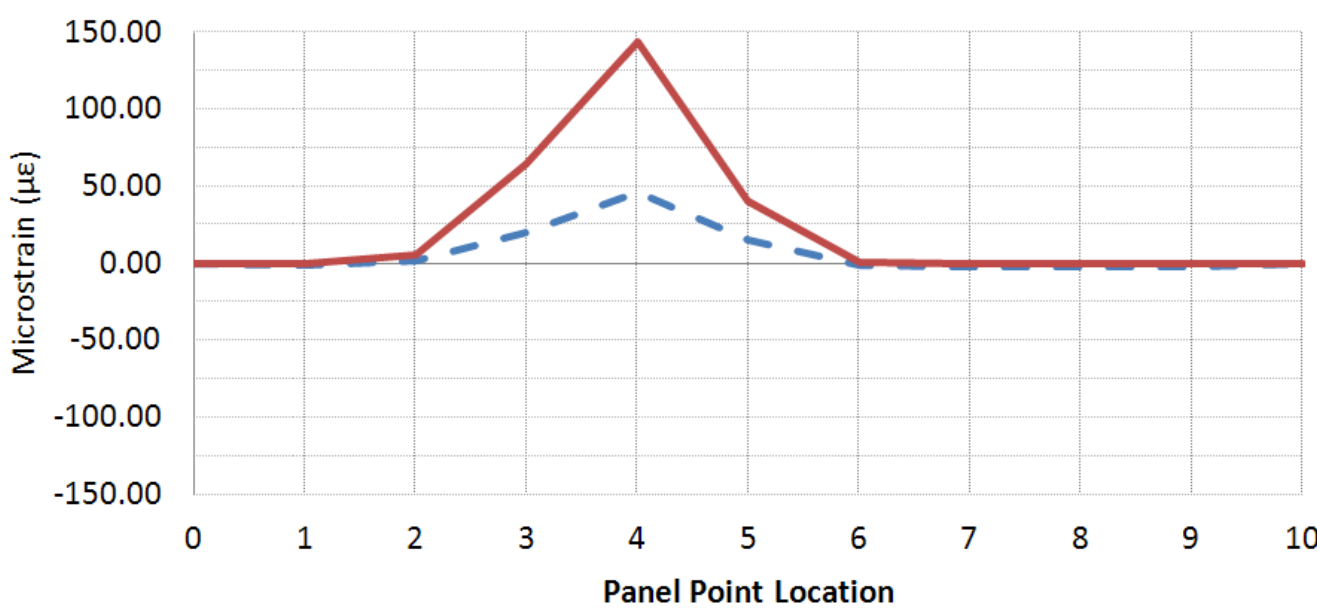

- G-FB-P4-3 FEA

\section{G-FB-P4-4}

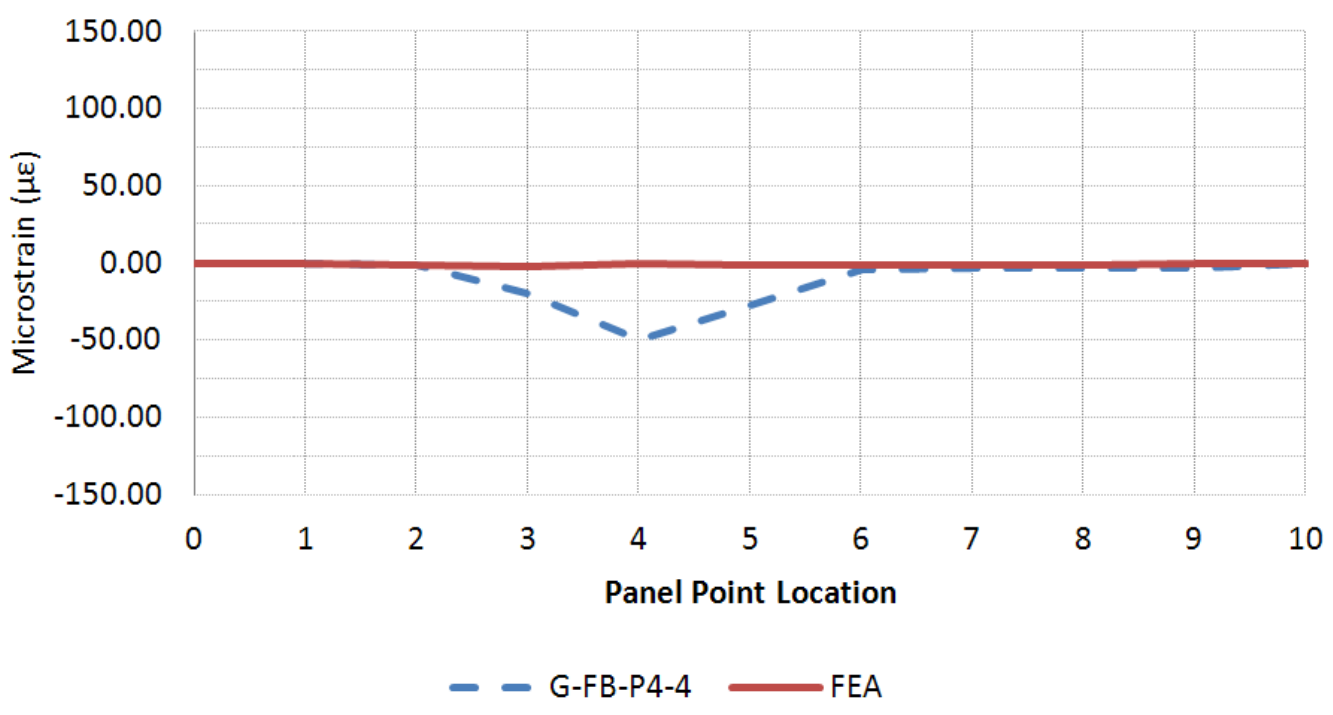


G-FB-P4-5

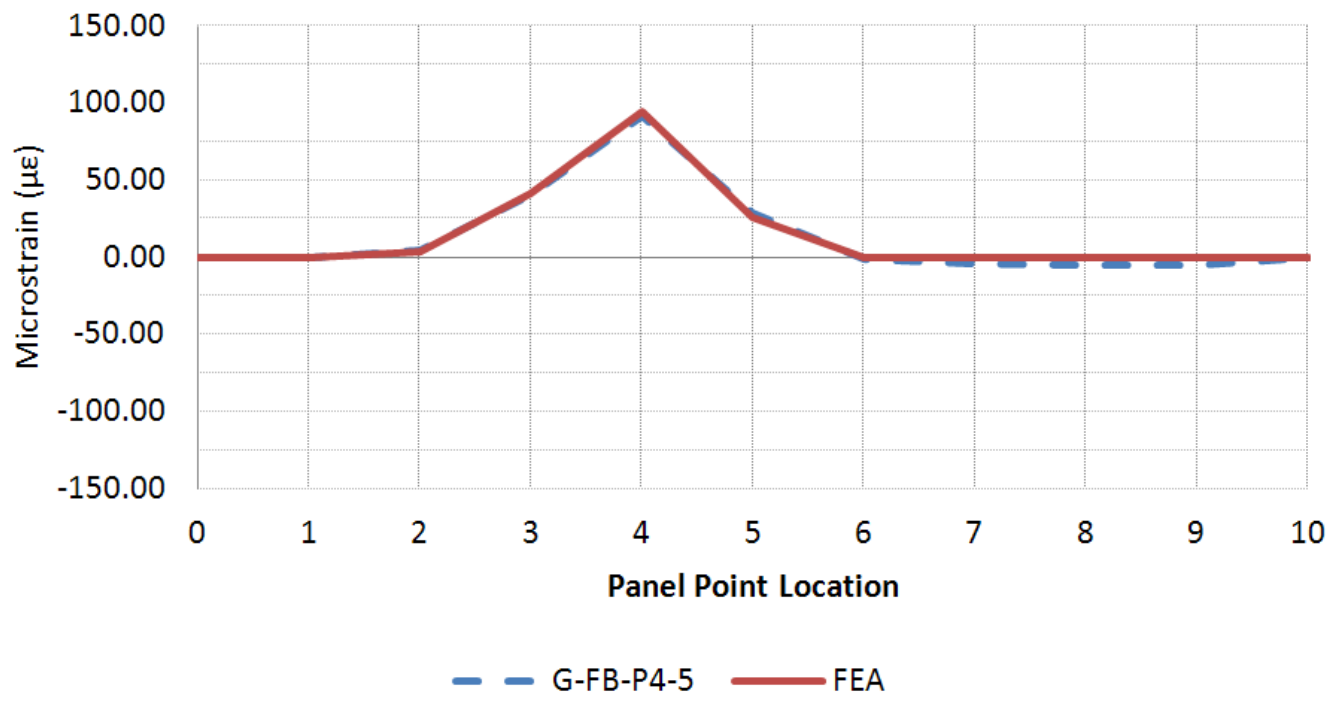

\section{A.3 Run 2 (Setup \#2, Center Truck Run)}

\section{LVDT-P3-East}

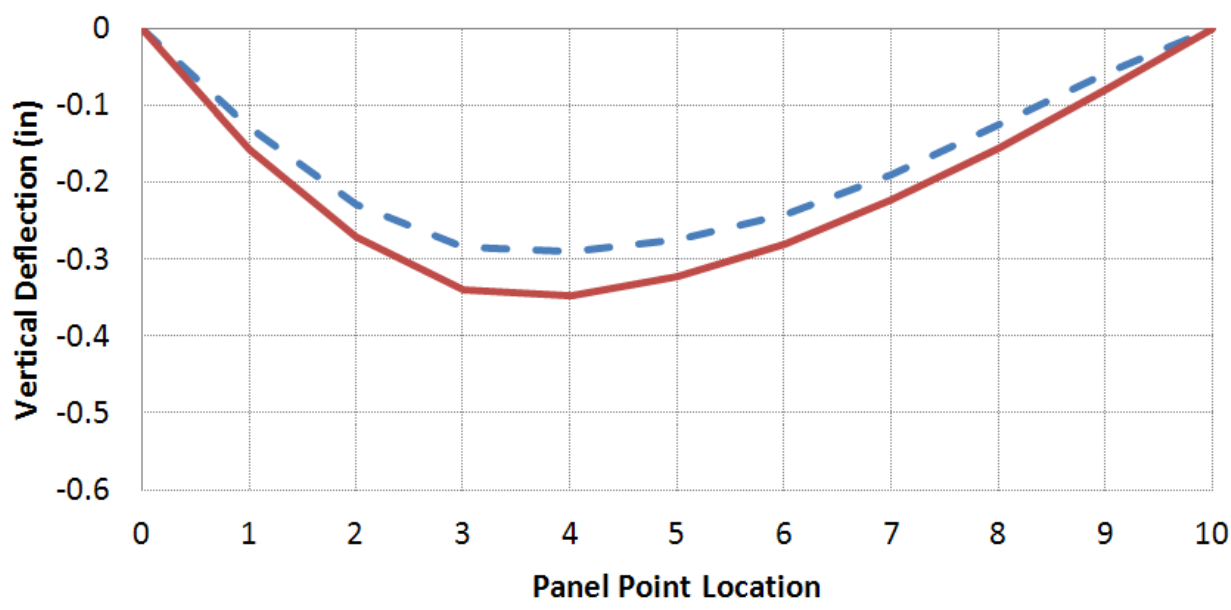

- LVDT-P3-East FEA 


\section{LVDT-P3-FB}

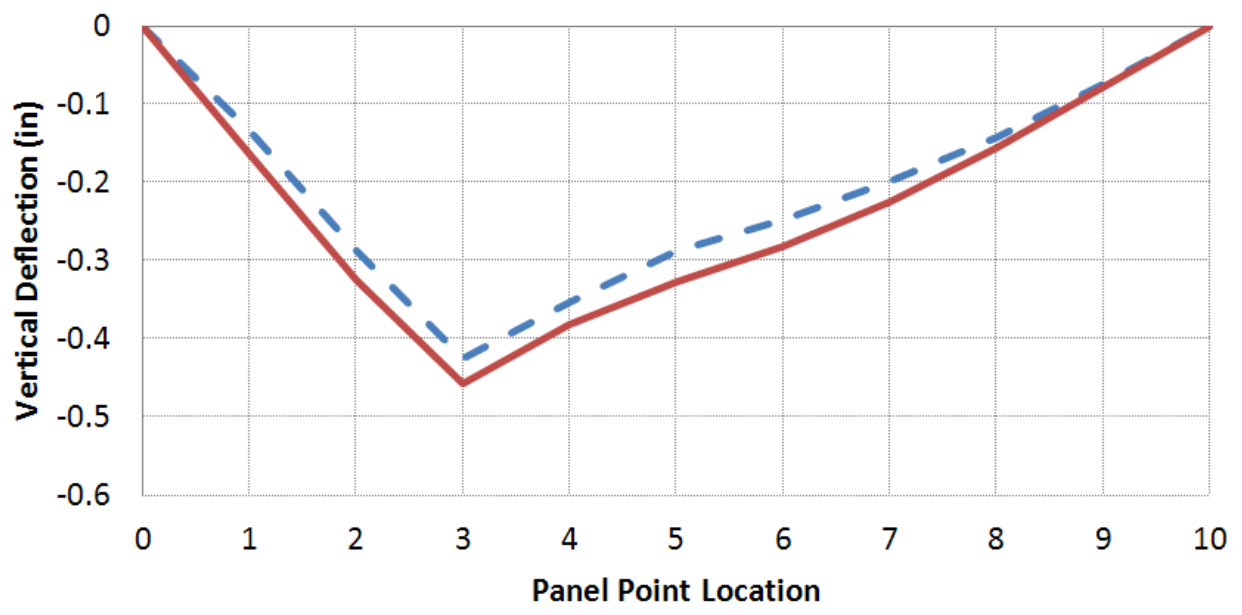

- LVDT-P3-FB FEA

\section{LVDT-P3-West}

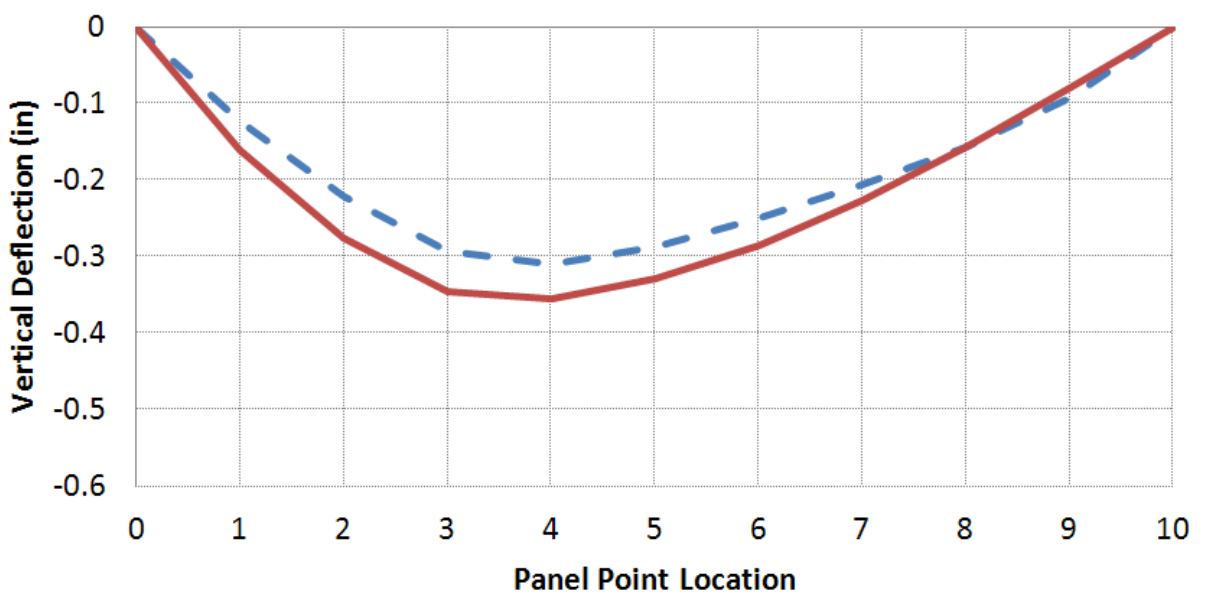

- - LVDT-P3-West FEA 


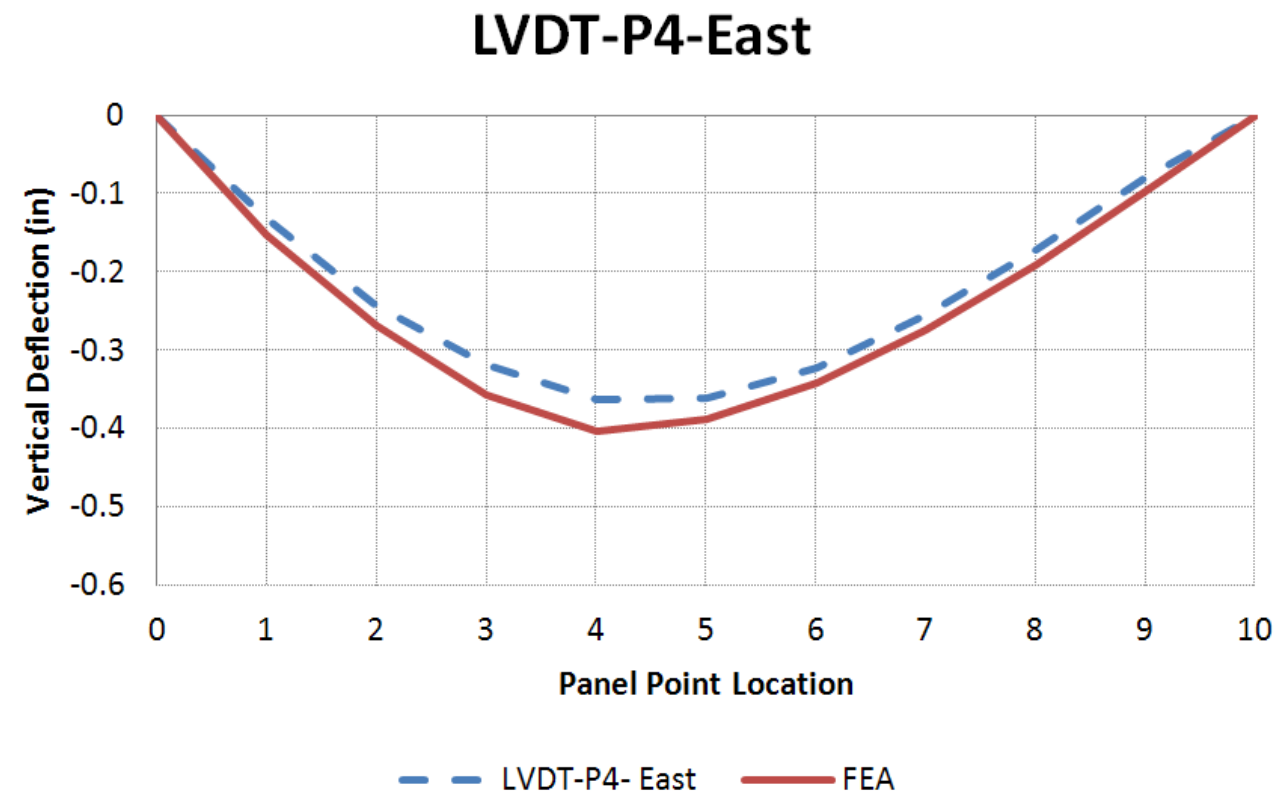

\section{LVDT-P4-FB}

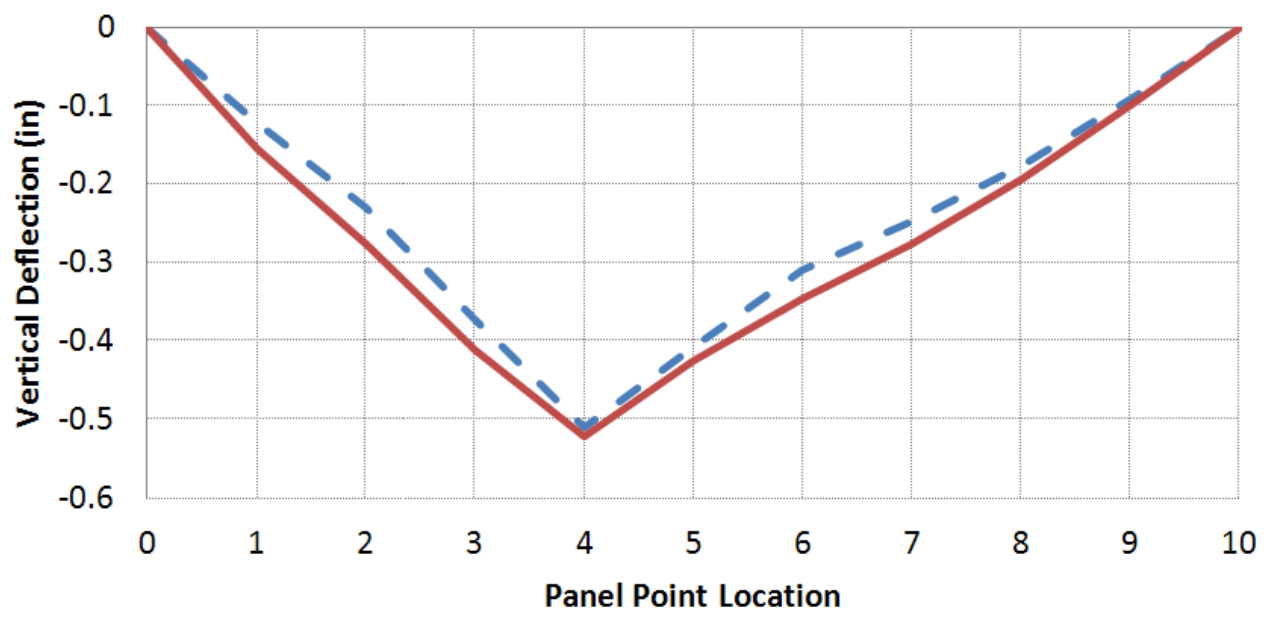

- LVDT-P4-FB FEA 


\section{LVDT-P4-West}

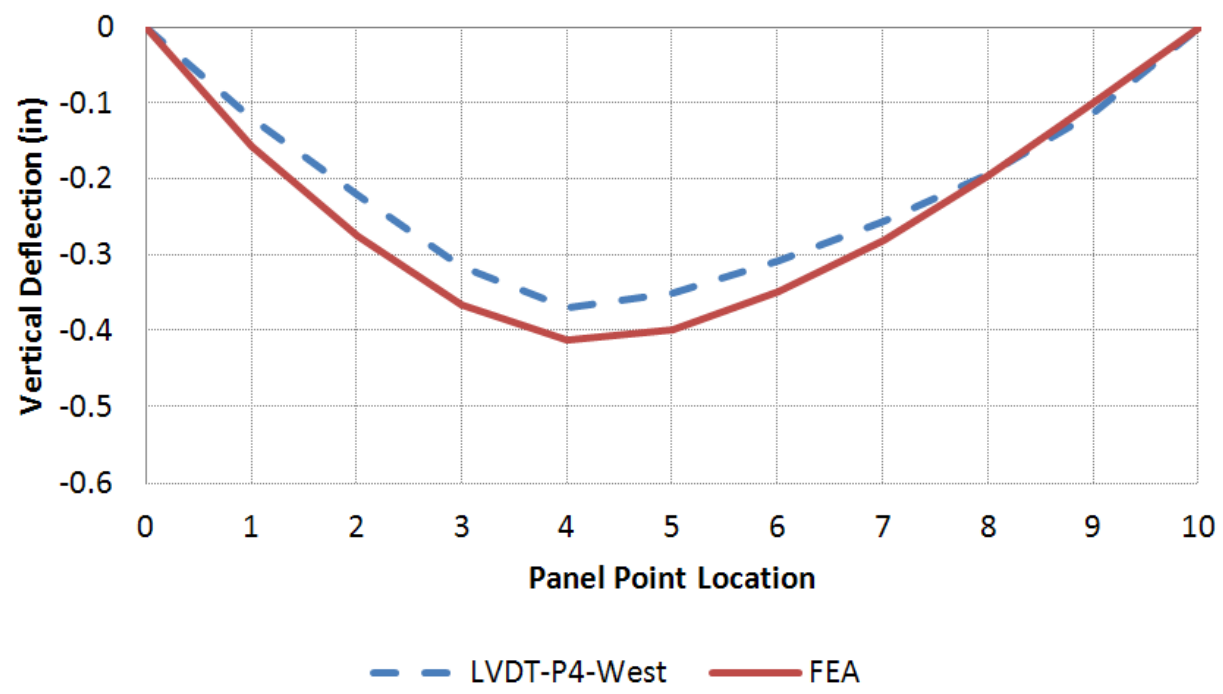

\section{G-BC-1}

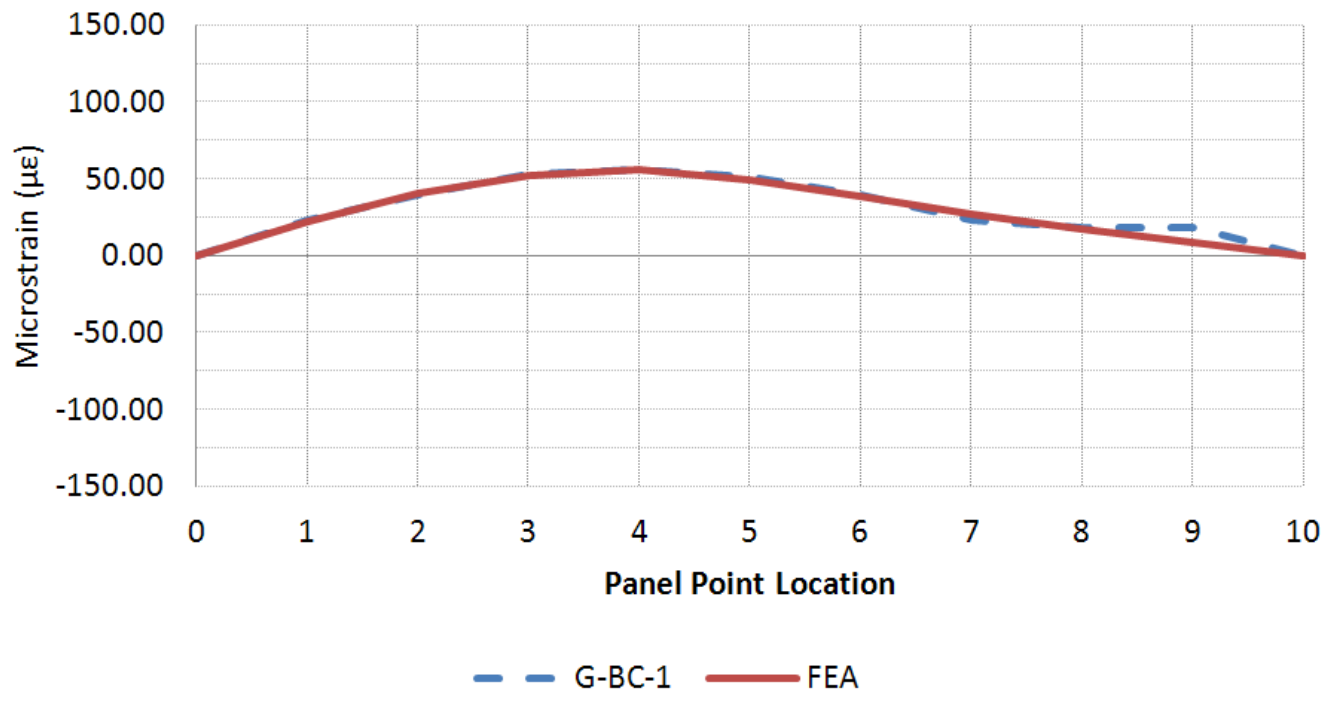




\section{G-BC-2}
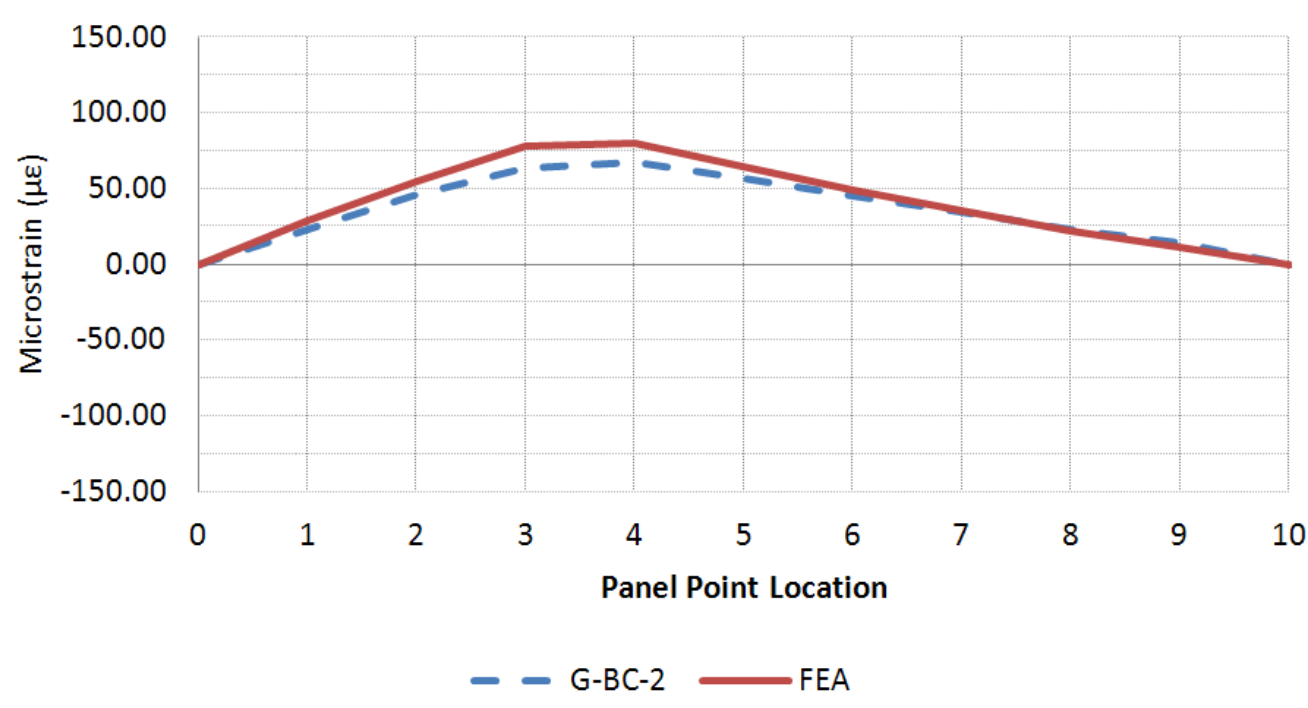

\section{G-BC-4}

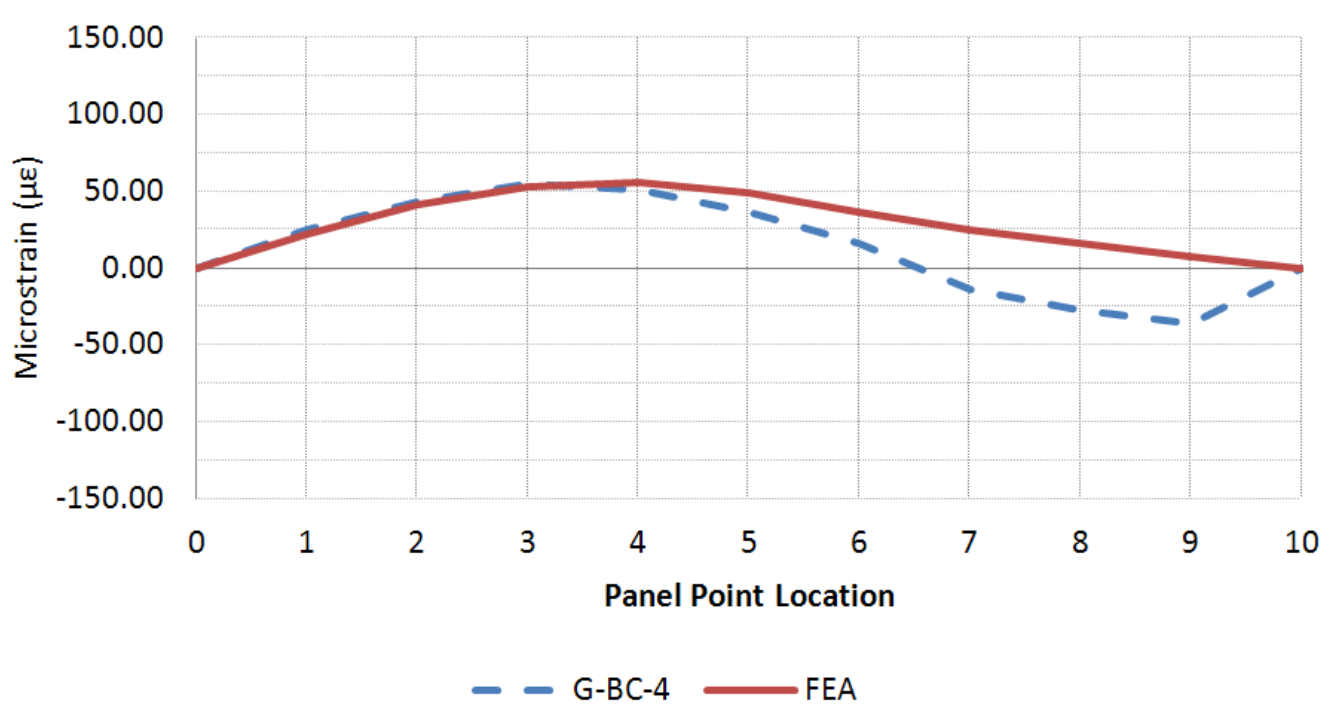




\section{G-BC-6}

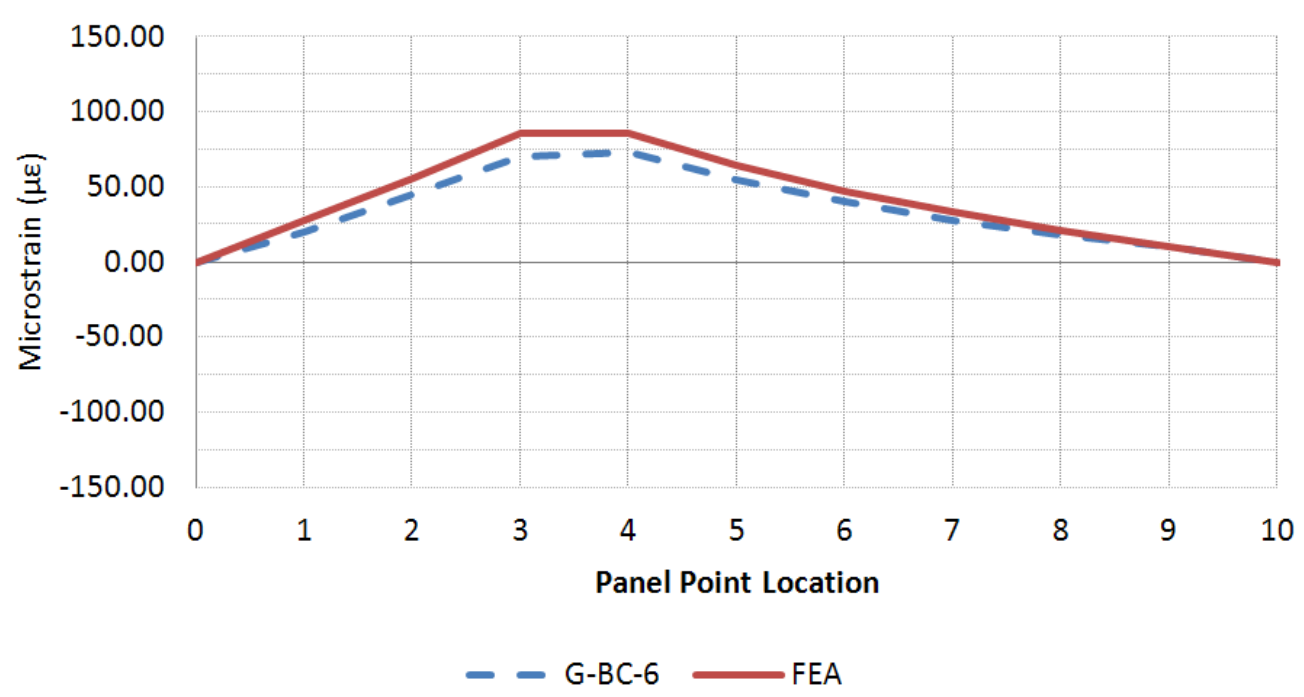

G-BC-8

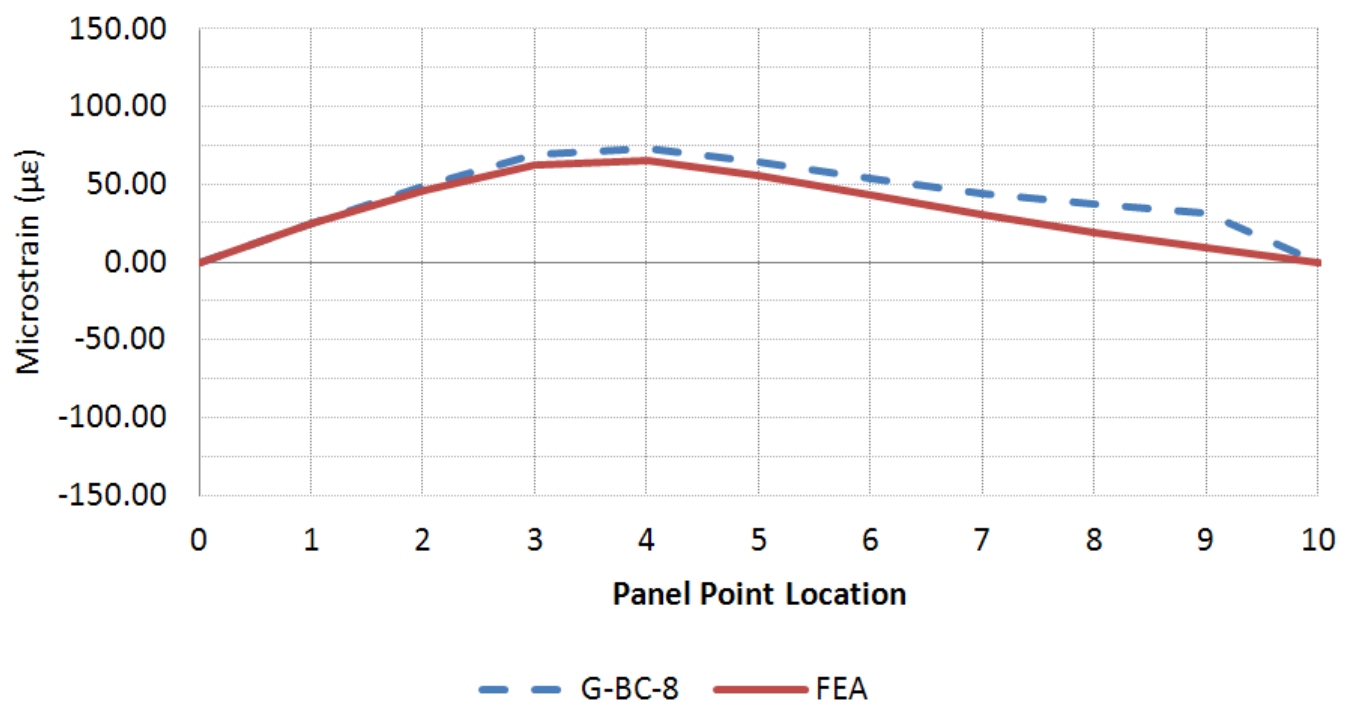




\section{G-TC-1}
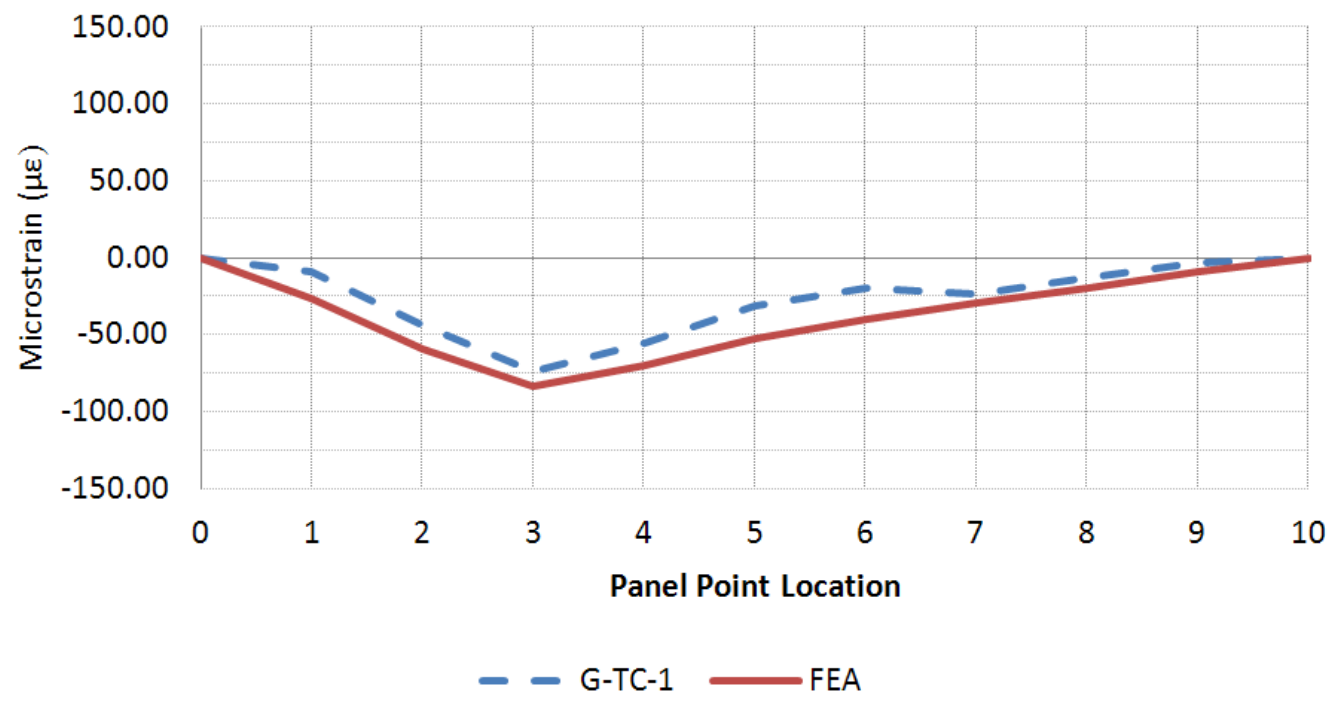

\section{G-TC-2}

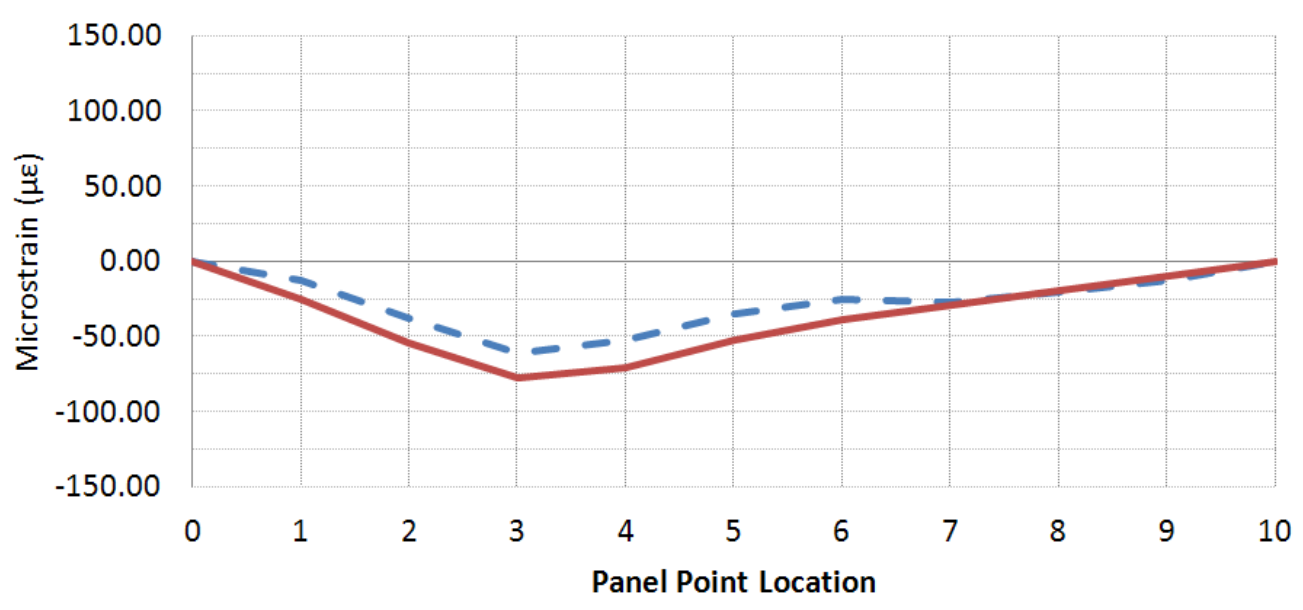

- G-TC-2 FEA 


\section{G-TC-3}

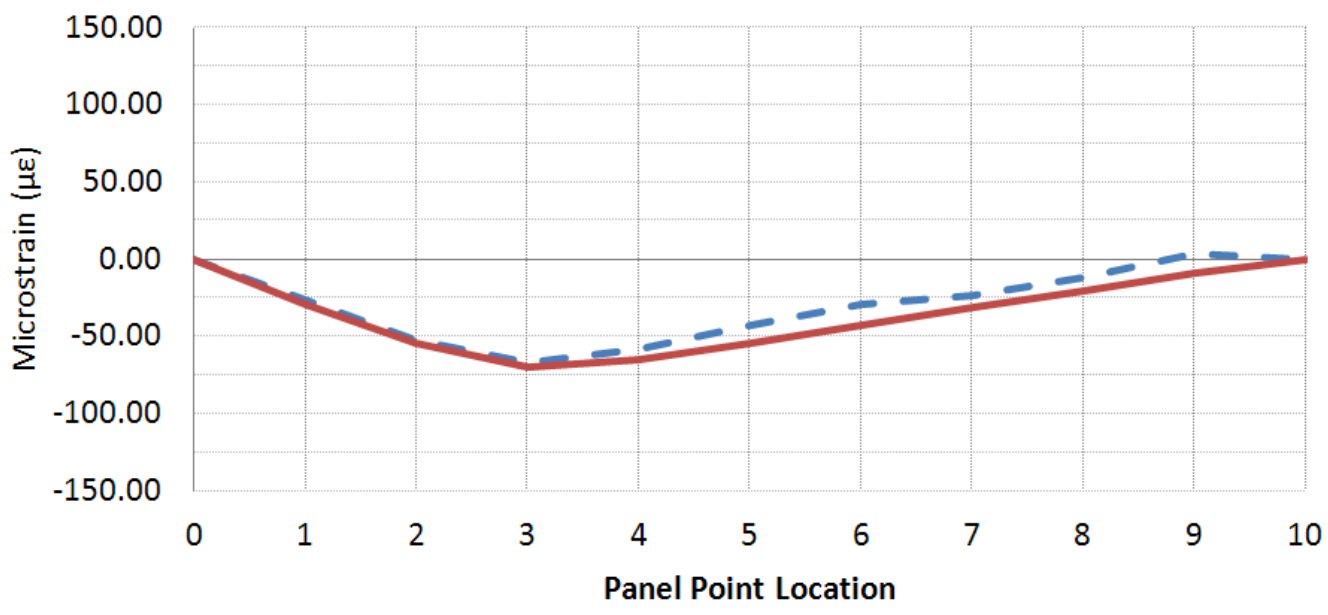

- G-TC-3 FEA

\section{G-TC-5}

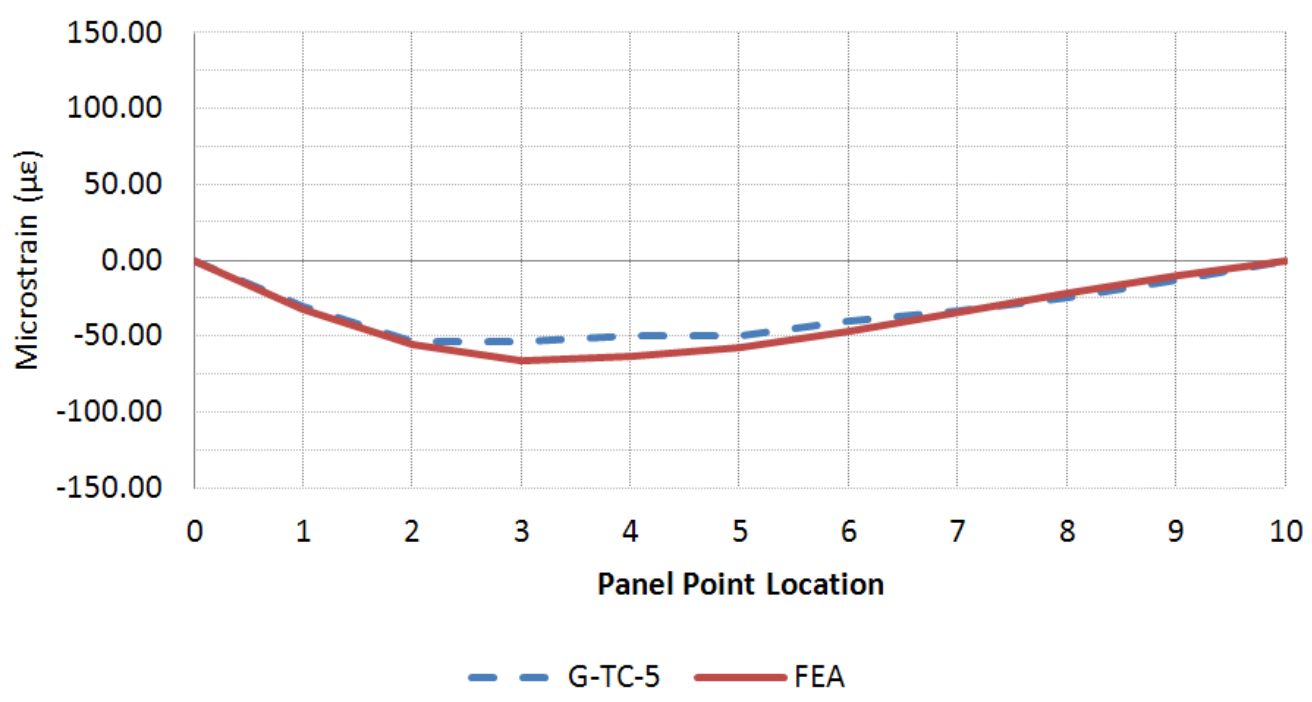




\section{G-TC-6}

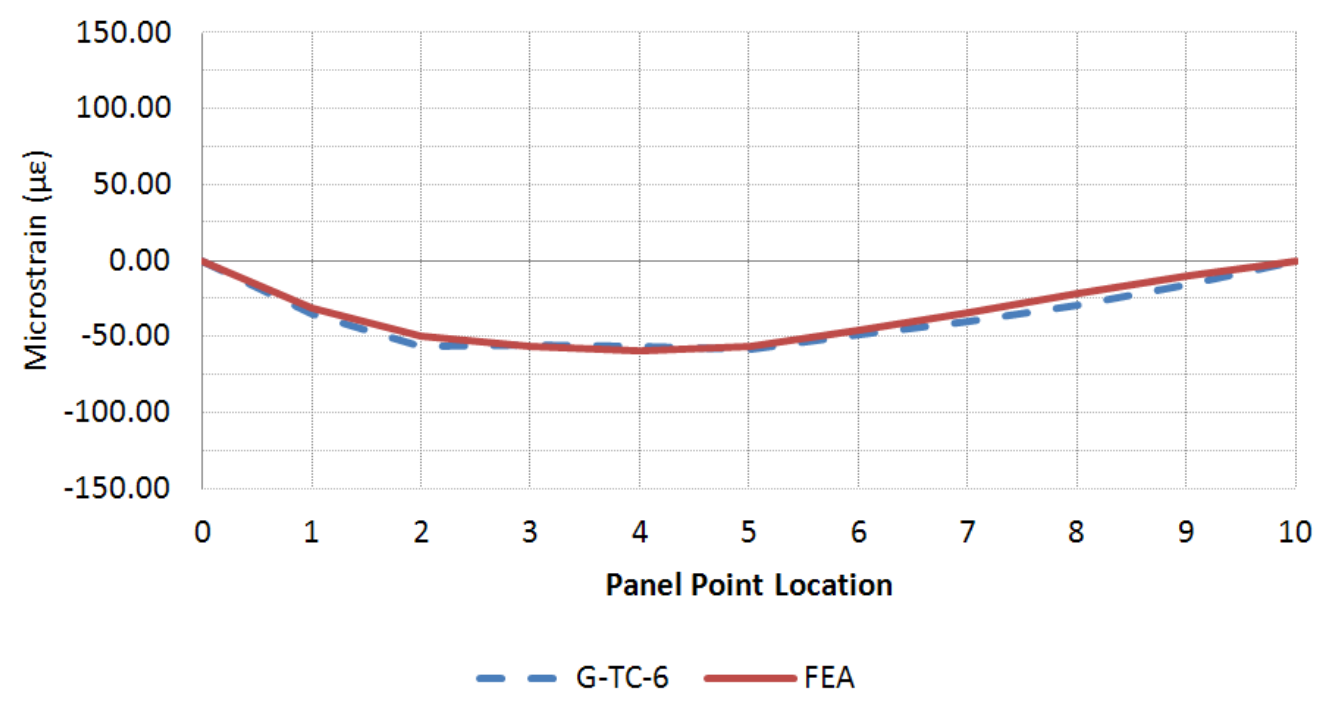

\section{A.4 Run 3 (Setup \#2, Edge Truck Run)}

\section{LVDT-P3-East}

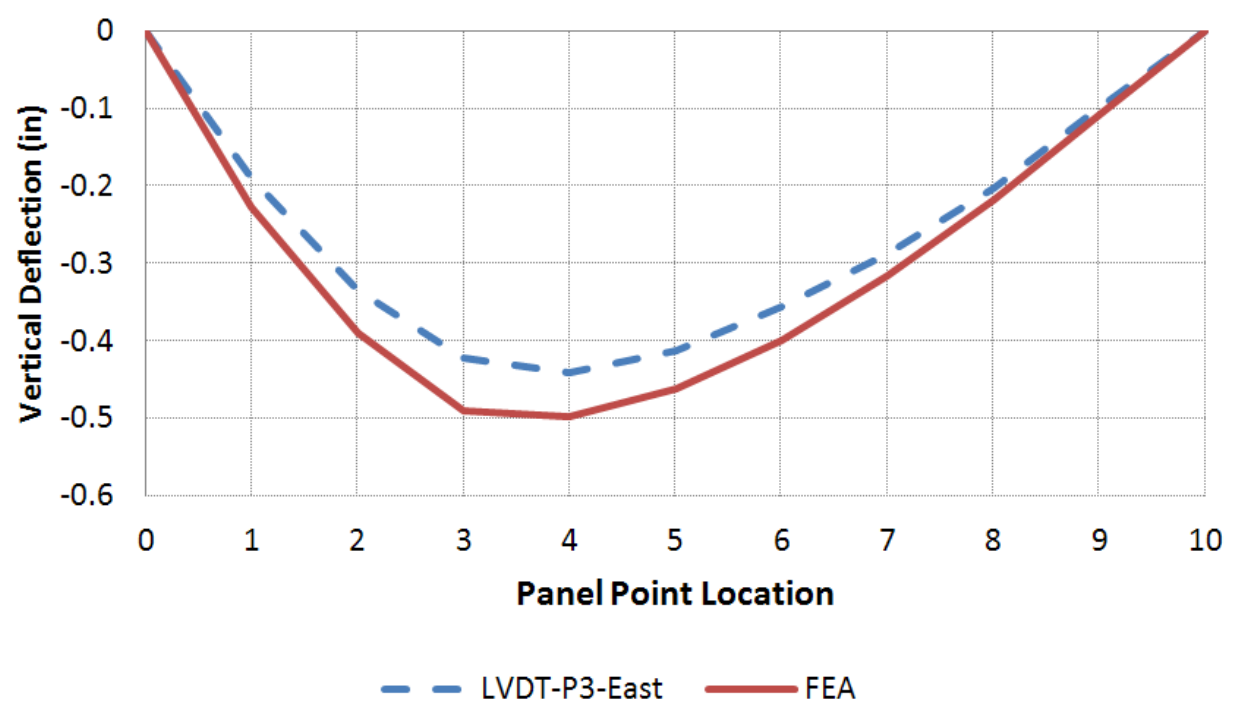




\section{LVDT-P3-FB}

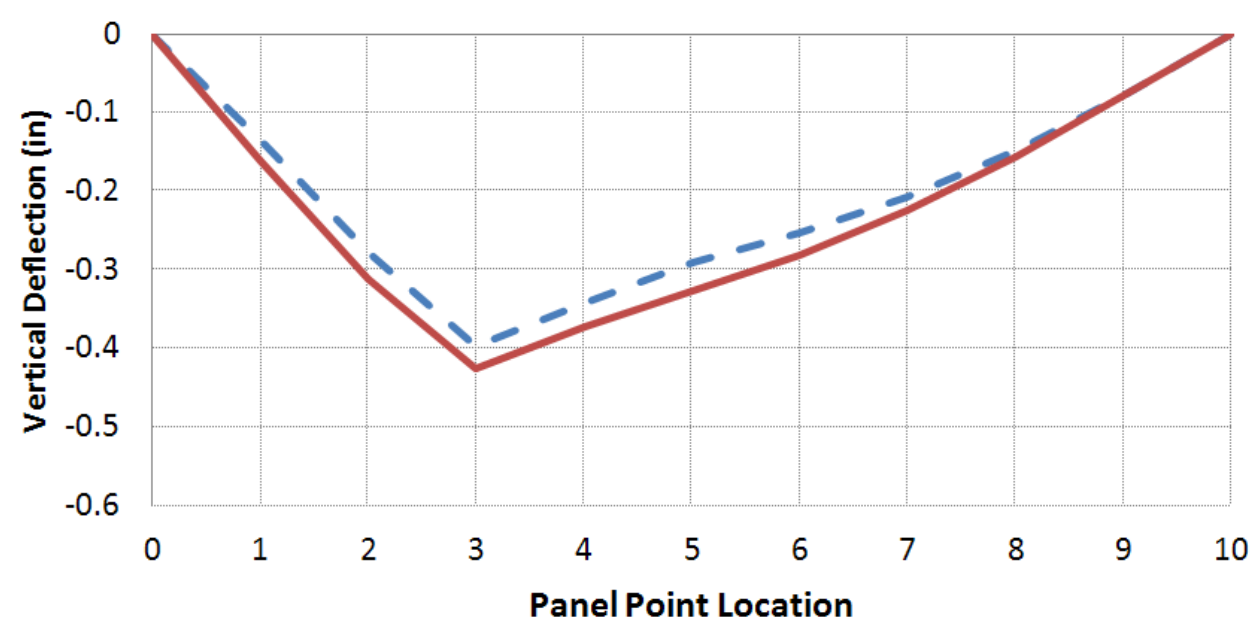

- LVDT-P3-FB FEA

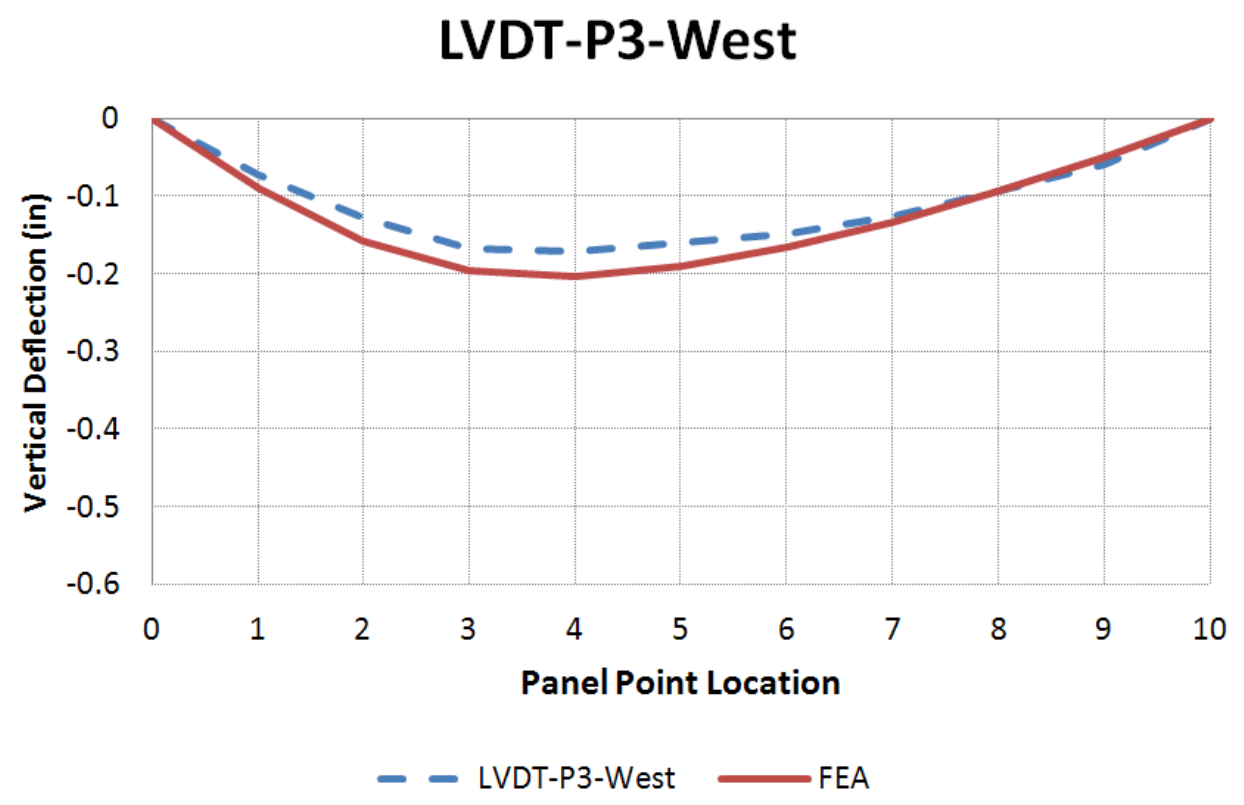




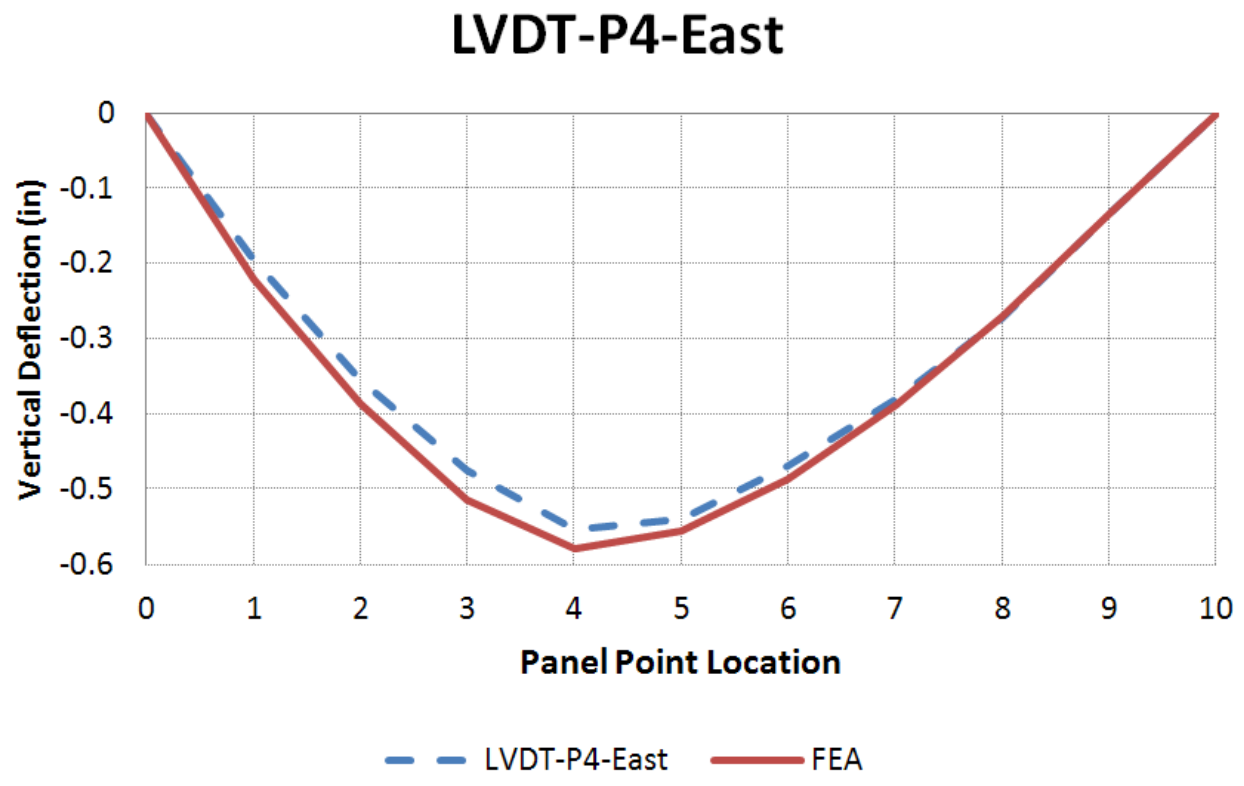

\section{LVDT-P4-FB}

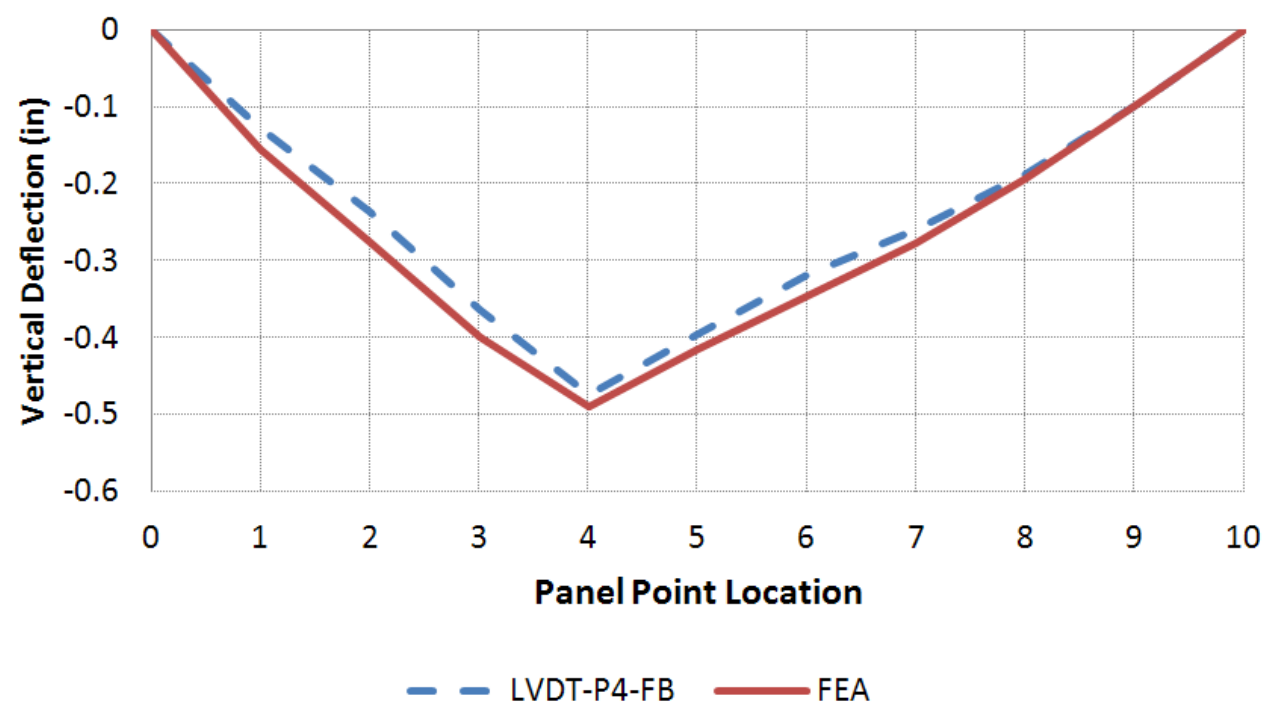




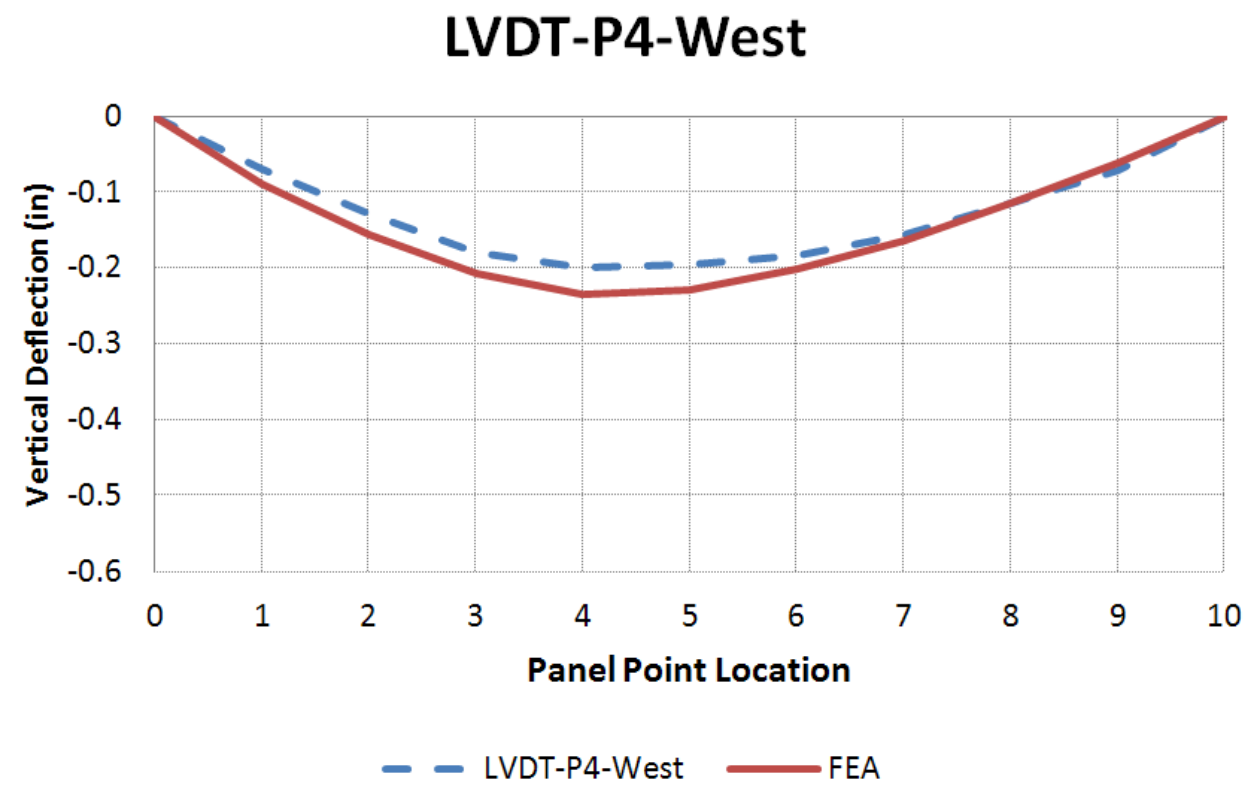

G-BC-1

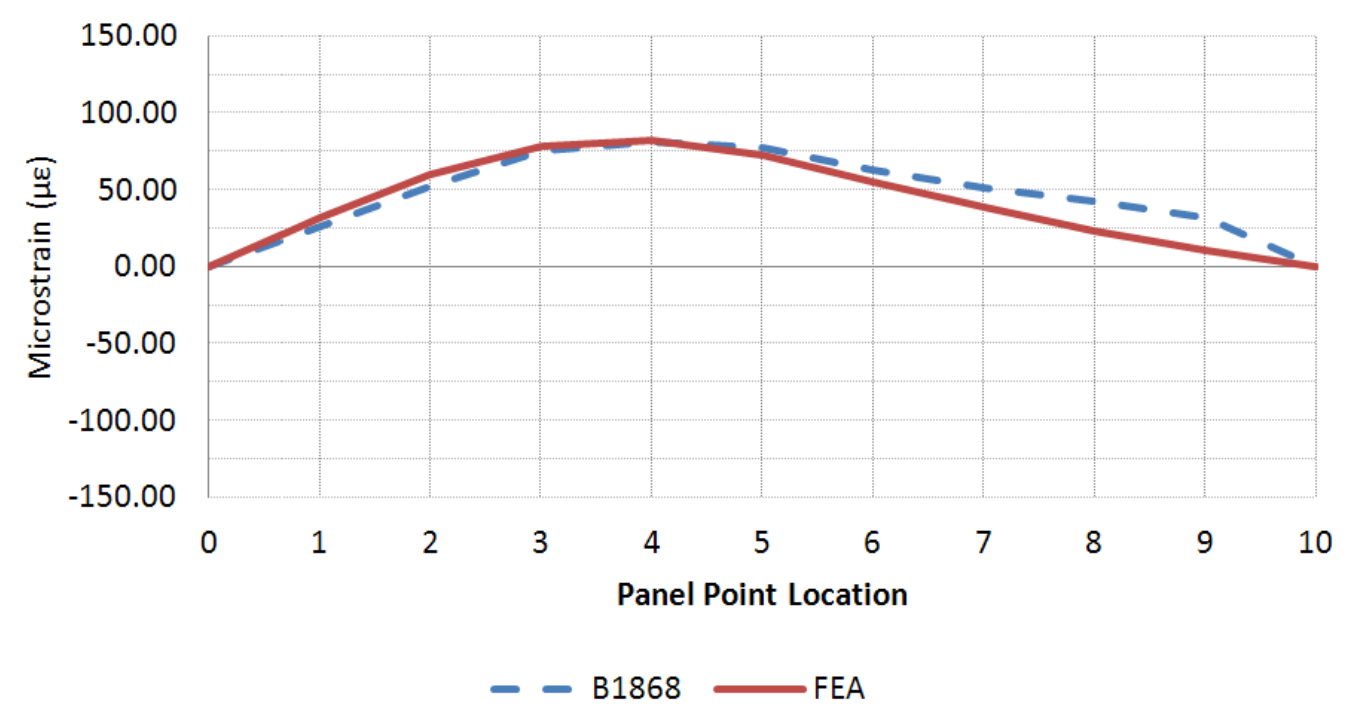


G-BC-2
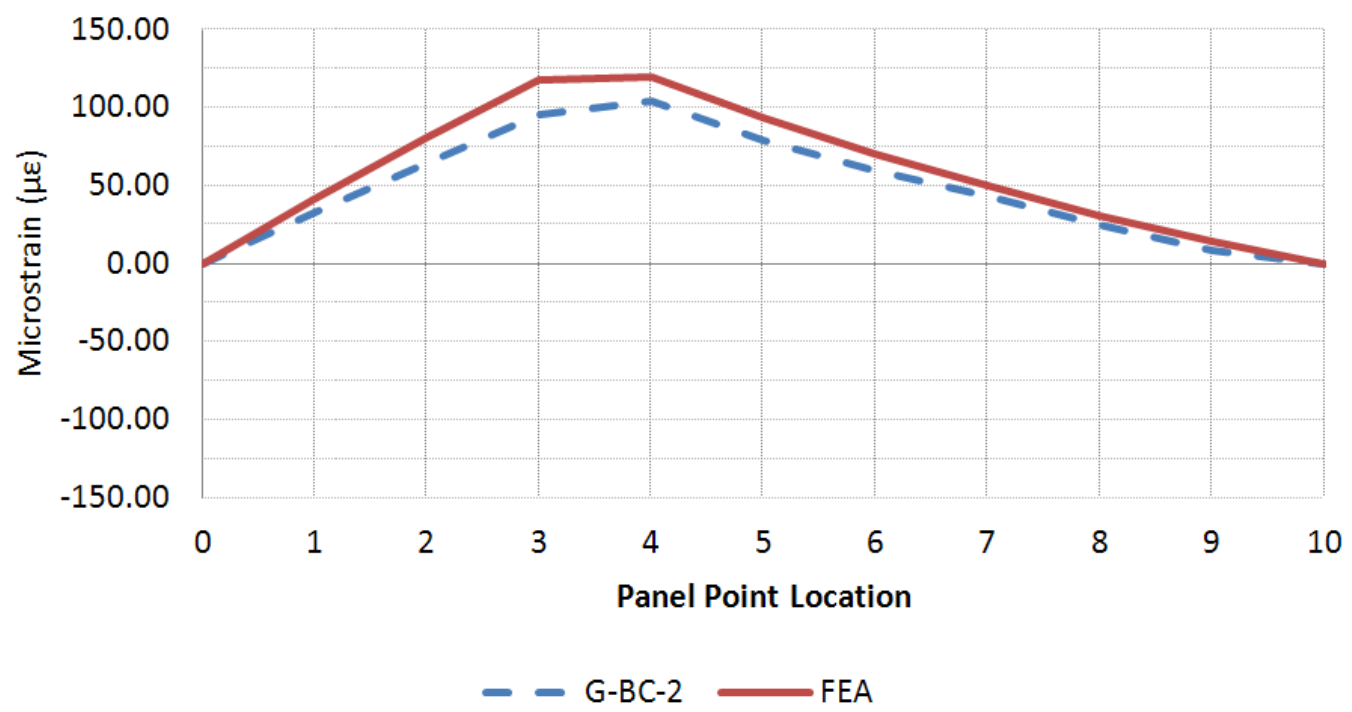

\section{G-BC-4}

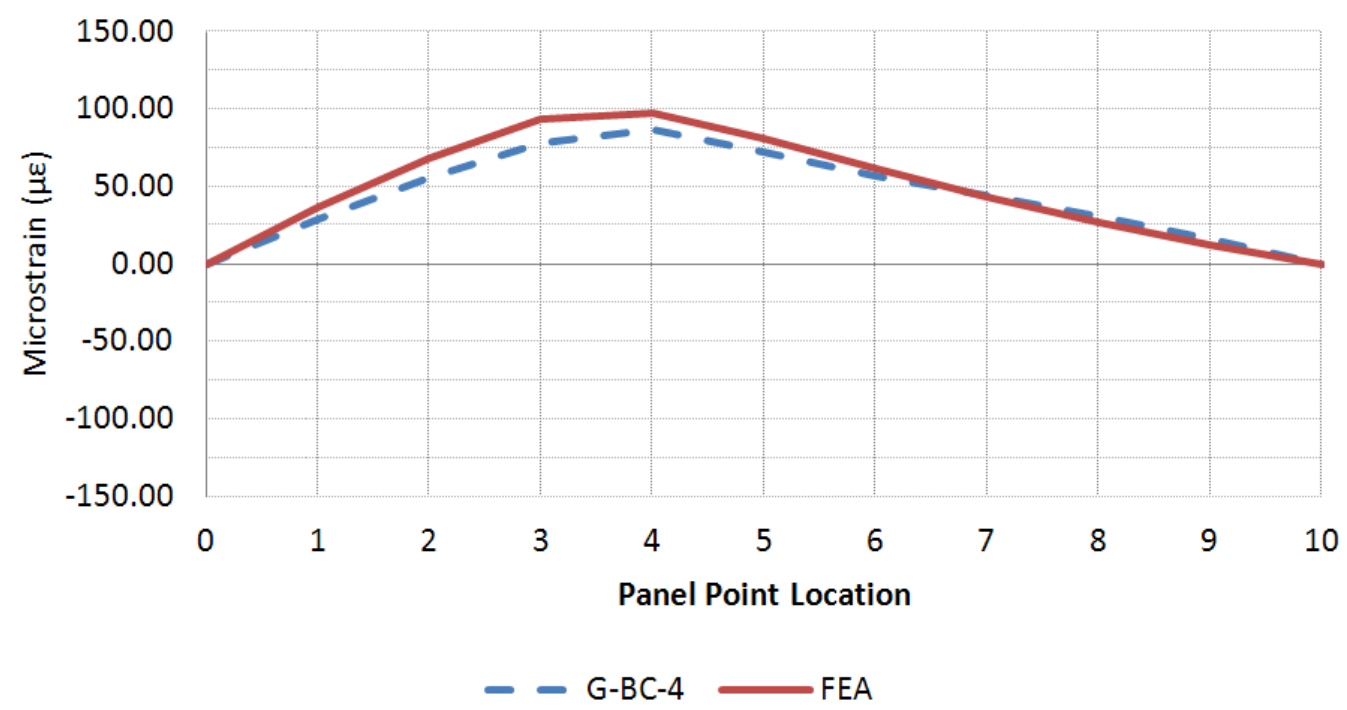




\section{G-BC-6}
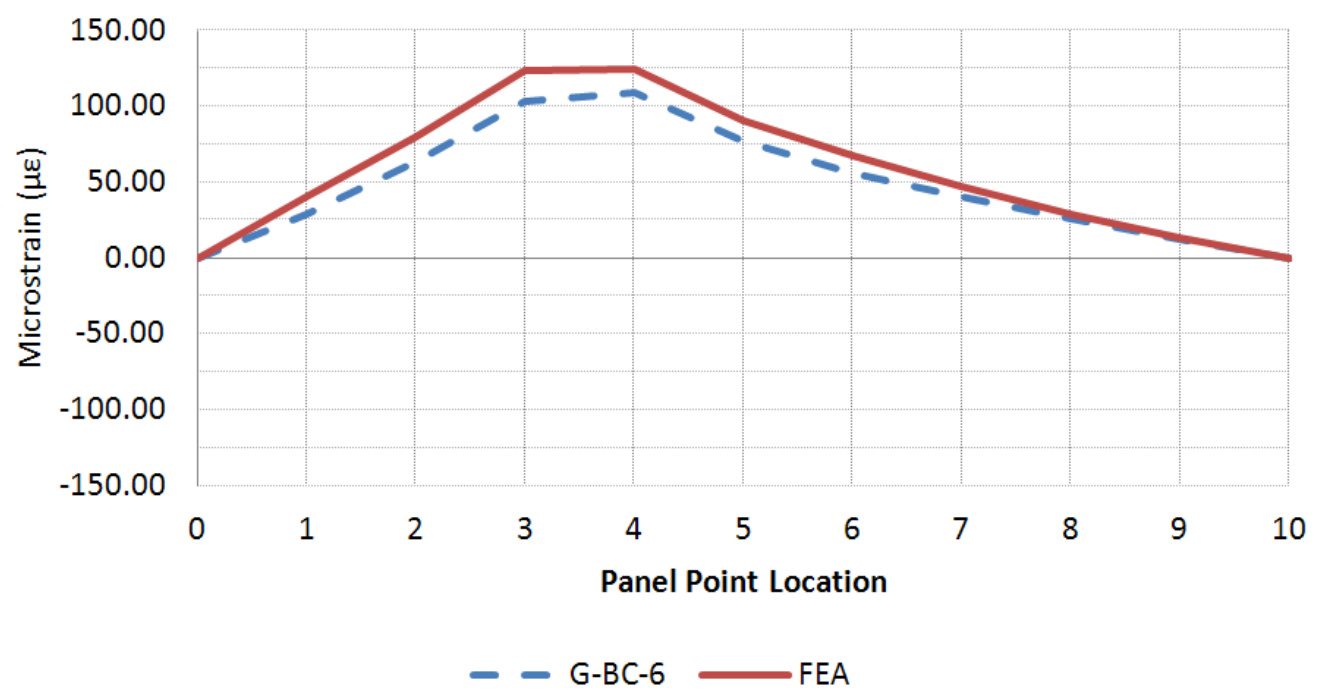

\section{G-BC-8}

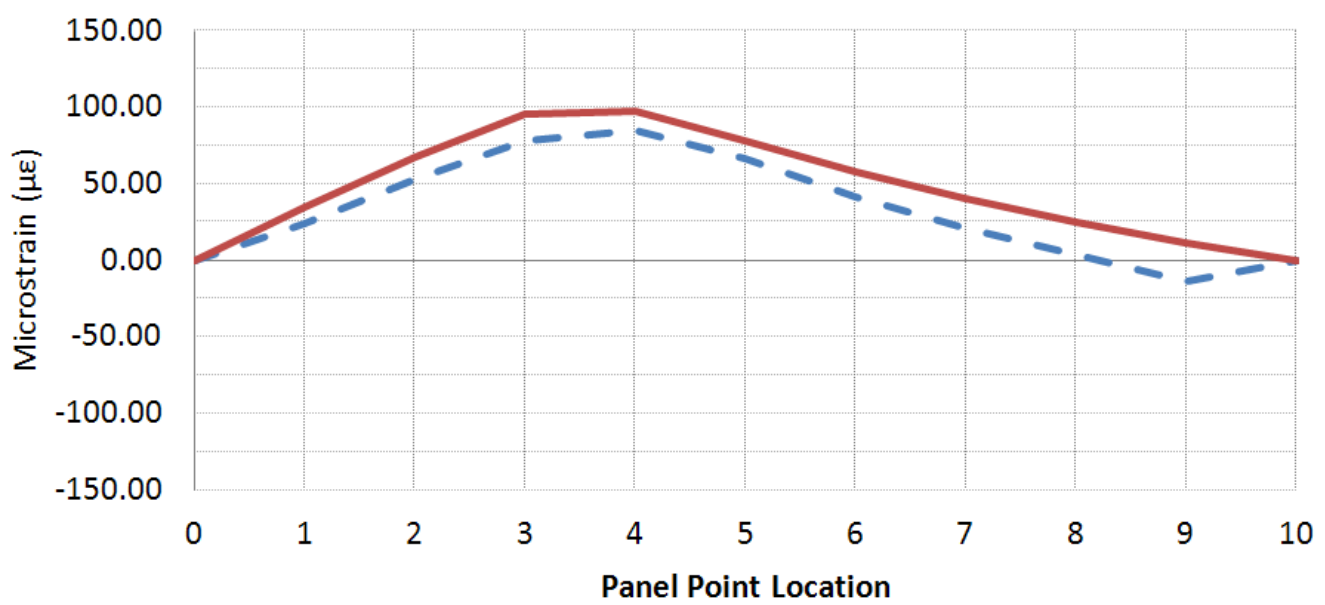

- G-BC-8 FEA 


\section{G-TC-1}
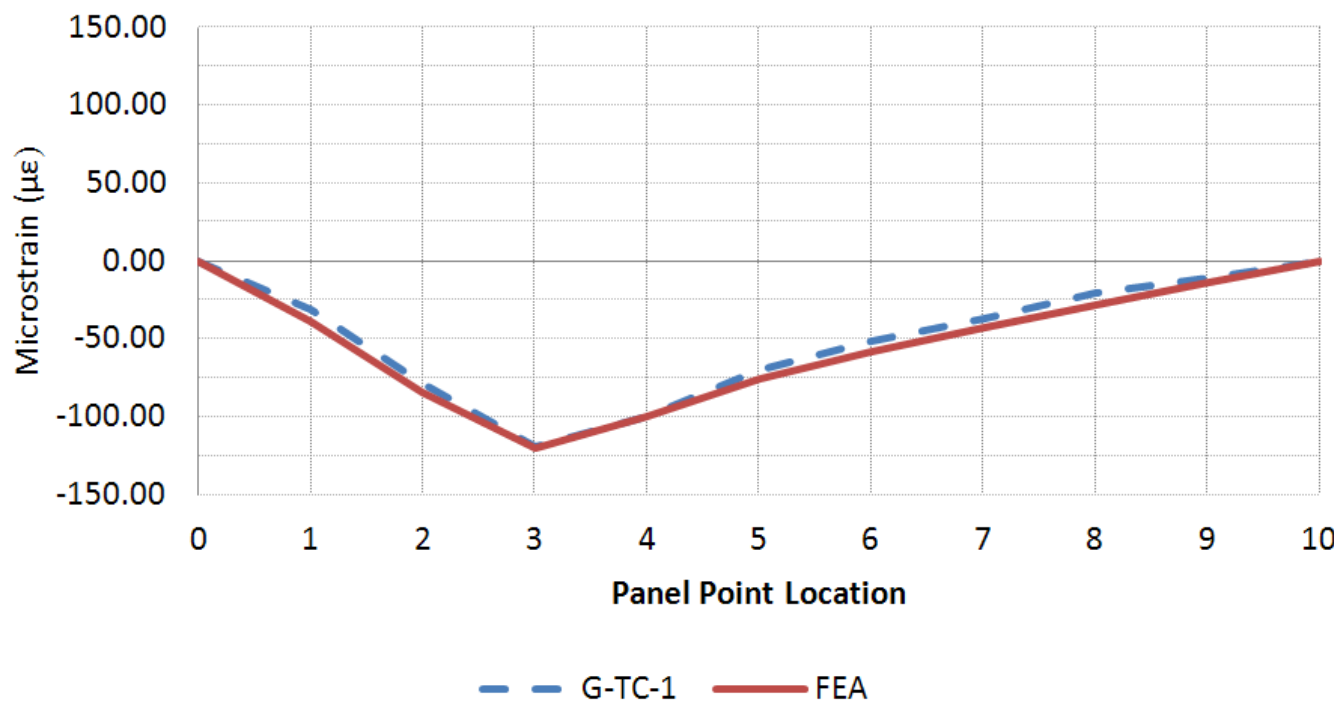

\section{G-TC-2}

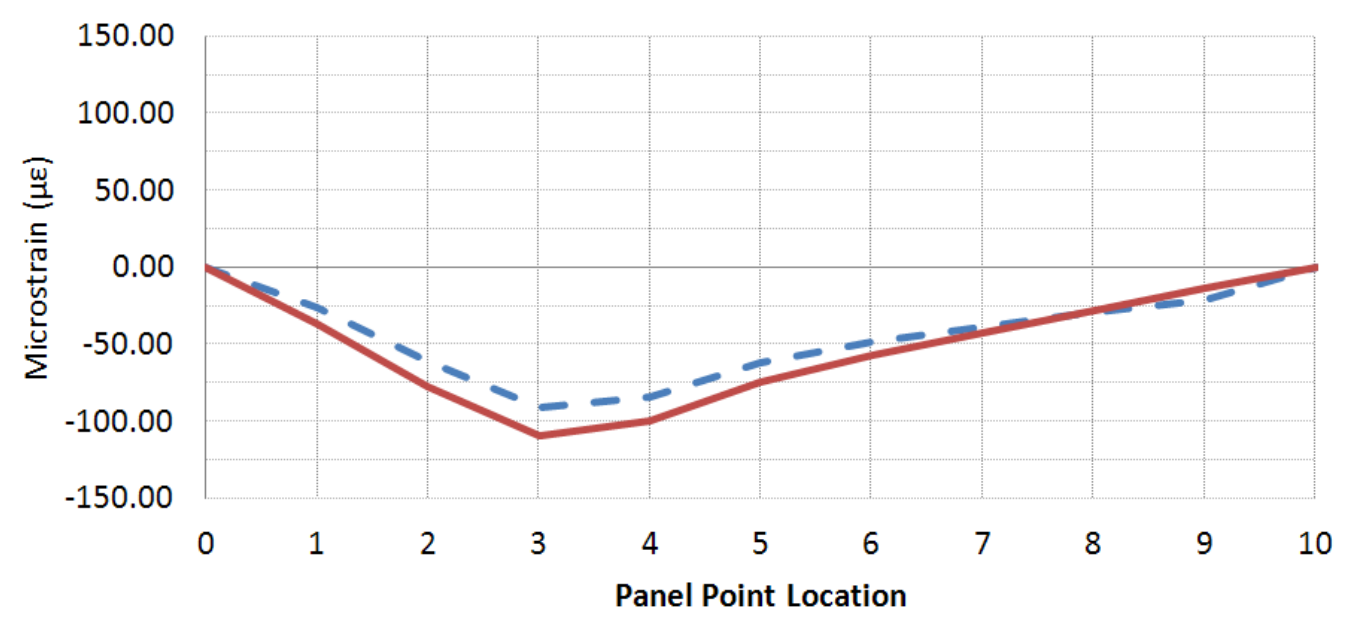

- G-TC-2 TEEA 
G-TC-3

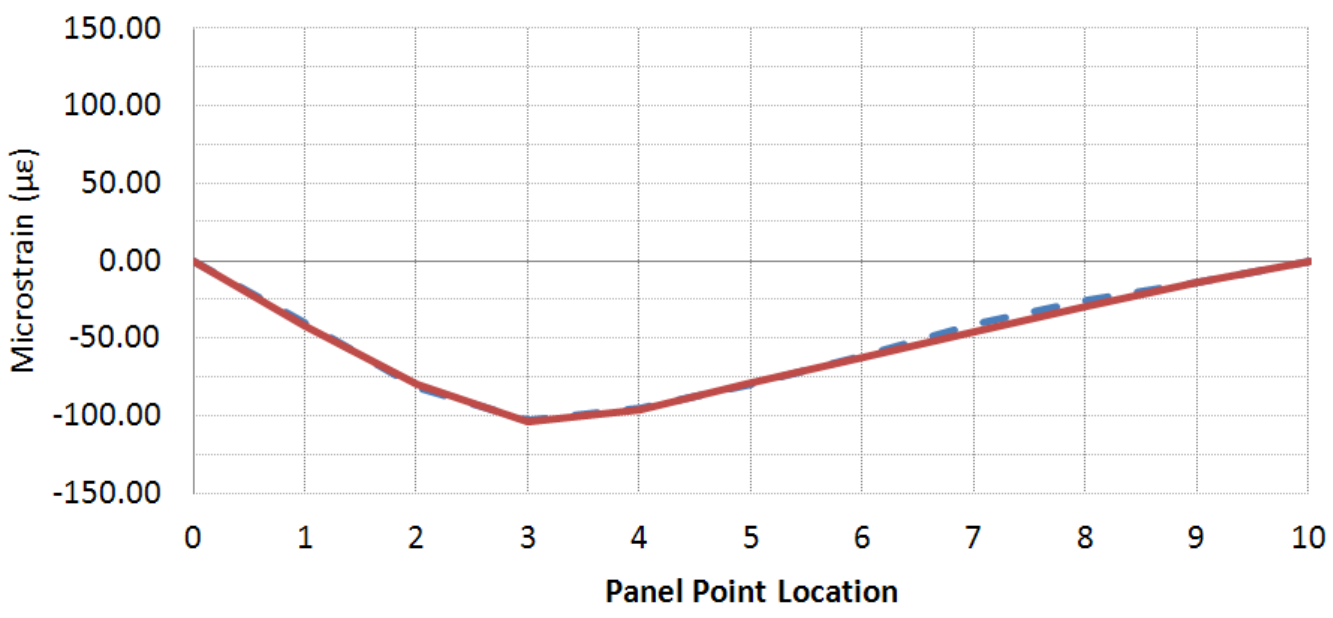

- G-TC-3 FEA

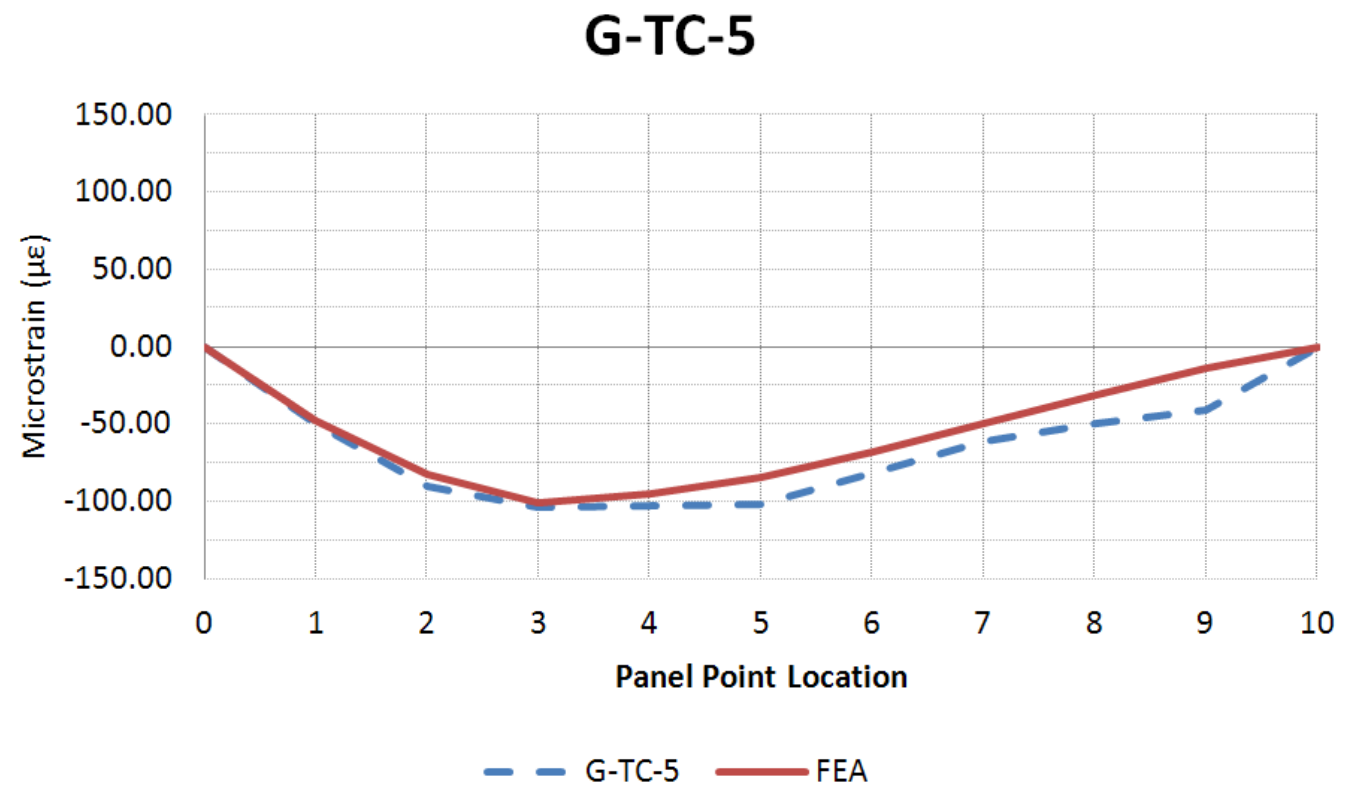




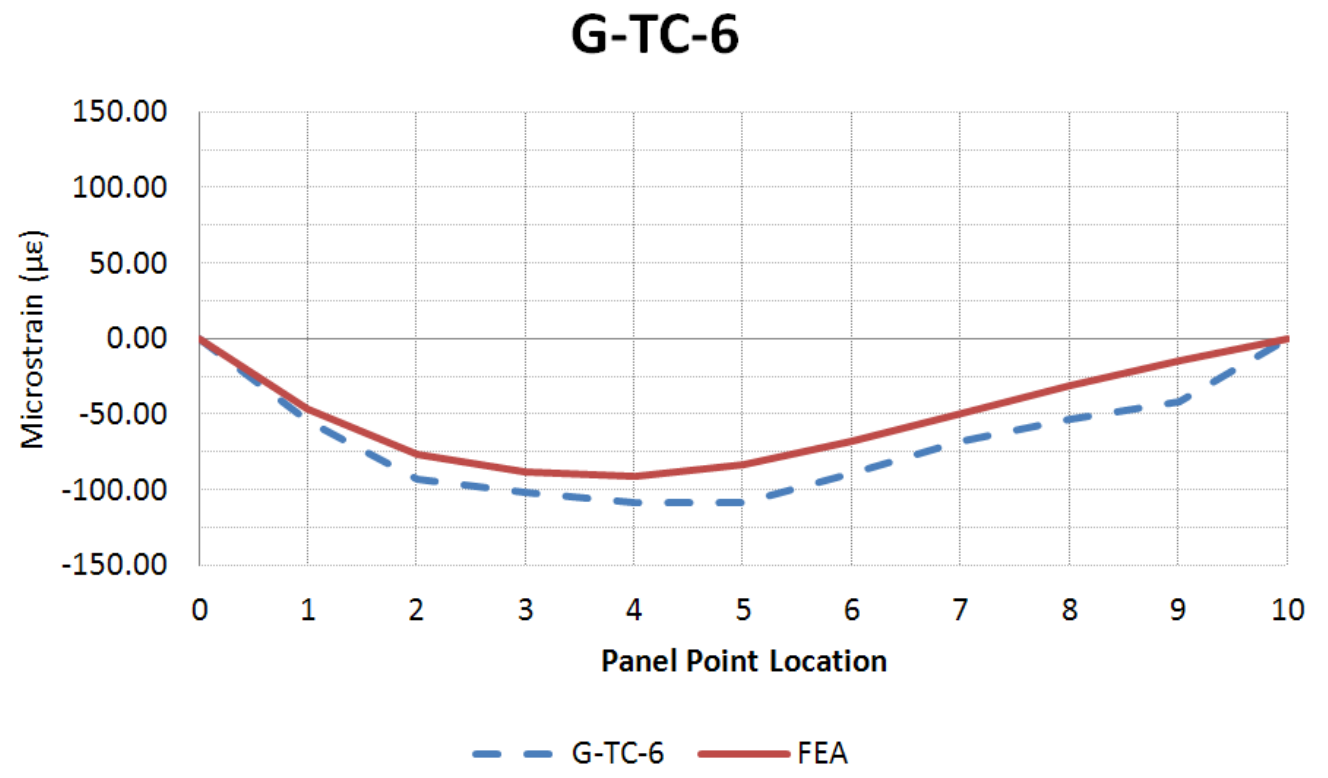

